\title{
DYNAMIC OF THRESHOLD SOLUTIONS FOR ENERGY-CRITICAL NLS
}

\author{
THOMAS DUYCKAERTS ${ }^{1}$ AND FRANK MERLE ${ }^{2}$
}

\begin{abstract}
We consider the energy-critical non-linear focusing Schrödinger equation in dimension $N=3,4,5$. An explicit stationnary solution, $W$, of this equation is known. In KM06, the energy $E(W)$ has been shown to be a threshold for the dynamical behavior of solutions of the equation. In the present article, we study the dynamics at the critical level $E(u)=E(W)$ and classify the corresponding solutions. This gives in particular a dynamical characterization of $W$.
\end{abstract}

\section{Contents}

1. Introduction

2. Compactness properties for nonlinear subcritical threshold solutions

3. Convergence to $W$ in the subcritical case

4. Convergence to $W$ in the supercritical case

5. Preliminaries on the linearized equation around $W$

6. Proof of main results

7. Appendix

References

\section{INTRODUCTION}

We consider the focusing energy-critical Schrödinger equation on an interval $I(0 \in I)$

$$
\left\{\begin{array}{c}
i \partial_{t} u+\Delta u+|u|^{p_{c}-1} u=0, \quad(t, x) \in I \times \mathbb{R}^{N} \\
u_{\uparrow t=0}=u_{0} \in \dot{H}^{1},
\end{array}\right.
$$

where

$$
N \in\{3,4,5\}, \quad p_{c}:=\frac{N+2}{N-2}
$$

and $\dot{H}^{1}:=\dot{H}^{1}\left(\mathbb{R}^{N}\right)$ is the homogeneous Sobolev space on $\mathbb{R}^{N}$ with the norm $\|f\|_{\dot{H}^{1}}^{2}:=\int|\nabla f|^{2}$. The Cauchy problem for (1.1) was studied in CW90. Namely, if $u_{0}$ is in $\dot{H}^{1}$, there exists an unique solution defined on a maximal interval $I=\left(-T_{-}, T_{+}\right)$, such that

$$
J \Subset I \Longrightarrow\|u\|_{S(J)}<\infty, \quad S(J):=L^{2 p_{c}}\left(J \times \mathbb{R}^{N}\right),
$$

Date: August 15, 2018.

${ }^{1}$ Cergy-Pontoise (UMR 8088).

${ }^{2}$ Cergy-Pontoise, IHES, CNRS.

This work was partially supported by the French ANR Grant ONDNONLIN. 
and the energy

$$
E(u(t))=\frac{1}{2} \int|\nabla u(t, x)|^{2} d x-\frac{1}{2^{*}} \int|u(t, x)|^{2^{*}} d x
$$

is constant (here $2^{*}:=\frac{2 N}{N-2}=p_{c}+1$ is the critical exponent for the $H^{1}$-Sobolev embedding in $\mathbb{R}^{N}$ ). In addition, $u$ satisfies the following global existence criterium:

$$
T_{+}<\infty \Longrightarrow\|u\|_{S\left(0, T_{+}\right)}=\infty .
$$

Moreover, solutions of equation (1.1) are invariant by the following transformations: if $u(t, x)$ is such a solution so is

$$
\frac{e^{i \theta_{0}}}{\lambda_{0}^{(N-2) / 2}} u\left(\frac{t_{0}+t}{\lambda_{0}^{2}}, \frac{x_{0}+x}{\lambda_{0}}\right), \quad\left(\theta_{0}, \lambda_{0}, t_{0}, x_{0}\right) \in \mathbb{R} \times(0, \infty) \times \mathbb{R} \times \mathbb{R}^{N} .
$$

Note that these transformations preserve the $S(\mathbb{R})$-norm, as well as the $\dot{H}^{1}$-norm, the $L^{2^{*}}$-norm and thus the energy.

An explicit solution of (1.1) is the stationnary solution in $\dot{H}^{1}$ (but in $L^{2}$ only if $N \geq 5$ )

$$
W:=\frac{1}{\left(1+\frac{|x|^{2}}{N(N-2)}\right)^{\frac{N-2}{2}}} .
$$

The works of Aubin and Talenti [Aub76, Tal76], give the following elliptic characterization of $W$

$$
\begin{gathered}
\forall u \in \dot{H}^{1}, \quad\|u\|_{L^{2^{*}}} \leq C_{N}\|u\|_{\dot{H}^{1}} \\
\|u\|_{L^{2 *}}=C_{N}\|u\|_{\dot{H}^{1}} \Longrightarrow \exists \lambda_{0}, x_{0}, z_{0} \quad u(x)=z_{0} W\left(\frac{x+x_{0}}{\lambda_{0}}\right),
\end{gathered}
$$

where $C_{N}$ is the best Sobolev constant in dimension $N$.

In [KM06, Kenig and Merle has shown that $W$ plays an important role in the dynamical behavior of solutions of Equation (1.1). Indeed, $E(W)=\frac{1}{N C_{N}^{N}}$ is an energy threshold for the dynamics in the following sense. Let $u$ be a radial solution of (1.1) such that

$$
E\left(u_{0}\right)<E(W) .
$$

Then if $\left\|u_{0}\right\|_{\dot{H}^{1}}<\|W\|_{\dot{H}^{1}}$, we have

$$
T_{+}=T_{-}=\infty \text { and }\|u\|_{S(\mathbb{R})}<\infty .
$$

On the other hand if $\left\|u_{0}\right\|_{\dot{H}^{1}}>\|W\|_{\dot{H}^{1}}$, and $u_{0} \in L^{2}$ then

$$
T_{+}<\infty \text { and } T_{-}<\infty \text {. }
$$

Our goal is to give a classification of solutions of (1.1) with critical energy, that is with initial condition such that

$$
u_{0} \in \dot{H}^{1}, \quad E\left(u_{0}\right)=E(W) .
$$

A new example of such a solution (not satisfying (1.6) nor (1.7)) is given by $W$. We start with the following theorem, which shows that the dynamics at this critical level is richer, in the sense that there exists orbit connecting different types of behavior for $t>0$ and $t<0$. 
Theorem 1. Let $N \in\{3,4,5\}$. There exist radial solutions $W^{-}$and $W^{+}$of (1.1) such that

$$
\begin{gathered}
E(W)=E\left(W^{+}\right)=E\left(W^{-}\right), \\
T_{+}\left(W^{-}\right)=T_{+}\left(W^{+}\right)=+\infty \text { and } \lim _{t \rightarrow+\infty} W^{ \pm}(t)=W \text { in } \dot{H}^{1}, \\
\left\|W^{-}\right\|_{\dot{H}^{1}}<\|W\|_{\dot{H}^{1}}, \quad T_{-}\left(W^{-}\right)=+\infty, \quad\left\|W^{-}\right\|_{S((-\infty, 0])}<\infty, \\
\left\|W^{+}\right\|_{\dot{H}^{1}}>\|W\|_{\dot{H}^{1}}, \text { and, if } N=5, T_{-}\left(W^{+}\right)<+\infty .
\end{gathered}
$$

Remark 1.1. As for $W, W^{+}(t)$ and $W^{-}(t)$ belongs to $L^{2}$ if and only if $N=5$. We still expect $T_{-}\left(W^{+}\right)<+\infty$ for $N=3,4$.

Our classification result is as follows.

Theorem 2. Let $N \in\{3,4,5\}$. Let $u_{0} \in \dot{H}^{1}$ radial, such that

$$
E\left(u_{0}\right)=E(W)=\frac{1}{N C_{N}^{N}} .
$$

Let $u$ be the solution of (1.1) with initial condition $u_{0}$ and $I$ its maximal interval of definition. Then the following holds:

(a) If $\int\left|\nabla u_{0}\right|^{2}<\int|\nabla W|^{2}=\frac{1}{C_{N}^{N}}$ then $I=\mathbb{R}$. Furthermore, either $u=W^{-}$up to the symmetry of the equation, or $\|u\|_{S(\mathbb{R})}<\infty$.

(b) If $\int\left|\nabla u_{0}\right|^{2}=\int|\nabla W|^{2}$ then $u=W$ up to the symmetry of the equation.

(c) If $\int\left|\nabla u_{0}\right|^{2}>\int|\nabla W|^{2}$, and $u_{0} \in L^{2}$ then either $u=W^{+}$up to the symmetry of the equation, or I is finite.

The constant $C_{N}$ is defined in (1.3). In the theorem, by $u$ equals $v$ up to the $\left(\dot{H}^{1}-\right)$ symmetry of the equation, we mean that there exist $t_{0} \in \mathbb{R}, \theta_{0} \in \mathbb{R}, \lambda_{0}>0$ such that

$$
u(t, x)=\frac{e^{i \theta_{0}}}{\lambda_{0}^{(N-2) / 2}} v\left(\frac{t_{0}+t}{\lambda_{0}^{2}}, \frac{x}{\lambda_{0}}\right) \text { or } u(t, x)=\frac{e^{i \theta_{0}}}{\lambda_{0}^{(N-2) / 2}} \bar{v}\left(\frac{t_{0}-t}{\lambda_{0}^{2}}, \frac{x}{\lambda_{0}}\right) .
$$

Remark 1.2. Case (b) is a direct consequence of the variational characterization of $W$ given by Aubin and Talenti [Aub76], Tal76]. Furthermore, using assumption (1.12), it shows (by continuity of $u$ in $\left.\dot{H}^{1}\right)$ that the assumptions $\int\left|\nabla u\left(t_{0}\right)\right|^{2}<\int|\nabla W|^{2}, \int\left|\nabla u\left(t_{0}\right)\right|^{2}>\int|\nabla W|^{2}$ do not depend on the choice of the initial time $t_{0}$. Of course, this dichotomy does not persist when $E\left(u_{0}\right)>E(W)$.

Remark 1.3. In the supercritical case (IC), our theorem shows that in dimension $N=3$ or $N=4$, an $L^{2}$-solution blows up for negative and positive times. We conjecture that case (C) holds without the assumption " $u_{0} \in L^{2}$ ", i.e. that the only solution with critical energy such that $\int\left|\nabla u_{0}\right|^{2}>\int|\nabla W|^{2}$ and whose interval of definition is not finite is $W^{+}$up to the symmetry of the equation.

Remark 1.4. We expect that the extension of the results of [KM06 to the non-radial case, together with the material in this paper would generalize Theorem 2 to the non-radial case. 
From Bou99a, Bou99b], we know that a solution such that $\|u\|_{S(\mathbb{R})}<\infty$ scatters in $\dot{H}^{1}$ at $\pm \infty$. Cases (a) and (b) of Theorem 2 shows:

Corollary 1.5. Up to the symmetry of the equation, $W$ is the only radial solution such that $E\left(u_{0}\right)=E(W)$ and $\int\left|\nabla u_{0}\right|^{2} \leq \int|\nabla W|^{2}$ which does not scatter in $\dot{H}^{1}$ for neither positive nor negative times.

The behavior exhibited here for $\dot{H}^{1}$-critical NLS is the analogue of the one of the $L^{2}$-critical NLS. For this equation, Merle has shown in Mer93 that a $H^{1}$-solution $u(t)$ at the critical level in $L^{2}$ and such that $x u \in L^{2}$ is either a periodic solution of the form $e^{i \omega t} Q$, an explicit blow-up solution converging to $Q$ after rescaling or a solution scattering at $\pm \infty$.

The outline of the paper is as follows. In Section 2, we use arguments of [KM06] to show the compactness, up to modulation, of a subcritical threshold solution of (1.1) such that $\|u\|_{S(0,+\infty)}=\infty$ (case (a) of Theorem 2). Section 3 is devoted to the proof of the fact that such a solution converges to $W$ as $t \rightarrow+\infty$. In Section \&, we show a similar result for $L^{2}$ super-critical solutions of (1.1) (case (C)). The last ingredient of the proof, which is the object of Section 5, is an analysis of the linearized equation associated to (1.1) near $W$. Both theorems are proven in Section 6 .

\section{COMPACTNESS PROPERTIES FOR NONLINEAR SUBCRITICAL THRESHOLD SOLUTIONS}

In this section we prove a preliminary result related to compactness properties of threshold solutions of (1.1), and which is the starting point of the proofs of Theorems 1 and 2, It is essentially proven in [KM06], Proposition 4.2. We give the proof for the sake of completeness.

If $v$ is a function defined on $\mathbb{R}^{N}$, we will write:

$$
v_{\left[\lambda_{0}\right]}(x)=\frac{1}{\lambda_{0}^{(N-2) / 2}} v\left(\frac{x}{\lambda_{0}}\right), \quad v_{\left[\theta_{0}, \lambda_{0}\right]}=e^{i \theta_{0}} \frac{1}{\lambda_{0}^{(N-2) / 2}} v\left(\frac{x}{\lambda_{0}}\right) .
$$

Proposition 2.1 (Global existence and compactness). Let $u$ be a radial solution of (1.1) and $I=\left(T_{-}, T_{+}\right)$its maximal interval of existence. Assume

$$
E\left(u_{0}\right)=E(W), \quad\left\|u_{0}\right\|_{\dot{H}^{1}}<\|W\|_{\dot{H}^{1}} .
$$

Then

$$
I=\mathbb{R} .
$$

Furthermore, if $\|u\|_{S(0,+\infty)}=\infty$, there exists a map $\lambda$ defined on $[0, \infty)$ such that the set

$$
K_{+}:=\left\{u_{[\lambda(t)]}(t), t \in[0,+\infty)\right\}
$$

is relatively compact in $\dot{H}^{1}$. An analogous assertion holds on $(-\infty, 0]$.

As a corollary we derive the existence of threshold mixed behavior solutions for (1.1) in the subcritical case in $\dot{H}^{1}$-norm.

Corollary 2.2. There exists a solution $w^{-}$of (1.1) defined for $t \in \mathbb{R}$, and such that

$$
\begin{aligned}
& E\left(w^{-}\right)=E(W), \quad\left\|w^{-}(0)\right\|_{\dot{H}^{1}}<\|W\|_{\dot{H}^{1}} \\
& \left\|w^{-}\right\|_{S(0,+\infty)}=\infty, \quad\left\|w^{-}\right\|_{S(-\infty, 0)}<\infty .
\end{aligned}
$$


The crucial point of the proofs of Proposition 2.1 and Corollary 2.2 is a compactness lemma for threshold solutions of (1.1) which is the object of Subsection 2.2. We give a sketch of the proof, which is essentially contained in [KM06], and refer to [KM06] for the details. In Subsections 2.3 and 2.4, we prove respectively Proposition 2.1 and Corollary 2.2, We start with a quick review of the Cauchy Problem for (1.1).

2.1. Preliminaries on the Cauchy Problem. In this subsection we quickly review existence, uniqueness and related results for the Cauchy problem (1.1). See [KM06, Section 2] for the details. In the sequel, $I \ni 0$ is an interval. We first recall the two following relevant function spaces for equation (1.1):

$$
S(I):=L^{\frac{2(N+2)}{N-2}}\left(I \times \mathbb{R}^{N}\right), \quad Z(I):=L^{\frac{2(N+2)}{N-2}}\left(I ; L^{\frac{2 N(N+2)}{N^{2}+4}}\right) .
$$

Note that $Z(I)$ is a Strichartz space for the Schrödinger equation, so that

$$
\left\|\nabla e^{i t \Delta} u_{0}\right\|_{Z(\mathbb{R})} \leq C\left\|u_{0}\right\|_{\dot{H}^{1}}
$$

and that by Sobolev inequality,

$$
\|f\|_{S(I)} \leq C\|\nabla f\|_{Z(I)}
$$

Following [CW90, we say that $u \in C^{0}\left(I, \dot{H}^{1}\left(\mathbb{R}^{N}\right)\right)$ is a solution of (1.1) if for any $J \Subset I$, $u \in S(J),|\nabla u| \in Z(J)$ and

$$
\forall t \in I, \quad u(t)=e^{i t \Delta} u_{0}+i \int_{0}^{t} e^{i(t-s) \Delta}|u(s)|^{p_{c}-1} u(s) d s .
$$

The following holds for such solutions.

\section{Lemma 2.3.}

(a) Uniqueness. Let $u$ and $\tilde{u}$ be two solutions of (1.1) on an interval $I \ni 0$ with the same initial condition $u_{0}$. Then $u=\tilde{u}$.

(b) Existence. For $u_{0} \in \dot{H}^{1}$, there exists an unique solution $u$ of (1.1) defined on a maximal interval of definition $\left(-T_{-}\left(u_{0}\right), T_{+}\left(u_{0}\right)\right)$.

(c) Finite blow-up criterion. Assume that $T_{+}=T_{+}\left(u_{0}\right)<\infty$. Then $\|u\|_{S\left(0, T_{+}\right)}=+\infty$. An analogous result holds for $T_{-}\left(u_{0}\right)$.

(d) Scattering. If $T_{+}\left(u_{0}\right)=\infty$ and $\|u\|_{S([0,+\infty))}<\infty$, there exists $u_{+} \in \dot{H}^{1}$ such that

$$
\lim _{t \rightarrow+\infty}\left\|u(t)-e^{i t \Delta} u_{+}\right\|_{\dot{H}^{1}}=0 .
$$

(e) Continuity. Let $\tilde{u}$ be a solution of (1.1) on $I \ni 0$. Assume that for some constant $A>0$,

$$
\sup _{t \in I}\|\tilde{u}(t)\|_{\dot{H}^{1}}+\|\tilde{u}\|_{S(I)} \leq A .
$$

Then there exist $\varepsilon_{0}=\varepsilon_{0}(A)>0$ and $C_{0}=C_{0}(A)$ such that for any $u_{0} \in \dot{H}^{1}$ with $\left\|\tilde{u}_{0}-u_{0}\right\|_{\dot{H}^{1}}=\varepsilon<\varepsilon_{0}$, the solution $u$ of (1.1) with initial condition $u_{0}$ is defined on $I$ and satisfies $\|u\|_{S(I)} \leq C_{0}$ and $\sup _{t \in I}\|u(t)-\tilde{u}(t)\|_{\dot{H}^{1}} \leq C_{0} \varepsilon$.

(See [CW90], Bou99b], TV05, KM06].) 
Remark 2.4. Precisely, the existence result states that there is an $\varepsilon_{0}>0$ such that if

$$
\left\|e^{i t \Delta} u_{0}\right\|_{S(I)}=\varepsilon<\varepsilon_{0}
$$

then (1.1) has a solution $u$ on $I$ such that $\|u\|_{S(I)} \leq 2 \varepsilon$. In particular, by (2.5) and (2.6), for small initial condition in $\dot{H}^{1}, u$ is globally defined and scatters.

2.2. Compactness or scattering for sequences of threshold $\dot{H}^{1}$-subcritical solutions. The following lemma (closely related to Lemma 4.9 of [KM06]) is a consequence, through the profile decomposition of Keraani Ker01] (which characterizes the defect of compactness of Strichartz estimates for solutions of linear Schrödinger equation), of the scattering of radial subcritical solutions of (1.1) shown in [KM06].

Lemma 2.5. Let $\left(u_{n}^{0}\right)_{n \in \mathbb{N}}$ be a sequence of radial functions in $\dot{H}^{1}$ such that

$$
\forall n, \quad E\left(u_{n}^{0}\right) \leq E(W), \quad\left\|u_{n}^{0}\right\|_{\dot{H}^{1}} \leq\|W\|_{\dot{H}^{1}} .
$$

Let $u_{n}$ be the solution of (1.1) with initial condition $u_{n}^{0}$. Then, up to the extraction of a subsequence of $\left(u_{n}\right)_{n}$, one at least of the following holds:

(a) Compactness. There exists a sequence $\left(\lambda_{n}\right)_{n}$ such that the sequence $\left(\left(u_{n}^{0}\right)_{\left[\lambda_{n}\right]}\right)_{n}$ converges in $\dot{H}^{1}$;

(b) Vanishing for $t \geq 0$. For every $n, u_{n}$ is defined on $[0,+\infty)$ and $\lim _{n \rightarrow+\infty}\left\|u_{n}\right\|_{S(0,+\infty)}=0$;

(c) Vanishing for $t \leq 0$. For every $n, u_{n}$ is defined on $(-\infty, 0]$ and $\lim _{n \rightarrow+\infty}\left\|u_{n}\right\|_{S(-\infty, 0)}=0$;

(d) Uniform scattering. For every $n, u_{n}$ is defined on $\mathbb{R}$. Furthermore, there exists a constant $C$ independent of $n$ such that

$$
\left\|u_{n}\right\|_{S(\mathbb{R})} \leq C .
$$

Sketch of the proof of Lemma 2.5. We will need the following elementary claim (see [KM06, Lemma 3.4]).

Claim 2.6. Let $f \in \dot{H}^{1}$ such that $\|f\|_{\dot{H}^{1}} \leq\|W\|_{\dot{H}^{1}}$. Then

$$
\frac{\|f\|_{\dot{H}^{1}}^{2}}{\|W\|_{\dot{H}^{1}}^{2}} \leq \frac{E(f)}{E(W)} \text {. }
$$

In particular, $E(f)$ is positive.

Remark 2.7. Clearly, $\|u(t)\|_{\dot{H}^{1}}^{2} \geq 2 E(u(t))$, so that Claim 2.6 implies that for solutions of (1.1) satisfying (2.2),

$$
\exists C>0, \forall t, \quad C^{-1}\|u(t)\|_{\dot{H}^{1}}^{2} \leq E(u(t)) \leq C\|u(t)\|_{\dot{H}^{1}}^{2} .
$$

Proof. Let $\Phi(y)=\frac{1}{2} y-\frac{C_{N}^{2^{*}}}{2^{*}} y^{2^{*} / 2}$. Then by Sobolev embedding

$$
\Phi\left(\|f\|_{\dot{H}^{1}}^{2}\right) \leq \frac{1}{2}\|f\|_{\dot{H}^{1}}^{2}-\frac{1}{2^{*}}\|f\|_{L^{2^{*}}}^{2^{*}}=E(f) .
$$

Note that $\Phi$ is concave on $\mathbb{R}_{+}, \Phi(0)=0$ and $\Phi\left(\|W\|_{\dot{H}^{1}}^{2}\right)=E(W)$. Thus

$$
\forall s \in(0,1), \quad \Phi\left(s\|W\|_{\dot{H}^{1}}^{2}\right) \geq s \Phi\left(\|W\|_{\dot{H}^{1}}^{2}\right)=s E(W) .
$$

Taking $s=\frac{\|f\|_{\dot{H}^{1}}^{2}}{\|W\|_{\dot{H}^{1}}^{2}}$ yields the lemma. 
By the lemma of concentration compactness of Keraani (see [Ker01]), there exists a sequence $\left(V_{j}\right)_{j \in \mathbb{N}}$ of solutions of Schrödinger linear equation with initial condition in $\dot{H}^{1}$, and sequences $\left(\lambda_{j n}, t_{j n}\right)_{n \in \mathbb{N}^{*}}, \lambda_{j n}>0, t_{j n} \in \mathbb{R}$, which are pairwise orthogonal in the sense that

$$
j \neq k \Rightarrow \lim _{n \rightarrow+\infty} \frac{\lambda_{k n}}{\lambda_{j n}}+\frac{\lambda_{j n}}{\lambda_{k n}}+\frac{\left|t_{j n}-t_{k n}\right|}{\lambda_{j n}^{2}}=+\infty
$$

such that for all $J$

$$
\begin{gathered}
u_{n}^{0}=\sum_{j=1}^{J} V_{j}\left(s_{j n}\right)_{\left[\lambda_{j n}\right]}+w_{n}^{J}, \text { with } s_{j n}=\frac{-t_{j n}}{\lambda_{j n}^{2}}, \\
\lim _{J \rightarrow+\infty} \limsup _{n \rightarrow+\infty}\left\|e^{i t \Delta} w_{n}^{J}\right\|_{S(\mathbb{R})}=0, \\
\left\|u_{n}^{0}\right\|_{\dot{H}^{1}}^{2}=\sum_{j=1}^{J}\left\|V_{j}\right\|_{\dot{H}^{1}}^{2}+\left\|w_{n}^{J}\right\|_{\dot{H}^{1}}^{2}+o(1) \text { as } n \rightarrow+\infty, \\
E\left(u_{n}^{0}\right)=\sum_{j=1}^{J} E\left(V_{j}\left(s_{j n}\right)\right)+E\left(w_{n}^{J}\right)+o(1) \text { as } n \rightarrow+\infty .
\end{gathered}
$$

If all the $V_{j}$ 's are identically 0 , then by (2.10), $\left\|e^{i t \Delta} u_{n}^{0}\right\|_{S(\mathbb{R})}$ tends to 0 as $n$ tends to infinity, and the sequence $\left(u_{n}\right)_{n}$ satisfies simultaneously (b), (ㄷ) and (d). Thus we may assume without loss of generality that $V_{1} \neq 0$. Furthermore, by assumption (2.8) and by (2.11), $\left\|V_{j}\right\|_{\dot{H}^{1}} \leq\|W\|_{\dot{H}^{1}}$ and for large $n,\left\|w_{n}^{J}\right\|_{\dot{H}^{1}}<\|W\|_{\dot{H}^{1}}$, which implies by Claim 2.6 that the energies $E\left(V_{j}\left(s_{j n}\right)\right)$ and $E\left(w_{n}^{J}\right)$ are nonnegative, and thus that $E\left(V_{j}\left(s_{j n}\right)\right) \leq E(W)$. Extracting once again a subsequence if necessary, we distinguish two cases.

First case:

$$
\lim _{n \rightarrow+\infty} E\left(V_{1}\left(s_{1 n}\right)\right)=E(W) .
$$

By assumption (2.8) and by (2.12) (all the energies being nonnegative for large $n), E\left(V_{j}\left(s_{j n}\right)\right.$ ) $(j \geq 2)$, and $E\left(w_{n}^{J}\right)$ tend to 0 as $n$ tends to infinity. Thus by Claim [2.6, for $j \geq 2, V_{j}=0$, and $w_{n}^{J}=w_{n}^{1}$ tends to 0 in $\dot{H}^{1}$. As a consequence

$$
u_{n}^{0}=V_{1}\left(s_{1 n}\right)_{\left[\lambda_{1 n}\right]}+o(1) \text { in } \dot{H}^{1}, n \rightarrow+\infty .
$$

Up to the extraction of a subsequence, $s_{1 n}$ converges to some $s \in[-\infty,+\infty]$. If $s \in \mathbb{R}$, It is easy to see that we are in case (a) (compactness up to modulation) of Lemma 2.5. If $s=+\infty$, then $\lim _{n \rightarrow+\infty}\left\|e^{i t \Delta} u_{n}^{0}\right\|_{S(0,+\infty)}=0$, so that by existence theory for (1.1) (see Remark 2.4) case (b) holds. Similarly, if $s=-\infty$ case (ㄷ) holds.

Second case:

$$
\exists \varepsilon_{1}, 0<\varepsilon_{1}<E(W) \text { and } \forall n, E\left(V_{1}\left(s_{1 n}\right)\right) \leq E(W)-\varepsilon_{1} .
$$

Here we are exactly in the situation of the first case of [KM06, Lemma 4.9]. We refer to the proof of this lemma for the details. Recall that for large $n$, all the energies are nonnegative in (2.12). Thus, in view of (2.12) (and of Claim 2.6 for the second inequality)

$$
\limsup _{n \rightarrow+\infty} E\left(V_{j}\left(s_{j n}\right)\right) \leq \varepsilon_{1}<E(W), \quad\left\|V_{j}(0)\right\|_{\dot{H}^{1}}<\|W\|_{\dot{H}^{1}} .
$$


Furthermore by assumption (2.8) and by (2.11)

$$
\sum_{j \geq 1}\left\|V_{j}\left(s_{j n}\right)\right\|_{\dot{H}^{1}}^{2} \leq\|W\|_{\dot{H}^{1}}^{2} .
$$

Thus, according to the results of [KM06] and the Cauchy problem theory for (1.1), $u_{n}^{0}$ is, up to the small term $w_{n}^{J}$, a sum (2.9) of terms $U_{j n}^{0}=V_{j}\left(s_{j n}\right)_{\left[\lambda_{j n}\right]}$ that are all initial conditions of a solution $U_{j n}$ of (1.1) satisfying an uniform bound $\left\|U_{j n}\right\|_{S(\mathbb{R})} \leq c_{j}$ (with $\sum c_{j}^{2}$ finite by (2.15) $)$. Using the pairwise orthogonality of the sequences $\left(\lambda_{j n}, t_{j n}\right)_{n \in \mathbb{N}^{*}}$, together with a longtime perturbation result for (1.1), it is possible to show that for some constant $C$ independant of $n$,

$$
\left\|u_{n}\right\|_{S(\mathbb{R})} \leq C,
$$

that is that case (d) of the Lemma holds. Up to the technical proof of this fact, which we omit, the proof of Lemma 2.5 is complete.

2.3. Compactness up to modulation and global existence of threshold solutions. We now prove Proposition 2.1.

Step 1: compactness. We start by showing the compactness up to modulation of the threshold solution $u$. In Step 2 we will show that $u$ is defined on $\mathbb{R}$.

Lemma 2.8. Let $u$ be a solution of (1.1) of maximal interval of definition $\left[0, T_{+}\right)$such that $E\left(u_{0}\right)=E(W),\left\|u_{0}\right\|_{\dot{H}^{1}}<\|W\|_{\dot{H}^{1}}$ and

$$
\|u\|_{S\left(0, T_{+}\right)}=+\infty \text {. }
$$

Then there exists a function $\lambda$ on $\left[0, T_{+}\right)$such that the set

$$
K_{+}:=\left\{u_{[\lambda(t)]}(t), t \in\left[0, T_{+}\right)\right\}
$$

is relatively compact in $\dot{H}^{1}$.

Proof. The proof is similar to the one in [KM06. The main point of the proof is to show that for every sequence $\left(t_{n}\right)_{n}, t_{n} \in\left[0, T_{+}\right)$, there exists, up to the extraction of a subsequence, a sequence $\left(\lambda_{n}\right)_{n}$ such that $\left(u_{\left[\lambda_{n}\right]}\left(t_{n}\right)\right)$ converges in $\dot{H}^{1}$. By continuity of $u$, we just have to consider the case $\lim _{n} t_{n}=T_{+}$.

Let us use Lemma 2.5 for the sequence $u_{n}^{0}=u\left(t_{n}\right)$. We must show that we are in case (a). Clearly, cases (b) (vanishing for $t \geq 0$ ) and (d) (uniform scattering) are excluded by the assumption that $\|u\|_{S\left(0, T_{+}\right)}$is infinite. Furthermore, $\|u\|_{S\left(0, t_{n}\right)}=\left\|u_{n}\right\|_{S\left(-t_{n}, 0\right)}$ (where $u_{n}$ is the solution of (1.1) with initial condition $u_{n}^{0}$ ) so that case (ㄷ) would imply that $\|u\|_{S\left(0, t_{n}\right)}$ tends to 0 , i.e that $u$ is identically 0 which contradicts our assumptions. Thus case (国) holds: there exists, up to the extraction of a subsequence, a sequence $\left(\lambda_{n}\right)_{n}$ such that $\left(u_{\left[\lambda_{n}\right]}\left(t_{n}\right)\right)_{n}$ converges.

The existence of $\lambda(t)$ such that the set $K_{+}$defined by (2.16) is relatively compact is now classical. Indeed

$$
\forall t \in\left[0, T_{+}\right), \quad 2 E(W)=2 E(u(t)) \leq\|u(t)\|_{\dot{H}^{1}}^{2} \leq\|W\|_{\dot{H}^{1}}^{2} .
$$

Fixing $t \in\left[0, T_{+}\right)$, define

$$
\lambda(t):=\sup \left\{\lambda>0, \text { s.t. } \int_{|x| \leq 1 / \lambda}|\nabla u|^{2}(t, x) d x=E(W)\right\} .
$$


By (2.17), $0<\lambda(t)<\infty$. Let $\left(t_{n}\right)_{n}$ be a sequence in $\left[0, T_{+}\right)$. As proven before, up to the extraction of a subsequence, there exists a sequence $\left(\lambda_{n}\right)_{n}$ such that $\left(u_{\left[\lambda_{n}\right]}\left(t_{n}\right)\right)_{n}$ converges in $\dot{H}^{1}$ to a function $v_{0}$ of $\dot{H}^{1}$. One may check directly, using (2.17), that for a constant $C>0$,

$$
C^{-1} \lambda\left(t_{n}\right) \leq \lambda_{n} \leq C \lambda\left(t_{n}\right),
$$

which shows (extracting again subsequences if necessary) the convergence of $\left(u_{\left[\lambda\left(t_{n}\right)\right]}\left(t_{n}\right)\right)_{n}$ in $\dot{H}^{1}$. The compactness of $\bar{K}_{+}$is proven, which concludes the proof of Lemma 2.8 .

Step 2: global existence. To complete the proof of Proposition 2.1, it remains to show that the maximal time of existence $T_{+}=T_{+}\left(u_{0}\right)$ is infinite. Here we use an argument in [KM06]. Assume

$$
T_{+}<\infty
$$

and consider a sequence $t_{n}$ that converges to $T_{+}$. By the finite blow-up criterion of Lemma 2.3, $\|u\|_{S\left(0, T_{+}\right)}=+\infty$. By Lemma 2.8, there exists $\lambda(t)$ such that the set $K_{+}$defined by (2.16) is relatively compact in $\dot{H}^{1}$.

If there exists a sequence $\left(t_{n}\right)_{n}$ converging to $T_{+}$such that $\lambda\left(t_{n}\right)$ has a finite limit $\lambda_{0} \geq 0$, then it is easy to show, using the compactness of $\left(u_{\left[\lambda\left(t_{n}\right)\right]}\left(t_{n}\right)\right)_{n}$ and the scaling invariance of (1.1) that $u$ is defined in a neighborhood of $T_{+}$, which contradicts the fact that $T_{+}$is the maximal positive time of definition of $u$. Thus we may assume

$$
\lim _{t \rightarrow T_{+}} \lambda(t)=+\infty
$$

Consider a positive radial function $\psi$ on $\mathbb{R}^{N}$, such that $\psi=1$ if $|x| \leq 1$ and $\psi=0$ if $|x| \geq 2$. Define, for $R>0$ and $t \in\left[0, T_{+}\right)$,

$$
F_{R}(t):=\int_{\mathbb{R}^{N}}|u(t, x)|^{2} \psi\left(\frac{x}{R}\right) d x .
$$

By (2.20), the relative compactness of $K_{+}$in $\dot{H}^{1}$ and Sobolev inequality, for all $r_{0}>0$, $\int_{|x| \geq r_{0}}|u(t, x)|^{2^{*}} d x$ tends to 0 as $t$ tends to $T_{+}$. Thus, by Hölder and Hardy inequalities

$$
\lim _{t \rightarrow T_{+}} F_{R}(t)=0 \text {. }
$$

Using equation (1.1), $F_{R}^{\prime}(t)=\frac{2}{R} \operatorname{Im} \int u(x) \nabla \bar{u}(x)(\nabla \psi)\left(\frac{x}{R}\right) d x$, which shows (using Cauchy-Schwarz and Hardy inequalities) that $\left|F_{R}^{\prime}(t)\right| \leq C\|u(t)\|_{\dot{H}^{1}}^{2} \leq C_{0}$, where $C_{0}$ is a constant which is independent of $R$. Fixing $t \in\left[0, T_{+}\right)$, we see that

$$
\forall T \in\left[0, T_{+}\right), \quad\left|F_{R}(t)-F_{R}(T)\right| \leq C_{0}|t-T| .
$$

Thus, letting $T$ tends to $T_{+}$, and using (2.21), $\left|F_{R}(t)\right| \leq C_{0}\left|t-T_{+}\right|$. Letting $R$ tends to infinity, one gets that $u(t)$ is in $L^{2}\left(\mathbb{R}^{N}\right)$ and satisfies

$$
\int_{\mathbb{R}^{N}}|u(t, x)|^{2} d x \leq C\left|t-T_{+}\right| .
$$

By conservation of the $L^{2}$ norm, we get that $u_{0}=0$ which contradicts the fact that $E\left(u_{0}\right)=$ $E(W)$. This completes the proof that $T_{+}=+\infty$. By a similar argument, $T_{-}=-\infty$. The proof of Proposition 2.1 is complete. 
2.4. Existence of mixed behavior solutions. We now prove Corollary 2.2 ,

Let $v_{n}^{0}=\left(1-\frac{1}{n}\right) W$ and $v_{n}$ the solution of (1.1) with initial condition $v_{n}^{0}$. One may check that $\left\|v_{n}^{0}\right\|_{\dot{H}^{1}}<\|W\|_{\dot{H}^{1}}$ and $E\left(v_{n}^{0}\right)<E(W)$. By the results in [KM06]

$$
T_{+}\left(v_{n}^{0}\right)=T_{-}\left(v_{n}^{0}\right)=\infty, \quad\left\|v_{n}\right\|_{S(\mathbb{R})}<\infty .
$$

Since $\|W\|_{S(\mathbb{R})}=\infty,\left\|v_{n}\right\|_{S(\mathbb{R})}$ tends to $+\infty$ by Lemma 2.3 (国). Chose $t_{n}$ such that the solution $u_{n}(\cdot)=v_{n}\left(\cdot+t_{n}\right)$ of (1.1) satisfies

$$
\left\|u_{n}\right\|_{S(-\infty, 0)}=1
$$

Therefore

$$
\left\|u_{n}\right\|_{S(0,+\infty)} \underset{n \rightarrow+\infty}{\longrightarrow}+\infty \text {. }
$$

Let us use Lemma 2.5, By (2.23) and (2.24), cases (마), (드) and (d) are excluded. Extracting a subsequence from $\left(u_{n}\right)_{n}$, there exists a sequence $\left(\lambda_{n}\right)_{n}$ such that $\left(u_{n}\right)_{\left[\lambda_{n}\right]}$ converges in $\dot{H}^{1}$. Rescaling each $u_{n}$ if necessary (which preserves properties (2.23) and (2.24)), we may assume that the sequence $\left(u_{n}^{0}\right)_{n}$ converges in $\dot{H}^{1}$ to some $w_{0}^{-}$. Let $w^{-}$be the solution of (1.1) such that $w^{-}(0)=w_{0}^{-}$. Clearly

$$
E\left(w_{0}^{-}\right)=E(W), \quad\left\|w_{0}^{-}\right\|_{\dot{H}^{1}} \leq\|W\|_{\dot{H}^{1}} .
$$

Thus by Proposition 2.1, $w^{-}$is defined on $\mathbb{R}$.

Fix a large integer $n$. By (2.23) and Lemma 2.3 (国) with $I=(-\infty, 0], \tilde{u}=u_{n}$, and $u_{0}=w_{0}^{-}$, we get

$$
\left\|w^{-}\right\|_{S(-\infty, 0)}<\infty
$$

Assume that $\left\|w^{-}\right\|_{S(0,+\infty)}$ is finite. Using again Lemma 2.3 (ㅌ) with $I=[0,+\infty), \tilde{u}=w^{-}$and $u_{0}=u_{n}(0)$, we would get that $\left\|u_{n}\right\|_{S(0,+\infty)}$ is bounded independently of $n$, contradicting (2.24). Thus

$$
\left\|w^{-}\right\|_{S(0,+\infty)}=\infty
$$

The proof is complete.

Remark 2.9. The above proof gives a general proof of existence of a mixed-behavior solution at the threshold. In Section [6, we will give another proof by a fixed point argument, which also works in the supercritical case $\left\|u_{0}\right\|_{\dot{H}^{1}}>\|W\|_{\dot{H}^{1}}$.

\section{Convergence to $W$ in the subcritical case}

In this section, we consider a threshold subcritical radial solution $u$ of (1.1), satisfying

$$
\begin{gathered}
E\left(u_{0}\right)=E(W), \quad\left\|u_{0}\right\|_{\dot{H}^{1}}^{2}<\|W\|_{\dot{H}^{1}}^{2} \\
\|u\|_{S(0,+\infty)}=+\infty .
\end{gathered}
$$

We will show:

Proposition 3.1. Let $u$ be a radial solution of (1.1) satisfying (3.1) and (3.2). Then there exist $\theta_{0} \in \mathbb{R}, \mu_{0}>0$ and $c, C>0$ such that

$$
\forall t \geq 0, \quad\left\|u(t)-W_{\left[\theta_{0}, \mu_{0}\right]}\right\|_{\dot{H}^{1}} \leq C e^{-c t} .
$$

(See (2.1) for the definition of $\left.W_{\left[\theta_{0}, \mu_{0}\right]}\right)$. As a corollary of the preceding proposition and a step of its proof we get the following result, which completes the proof of the third assertion in (1.10) of Theorem 2. 
Corollary 3.2. There is no solution $u$ of (1.1) satisfying (3.1) and

$$
\|u\|_{S(-\infty, 0)}=\|u\|_{S(0,+\infty)}=+\infty .
$$

Let

$$
\mathrm{d}(f):=\left|\|f\|_{\dot{H}^{1}}^{2}-\|W\|_{\dot{H}^{1}}^{2}\right| .
$$

The key to proving Proposition 3.1 is to show

$$
\lim _{t \rightarrow+\infty} \mathrm{d}(u(t))=0 .
$$

Our starting point of the proof of (3.5) is to prove the existence of a sequence $t_{n}$ going to infinity such that $\mathrm{d}\left(u\left(t_{n}\right)\right)$ goes to 0 (Subsection 3.1). After giving, in Subsection 3.2, some useful results on the modulation of threshold solutions with respect to the manifold $\left\{W_{[\theta, \mu]}, \mu>0, \theta \in \mathbb{R}\right\}$, we will show the full convergence (3.5) and finish the proof of Proposition 3.1 and Corollary [3.2.

\subsection{Convergence to $W$ for a sequence.}

Lemma 3.3. Let $u$ be a radial solution of (1.1) satisfying (3.1), (3.2), and thus defined on $\mathbb{R}$ by Proposition [2.1. Then

$$
\lim _{t \rightarrow+\infty} \frac{1}{T} \int_{0}^{T} \mathrm{~d}(u(t)) d t=0 .
$$

Corollary 3.4. Under the assumptions of Lemma 3.3, there exists a sequence $t_{n} \rightarrow+\infty$ such that $\mathrm{d}\left(u\left(t_{n}\right)\right)$ tends to 0 .

Proof of Lemma 3.3. Let $u$ be a solution of (1.1) satisfying (3.1) and (3.2). According to Proposition [2.1, there exists a function $\lambda(t)$ such that $K_{+}:=\left\{u_{[\lambda(t)]}(t), t \geq 0\right\}$ is relatively compact in $\dot{H}^{1}$.

The proof take three steps.

Step 1: virial argument. Let $\varphi$ be a radial, smooth, cut-off function on $\mathbb{R}^{N}$ such that

$$
\varphi(x)=|x|^{2}, 0 \leq|x| \leq 1, \quad \varphi(x)=0,|x| \geq 2 .
$$

Let $R>0$ and $\varphi_{R}(x)=R^{2} \varphi\left(\frac{x}{R}\right)$, so that $\varphi_{R}(x)=|x|^{2}$ for $|x| \leq R$. Consider the quantity (which is the time-derivative of the localized variance)

$$
G_{R}(t):=2 \operatorname{Im} \int \bar{u}(t) \nabla u(t) \cdot \nabla \varphi_{R}, \quad t \in \mathbb{R} .
$$

Let us show

$$
\begin{gathered}
\exists C_{*}>0, \forall t \in \mathbb{R}, \quad\left|G_{R}(t)\right| \leq C_{*} R^{2} \\
\forall \varepsilon>0, \exists \rho_{\varepsilon}>0, \forall R>0, \forall t \geq 0, \quad R \lambda(t) \geq \rho_{\varepsilon} \Longrightarrow G_{R}^{\prime}(t) \geq \frac{16}{N-2} \mathrm{~d}(u(t))-\varepsilon .
\end{gathered}
$$

Using that $|x| \leq 2 R$ on the support of $\varphi_{R}$ and that $\left|\nabla \varphi_{R}\right| \leq C R$, we get

$$
\forall t \in \mathbb{R}, \quad\left|G_{R}(t)\right| \leq C R^{2} \int_{\mathbb{R}^{N}} \frac{1}{|x|}|u(t)||\nabla u(t)| \leq C R^{2}\left(\int_{\mathbb{R}^{N}}|\nabla u(t)|^{2}\right)^{1 / 2}\left(\int_{\mathbb{R}^{N}} \frac{1}{|x|^{2}}|u(t)|^{2}\right)^{1 / 2},
$$


which yields (3.7), as a consequence of Hardy's inequality and $\|u(t)\|_{\dot{H}^{1}} \leq\|W\|_{\dot{H}^{1}}$. To show (3.8), we will use the compactness of $\bar{K}_{+}$. By direct computation

$$
G_{R}^{\prime}(t)=\frac{16}{N-2} \mathrm{~d}(u(t))+A_{R}(u(t))
$$

where

$$
\begin{aligned}
A_{R}(u):=\int_{|x| \geq R}\left|\partial_{r} u\right|^{2} & \left(4 \frac{d^{2} \varphi_{R}}{d r^{2}}-8\right) r^{N-1} d r \\
& +\int_{|x| \geq R}|u|^{2^{*}}\left(-\frac{4}{N} \Delta \varphi_{R}+8\right) r^{N-1} d r-\int|u|^{2}\left(\Delta^{2} \varphi_{R}\right) r^{N-1} d r .
\end{aligned}
$$

Indeed, an explicit calculation yields, together with equation (1.1)

$$
\begin{aligned}
G_{R}^{\prime}(t) & =4 \int\left|\frac{\partial u}{\partial r}\right|^{2} \frac{d^{2} \varphi_{R}}{d r^{2}} r^{N-1} d r-\frac{4}{N} \int|u|^{2^{*}}\left(\Delta \varphi_{R}\right) r^{N-1} d r-\int|u|^{2}\left(\Delta^{2} \varphi_{R}\right) r^{N-1} d r \\
& =8\left(\int_{\mathbb{R}^{N}}|\nabla u(t)|^{2}-\int_{\mathbb{R}^{N}}|u(t)|^{2 *}\right)+A_{R}(u(t)),
\end{aligned}
$$

and as a consequence of assumption (3.1), $\int|\nabla u(t)|^{2}-\int|u(t)|^{2 *}=\frac{2}{N-2} \mathrm{~d}(u(t))$ which yields (3.9). According to (3.9), we must show

$$
\forall \varepsilon>0, \exists \rho_{\varepsilon}>0, \forall R>0, \forall t \geq 0, \quad R \lambda(t) \geq \rho_{\varepsilon} \Longrightarrow\left|A_{R}(u(t))\right|<\varepsilon .
$$

We have $\left|\partial_{r}^{2} \varphi_{R}\right|+\left|\Delta \varphi_{R}\right| \leq C$ and $\left|\Delta^{2} \varphi_{R}\right| \leq C / R^{2}$. Thus by (3.10),

$$
\begin{aligned}
& \left|A_{R}(u(t))\right| \leq \int_{|x| \geq R}|\nabla u(t, x)|^{2}+|u(x)|^{2^{*}}+\frac{1}{|x|^{2}}|u(t, x)|^{2} d x \\
& \left|A_{R}(u(t))\right| \leq \int_{|y| \geq R \lambda(t)}\left|\nabla u_{[\lambda(t)]}(t, y)\right|^{2}+\left|u_{[\lambda(t)]}(y)\right|^{2^{*}}+\frac{1}{|y|^{2}}\left|u_{[\lambda(t)]}(t, y)\right|^{2} d x,
\end{aligned}
$$

The set $\bar{K}_{+}$being compact in $\dot{H}^{1}$, we get (3.11) in view of Hardy and Sobolev inequalities. The proof of (3.8) is complete.

Step 2: a bound from below for $\lambda(t)$. The next step is to show

$$
\lim _{t \rightarrow+\infty} \sqrt{t} \lambda(t)=+\infty \text {. }
$$

We argue by contradiction. If (3.13) does not hold, there exists $t_{n} \rightarrow+\infty$ such that

$$
\lim _{n \rightarrow+\infty} \sqrt{t_{n}} \lambda\left(t_{n}\right)=\tau_{0}<\infty .
$$

Consider the sequence $\left(v_{n}\right)_{n}$ of solutions of (1.1) defined by

$$
v_{n}(\tau, y)=\frac{1}{\lambda\left(t_{n}\right)^{\frac{N-2}{2}}} u\left(t_{n}+\frac{\tau}{\lambda^{2}\left(t_{n}\right)}, \frac{y}{\lambda\left(t_{n}\right)}\right) .
$$

By the compactness of $\bar{K}_{+}$, one may assume that $\left(v_{n}(0)\right)_{n}$ converges in $\dot{H}^{1}$ to some function $v_{0}$. Let $v(\tau)$ be the solution of (1.1) with initial condition $v_{0}$ at time $\tau=0$. Clearly $E\left(v_{0}\right)=E(W)$ and $\left\|v_{0}\right\|_{\dot{H}^{1}} \leq\|W\|_{\dot{H}^{1}}$. From Proposition 2.1, $v$ is defined on $\mathbb{R}$. Furthermore, by (3.14) and the uniform continuity of the flow of equation (1.1) (Lemma 2.3 (国) )

$$
\lim _{n \rightarrow+\infty} v_{n}\left(-\lambda^{2}\left(t_{n}\right) t_{n}\right)=v\left(-\tau_{0}\right) \text {, in } \dot{H}^{1} \text {. }
$$


By (3.15),

$$
v_{n}\left(-\lambda^{2}\left(t_{n}\right) t_{n}, y\right)=\frac{1}{\lambda^{(N-2) / 2}\left(t_{n}\right)} u_{0}\left(\frac{y}{\lambda\left(t_{n}\right)}\right) .
$$

Assumption (3.14) implies that $\lambda\left(t_{n}\right)$ tends to 0 , which shows that $v_{n}\left(-\lambda^{2}\left(t_{n}\right) t_{n}\right) \rightarrow 0$ weakly in $\dot{H}^{1}$, contradicting (3.16) unless $v\left(-\tau_{0}\right)=0$. This is excluded by the fact that $E\left(v\left(-\tau_{0}\right)\right)=$ $E(W)$, which concludes the proof of (3.13).

Step 3: conclusion of the proof. Fix $\varepsilon>0$. We will use the estimates (3.7) and (3.8) of Step 1 with an appropriate choice of $R$. Consider the positive number $\rho_{\varepsilon}$ given by (3.8). Take $\varepsilon_{0}$ and $M_{0}$ such that

$$
2 C_{*} \varepsilon_{0}^{2}=\varepsilon, \quad M_{0} \varepsilon_{0}=\rho_{\varepsilon},
$$

where $C_{*}$ is the constant of inequality (3.7). By Step 2 there exists $t_{0}$ such that

$$
\forall t \geq t_{0}, \quad \lambda(t) \geq \frac{M_{0}}{\sqrt{t}} .
$$

Consider, for $T \geq t_{0}$

$$
R:=\varepsilon_{0} \sqrt{T} .
$$

If $t \in\left[t_{0}, T\right]$, then the definitions of $R, M_{0}$ and $t_{0}$ imply $R \lambda(t) \geq \varepsilon_{0} \sqrt{T} \frac{M_{0}}{\sqrt{t}} \geq \rho_{\varepsilon}$. Integrating (3.8) between $t_{0}$ and $T$ and using estimate (3.7) on $G_{R}$, we get, by the choice of $\varepsilon_{0}$ and $R$

$$
\frac{16}{N-2} \int_{t_{0}}^{T}|\mathrm{~d}(u(t))| d t \leq 2 C_{*} R^{2}+\varepsilon\left(T-t_{0}\right) \leq 2 C_{*} R^{2}+\varepsilon T \leq \frac{\varepsilon}{\varepsilon_{0}^{2}} \varepsilon_{0}^{2} T+\varepsilon T \leq 2 \varepsilon T .
$$

Letting $T$ tends to $+\infty$,

$$
\limsup _{T \rightarrow+\infty} \frac{1}{T} \int_{0}^{T}|\mathrm{~d}(u(t))| d t \leq \frac{N-2}{8} \varepsilon,
$$

which concludes the proof of Lemma 3.3 .

3.2. Modulation of threshold solutions. Let $f$ be in $\dot{H}^{1}$ such that $E(f)=E(W)$. The variational characterization of $W$ Aub76, Tal76, Lio85, shows

$$
\inf _{\substack{\theta \in \mathbb{R} \\ \mu>0}}\left\|f_{[\theta, \mu]}-W\right\|_{\dot{H}^{1}} \leq \varepsilon(\mathrm{d}(f)), \quad \lim _{\delta \rightarrow 0^{+}} \varepsilon(\delta)=0,
$$

where $\mathrm{d}(f)$ is defined in (3.4). We introduce here a choice of the modulation parameters $\theta$ and $\mu$ for which the quantity $\mathrm{d}(f)$ controls linearly $\left\|f_{[\theta, \mu]}-W\right\|_{\dot{H}^{1}}$ and other relevant parameters of the problem. This choice is made through two orthogonality conditions given by the two groups of transformations $f \mapsto e^{i \theta} f, \theta \in \mathbb{R}$ and $f \mapsto f_{[\mu]}, \mu>0$. This decomposition is then applied to solutions of (1.1). Let us start with a few notations.

Since $W$ is a critical point of $E$, we have the following development of the energy near $W$ :

$$
E(W+g)=E(W)+Q(g)+O\left(\|g\|_{\dot{H}^{1}}^{3}\right), \quad g \in \dot{H}^{1},
$$

where $Q$ is the quadratic form on $\dot{H}^{1}$ defined by

$$
Q(g):=\frac{1}{2} \int|\nabla g|^{2}-\frac{1}{2} \int W^{p_{c}-1}\left(p_{c}(\operatorname{Re} g)^{2}+(\operatorname{Im} g)^{2}\right) .
$$


Let us specify an important coercivity property of $Q$. Consider the three orthogonal directions $W, i W$ and $W_{1}:=\frac{N-2}{2} W+x \cdot \nabla W$ in the real Hilbert space $\dot{H}^{1}=\dot{H}^{1}\left(\mathbb{R}^{N}, \mathbb{C}\right)$. Note that

$$
i W=\frac{d}{d \theta}\left(e^{i \theta} W\right)_{\lceil\theta=0}, \quad W_{1}=-\frac{d}{d \lambda}\left(W_{[\lambda]}\right)_{\lceil\lambda=1} .
$$

Let $H:=\operatorname{span}\left\{W, i W, W_{1}\right\}$ and $H^{\perp}$ its orthogonal subspace in $\dot{H}^{1}$ for the usual scalar product. Then

$$
Q(W)=-\frac{2}{(N-2) C_{N}^{N}}, \quad Q_{\left\lceil\operatorname{span}\left\{i W, W_{1}\right\}\right.}=0,
$$

where $C_{N}$ is the best Sobolev constant in dimension $N$. The first assertion follows from direct computation and the fact that $\|W\|_{L^{2^{*}}}^{2^{*}}=\|W\|_{\dot{H}^{1}}^{2}=\frac{1}{C_{N}^{N}}$. The second assertion is an immediate consequence of (3.17), (3.18), and the invariance of $E$ by the transformations $f \mapsto f_{[\theta, \lambda]}$. The quadratic form $Q$ is nonpositive on $H$. By the following claim, $Q$ is positive definite on $H^{\perp}$.

Claim 3.5. There is a constant $\tilde{c}>0$ such that for all radial function $\tilde{f}$ in $H^{\perp}$

$$
Q(\tilde{f}) \geq \tilde{c}\|\tilde{f}\|_{\dot{H}^{1}}^{2} .
$$

Proof. Let $\tilde{f}_{1}:=\operatorname{Re} \tilde{f}, \tilde{f}_{2}:=\operatorname{Im} \tilde{f}$. We have

$$
Q(\tilde{f})=\frac{1}{2} \int_{\mathbb{R}^{N}}\left|\nabla \tilde{f}_{1}\right|^{2}-\frac{p_{c}}{2} \int_{\mathbb{R}^{N}} W^{p_{c}-1}\left|\tilde{f}_{1}\right|^{2}+\frac{1}{2} \int_{\mathbb{R}^{N}}\left|\nabla \tilde{f}_{2}\right|^{2}-\frac{1}{2} \int_{\mathbb{R}^{N}} W^{p_{c}-1}\left|\tilde{f}_{2}\right|^{2} .
$$

The inequality

$$
\exists c_{1}>0, \forall \tilde{f}_{1} \in\left\{W, W_{1}\right\}^{\perp}, \quad \frac{1}{2} \int_{\mathbb{R}^{N}}\left|\nabla \tilde{f}_{1}\right|^{2}-\frac{p_{c}}{2} \int_{\mathbb{R}^{N}} W^{p_{c}-1}\left|\tilde{f}_{1}\right|^{2} \geq c_{1} \int_{\mathbb{R}^{N}}\left|\nabla \tilde{f}_{1}\right|^{2}
$$

is known. We refer to Rey90, Appendix D] for a proof in a slightly different context, but which readily extends to our case. It remains to show

$$
\exists c_{2}>0, \forall \tilde{f}_{2} \in \dot{H}^{1}, \quad \tilde{f}_{2} \perp W \Longrightarrow \frac{1}{2} \int_{\mathbb{R}^{N}}\left|\nabla \tilde{f}_{2}\right|^{2}-\frac{1}{2} \int_{\mathbb{R}^{N}} W^{p_{c}-1}\left|\tilde{f}_{2}\right|^{2} \geq c_{2} \int_{\mathbb{R}^{N}}\left|\nabla \tilde{f}_{2}\right|^{2} .
$$

Indeed by Hölder and Sobolev inequality we have, for any real-valued $v \in \dot{H}^{1}$

$$
\begin{aligned}
\int_{\mathbb{R}^{N}}|\nabla v|^{2}-\int_{\mathbb{R}^{N}} W^{p_{c}-1} v^{2} & \geq \int_{\mathbb{R}^{N}}|\nabla v|^{2}-\left(\int_{\mathbb{R}^{N}} W^{p_{c}+1}\right)^{\frac{p_{c}-1}{p_{c}+1}}\left(\int v^{p_{c}+1}\right)^{\frac{2}{p+1}} \\
& \geq\left\{1-\left(\frac{1}{C_{N}^{N}}\right)^{\frac{p_{c}-1}{p_{c}+1}} C_{N}^{2}\right\} \int_{\mathbb{R}^{N}}|\nabla v|^{2} \geq 0
\end{aligned}
$$

with equality if and only if $v \in \operatorname{span}(W)$. This shows that $\int_{\mathbb{R}^{N}}\left|\nabla \tilde{f}_{2}\right|^{2}-\int_{\mathbb{R}^{N}} W^{p_{c}-1}\left|\tilde{f}_{2}\right|^{2}>0$ for $\tilde{f}_{2} \neq 0, \tilde{f}_{2} \perp W$. Noting that the quadratic form $\int_{\mathbb{R}^{N}}|\nabla \cdot|^{2}-\int_{\mathbb{R}^{N}} W^{p_{c}-1}|\cdot|^{2}$ is a compact perturbation of $\int_{\mathbb{R}^{N}}|\nabla \cdot|^{2}$, it is easy to derive (3.20), using a straightforward compactness argument that we omit here.

The following lemma, proven in Appendix 17.1, is a consequence of the Implicit Function Theorem. 
Lemma 3.6. There exists $\delta_{0}>0$ such that for all $f$ in $\dot{H}^{1}$ with $E(f)=E(W), \mathrm{d}(f)<\delta_{0}$, there exists a couple $(\theta, \mu)$ in $\mathbb{R} \times(0,+\infty)$ with

$$
f_{[\theta, \mu]} \perp i W, \quad f_{[\theta, \mu]} \perp W_{1} .
$$

The parameters $\theta$ and $\mu$ are unique in $\mathbb{R} / 2 \pi \mathbb{Z} \times \mathbb{R}$, and the mapping $f \mapsto(\theta, \mu)$ is $C^{1}$.

Let $u$ be a solution of (1.1) on an interval $I$ such that $E\left(u_{0}\right)=E(W)$, and, on $I, \mathrm{~d}(u(t))<\delta_{0}$. According to Lemma 3.6, there exist real parameters $\theta(t), \mu(t)>0$ such that

$$
\begin{aligned}
& u_{[\theta(t), \mu(t)]}(t)=(1+\alpha(t)) W+\tilde{u}(t), \\
& \text { where } 1+\alpha(t)=\frac{1}{\|W\|_{\dot{H}^{1}}^{2}}\left(u_{[\theta(t), \mu(t)]}, W\right)_{\dot{H}^{1}} \text { and } \tilde{u}(t) \in H^{\perp} .
\end{aligned}
$$

We define $v(t)$ by

$$
v(t):=\alpha(t) W+\tilde{u}(t)=u_{[\theta(t), \mu(t)]}(t)-W .
$$

Recall that $\mu, \theta$ and $\alpha$ are $C^{1}$. If $a$ and $b$ are two positive quantities, we write $a \approx b$ when $C^{-1} a \leq b \leq C a$ with a positive constant $C$ independent of all parameters of the problem. We will prove the following lemma, which is a consequence of Claim 3.5 and of the equation satisfied by $v$, in Appendix 7.1 .

Lemma 3.7 (Modulation for threshold solutions of (1.1)). Taking a smaller $\delta_{0}$ if necessary, we have the following estimates on $I$.

$$
\begin{gathered}
|\alpha(t)| \approx\|v(t)\|_{\dot{H}^{1}} \approx\|\tilde{u}(t)\|_{\dot{H}^{1}} \approx \mathrm{d}(u(t)) \\
\left|\alpha^{\prime}(t)\right|+\left|\theta^{\prime}(t)\right|+\left|\frac{\mu^{\prime}(t)}{\mu(t)}\right| \leq C \mu^{2}(t) \mathrm{d}(u(t)) .
\end{gathered}
$$

Furthermore, $\alpha(t)$ and $\|u(t)\|_{\dot{H}^{1}}^{2}-\|W\|_{\dot{H}^{1}}^{2}$ have the same sign.

\subsection{Non-oscillatory behavior near $W_{\left[\theta_{0}, \mu_{0}\right]}$.}

Lemma 3.8. Let $\left(t_{0 n}\right)_{n}$ and $\left(t_{1 n}\right)_{n}, t_{0 n}<t_{1 n}$, be 2 real sequences, $\left(u_{n}\right)_{n}$ a sequence of radial solutions of (1.1) on $\left[t_{0 n}, t_{1 n}\right]$ such that $u_{n}\left(t_{1 n}\right)$ fullfills assumptions (3.1) and (3.2), and $\left(\lambda_{n}\right)_{n \in \mathbb{N}}$ a sequence of positive functions such that the set:

$$
\widetilde{K}=\left\{\left(u_{n}(t)\right)_{\left[\lambda_{n}(t)\right]}, n \in \mathbb{N}, t \in\left(t_{1 n}, t_{2 n}\right)\right\}
$$

is relatively compact in $\dot{H}^{1}$. Assume

$$
\lim _{n \rightarrow+\infty} \mathrm{d}\left(u_{n}\left(t_{0 n}\right)\right)+\mathrm{d}\left(u_{n}\left(t_{1 n}\right)\right)=0 .
$$

Then

$$
\lim _{n \rightarrow+\infty}\left\{\sup _{t \in\left(t_{0 n}, t_{1 n}\right)} \mathrm{d}\left(u_{n}(t)\right)\right\}=0 .
$$

Remark 3.9. Let $u$ be a solution of (1.1) satisfying the assumptions of Proposition 3.1, and $\lambda(t)$ the parameter given by Proposition 2.1. Let $\left(t_{n}\right)$ be a sequence, given by Corollary 3.4. such that $\mathrm{d}\left(u\left(t_{n}\right)\right)$ tends to 0 . Then the assumptions of the preceding proposition are fullfilled with $u_{n}=u, \lambda_{n}=\lambda, t_{0 n}=t_{n}, t_{1 n}=t_{n+1}$. 
Under the assumptions of Lemma 3.8, if $n$ is large enough so that $\mathrm{d}\left(u_{n}(t)\right)<\delta_{0}$ on the interval $\left(t_{0 n}, t_{1 n}\right)$, we will denote by $\theta_{n}(t), \mu_{n}(t)$ and $\alpha_{n}(t)$ the parameters of decomposition (3.21)

$$
\left(u_{n}(t)\right)_{\left[\theta_{n}(t), \mu_{n}(t)\right]}=\left(1+\alpha_{n}(t)\right) W+\tilde{u}_{n}(t) .
$$

Then we can complete Lemma 3.8 by the following.

Lemma 3.10. Under the assumptions of Lemma 3.8,

$$
\lim _{n \rightarrow+\infty} \frac{\sup _{t \in\left(t_{0 n}, t_{1 n}\right)} \mu_{n}(t)}{\inf _{t \in\left(t_{0 n}, t_{1 n}\right)} \mu_{n}(t)}=1
$$

Using the scaling invariance, it is sufficient to prove the preceding Lemmas assuming

$$
\forall n, \quad \inf _{t \in\left[t_{0 n}, t_{1 n}\right]} \lambda_{n}(t)=1 .
$$

Indeed, let $\ell_{n}:=\inf _{t \in\left(t_{0 n}, t_{1 n}\right)} \lambda_{n}(t)$, and

$$
\begin{gathered}
u_{n}^{*}(t, x)=\frac{1}{\ell_{n}^{\frac{N-2}{2}}} u_{n}\left(\frac{t}{\ell_{n}^{2}}, \frac{x}{\ell_{n}}\right), \quad \lambda_{n}^{*}(t)=\frac{\lambda_{n}(t)}{\ell_{n}}, \quad t_{0 n}^{*}=\frac{t_{0 n}}{\ell_{n}^{2}}, \quad t_{1 n}^{*}=\frac{t_{1 n}}{\ell_{n}^{2}} \\
\widetilde{K}^{*}=\left\{\left(u_{n}^{*}(t)\right)_{\left[\lambda_{n}^{*}(t)\right]}, n \in \mathbb{N}, t \in\left(t_{0 n}^{*}, t_{1 n}^{*}\right)\right\} .
\end{gathered}
$$

Then $u_{n}^{*}, t_{0 n}^{*}, t_{1 n}^{*}, \lambda_{n}^{*}$ and $\widetilde{K}^{*}$ fullfill the assumptions of Lemmas 3.8 and 3.10, Furthermore, the conclusions (3.25) and (3.27) of the Lemmas are not changed by the preceding transformations. We will thus assume (3.28) throughout the proofs.

The key point of the proofs is the following claim, which is a consequence of a localized virial argument.

Claim 3.11. Let $\left(u_{n}\right)_{n}$ be a sequence fullfilling the assumptions of Lemma 3.8 and (3.28). Then

$$
\forall n \in \mathbb{N}, \quad \int_{t_{0 n}}^{t_{1 n}} \mathrm{~d}\left(u_{n}(t)\right) d t \leq C\left[\mathrm{~d}\left(u_{n}\left(t_{0 n}\right)\right)+\mathrm{d}\left(u_{n}\left(t_{1 n}\right)\right)\right] .
$$

Before proving Claim 3.11, we will show that it implies the above lemmas.

Proof of Lemma 3.8. Let $\left(u_{n}\right)_{n}$ be as in Lemma 3.8, and assume (3.28). We first prove:

Claim 3.12. If $t_{n} \in\left(t_{0 n}, t_{1 n}\right)$ and the sequence $\lambda_{n}\left(t_{n}\right)$ is bounded, then

$$
\lim _{n \rightarrow+\infty} \mathrm{d}\left(u_{n}\left(t_{n}\right)\right)=0 .
$$

Proof. By our assumptions, $1 \leq \lambda_{n}\left(t_{n}\right) \leq C$, for some $C>1$, so that the sequence $u_{n}\left(t_{n}\right)$ is compact. Assume that (3.29) does not hold, so that, up to the extraction of a subsequence

$$
\lim _{n \rightarrow+\infty} u_{n}\left(t_{n}\right)=v^{0} \text { in } \dot{H}^{1}\left(\mathbb{R}^{N}\right), \quad \mathrm{d}\left(v^{0}\right)>0, E\left(v^{0}\right)=E(W) \text { and }\left\|v^{0}\right\|_{\dot{H}^{1}}<\|W\|_{\dot{H}^{1}} .
$$

Let $v$ be the solution of (1.1) with initial condition $v^{0}$ at time $t=0$, which is defined for $t \geq 0$. Note that for large $n, 1+t_{n} \leq t_{1 n}$. If not, $t_{1 n} \in\left(t_{n}, 1+t_{n}\right)$ for an infinite number of $n$, so that extracting a subsequence, $t_{1 n}-t_{n}$ has a limit $\tau \in[0,1]$. By the continuity of the flow of (1.1) in $\dot{H}^{1}, u_{n}\left(t_{1 n}\right)$ tends to $v(\tau)$ with $E(v(\tau))=E(W)$ and, by (3.24), $\mathrm{d}(v(\tau))=0$. This shows that 
$v=W_{\left[\theta_{0}, \lambda_{0}\right]}$ for some $\theta_{0}, \lambda_{0}$, contradicting (3.30). Thus $\left(t_{n}, 1+t_{n}\right) \subset\left(t_{0 n}, t_{1 n}\right)$. By (3.30) and the continuity of the flow of (1.1),

$$
\lim _{n \rightarrow+\infty} \int_{t_{n}}^{1+t_{n}} \mathrm{~d}\left(u_{n}(t)\right) d t=\int_{0}^{1} \mathrm{~d}(v(t)) d t>0 .
$$

Furthermore, according to Claim $3.11 \lim _{n} \int_{t_{0 n}}^{t_{1 n}} \mathrm{~d}\left(u_{n}(t)\right) d t=0$. which contradicts (3.31). The proof is complete.

By assumption (3.28), one may chose, for every $n, b_{n} \in\left(t_{0 n}, t_{1 n}\right)$ such that

$$
\lim _{n \rightarrow+\infty} \lambda_{n}\left(b_{n}\right)=1 \text {. }
$$

By Claim 3.12

$$
\lim _{n \rightarrow+\infty} \mathrm{d}\left(u_{n}\left(b_{n}\right)\right)=0 .
$$

We will show (3.25) by contradiction. Let us assume (after extraction) that for some $\delta_{1}>0$,

$$
\forall n, \quad \sup _{t \in\left(t_{0 n}, b_{n}\right)} \mathrm{d}\left(u_{n}(t)\right) \geq \delta_{1}>0
$$

(the proof is the same when $\left(t_{0 n}, b_{n}\right)$ is replaced by $\left(b_{n}, t_{1 n}\right)$ in the supremum). Fix $\delta_{2}>0$ smaller than $\delta_{1}$ and the constant $\delta_{0}$ given by Lemma 3.6. The mapping $t \mapsto \mathrm{d}\left(u_{n}(t)\right)$ being continuous, there exists $a_{n} \in\left(t_{0 n}, b_{n}\right)$ such that

$$
\mathrm{d}\left(u_{n}\left(a_{n}\right)\right)=\delta_{2} \text { and } \forall t \in\left(a_{n}, b_{n}\right), \mathrm{d}\left(u_{n}(t)\right)<\delta_{2} .
$$

On $\left(a_{n}, b_{n}\right)$, the modulation parameter $\mu_{n}$ is well defined. Furthermore, by the relative compactness of $\widetilde{K}$ and decomposition (3.26), the set $\bigcup_{n}\left\{W_{\left[\lambda_{n}\left(t_{n}\right) / \mu_{n}\left(t_{n}\right)\right.}(t), t \in\left[a_{n}, b_{n}\right]\right\}$ must be relatively compact, which shows

$$
\exists C>0, \forall t \in\left(a_{n}, b_{n}\right), \quad C^{-1} \lambda_{n}(t) \leq \mu_{n}(t) \leq C \lambda_{n}(t) .
$$

By (3.32), extracting a subsequence if necessary, we may assume

$$
\mu_{n}\left(b_{n}\right) \underset{n \rightarrow+\infty}{\longrightarrow} \mu_{\infty} \in(0, \infty) \text {. }
$$

Let us show by contradiction

$$
\sup _{n, t \in\left(a_{n}, b_{n}\right)} \mu_{n}(t)<\infty .
$$

If not, in view of the continuity of $\mu_{n}$, there exists (for large $\left.n\right) c_{n} \in\left(a_{n}, b_{n}\right)$ such that

$$
\mu_{n}\left(c_{n}\right)=2 \mu_{\infty}, \quad \mu_{n}(t)<2 \mu_{\infty}, t \in\left(c_{n}, b_{n}\right) .
$$

By Claim 3.12, $\lim _{n} \mathrm{~d}\left(u\left(c_{n}\right)\right)=0$. Furthermore, by Lemma $3.7,\left|\frac{\mu_{n}^{\prime}(t)}{\mu_{n}^{3}(t)}\right| \leq C \mathrm{~d}\left(u_{n}(t)\right)$. Integrating between $c_{n}$ and $b_{n}$, we get, by Claim 3.11 ,

$$
\left|\frac{1}{\mu_{n}^{2}\left(c_{n}\right)}-\frac{1}{\mu_{n}^{2}\left(b_{n}\right)}\right| \leq C \int_{c_{n}}^{b_{n}} \mathrm{~d}\left(u_{n}(s)\right) d s \underset{n \rightarrow+\infty}{\longrightarrow} 0,
$$

which contradicts the fact that $\mu_{n}\left(c_{n}\right)=2 \mu_{\infty}$ and $\mu_{n}\left(b_{n}\right) \rightarrow \mu_{\infty}$, and thus concludes the proof of (3.36).

By (3.36),$\mu_{n}\left(a_{n}\right)$ is bounded. Claim 3.12 shows that $\mathrm{d}\left(u_{n}\left(a_{n}\right)\right)$ tends to 0 , contradicting (3.34). The proof of Lemma 3.8 is complete. 
Proof of Lemma 3.10. It follows from the argument before Claim 3.11 that we may assume (3.28) in addition to the assumptions of the lemma, so that by (3.35)

$$
\exists C>0, \forall n, \quad C^{-1} \leq \inf _{t \in\left[t_{0 n}, t_{1 n}\right]} \mu_{n}(t) \leq C .
$$

Furthermore, in view of the continuity of $\mu_{n}$, there exist $a_{n}, b_{n} \in\left[t_{0 n}, t_{1 n}\right]$ such that

$$
\mu_{n}\left(a_{n}\right)=\inf _{t \in\left[t_{0 n}, t_{1 n}\right]} \mu_{n}(t), \quad \mu_{n}\left(b_{n}\right)=\sup _{t \in\left[t_{0 n}, t_{1 n}\right]} \mu_{n}(t) .
$$

By the bound $\left|\frac{\mu_{n}^{\prime}(t)}{\mu_{n}^{3}(t)}\right| \leq C \mathrm{~d}\left(u_{n}(t)\right)$, Claim 3.11, and Lemma 3.8, we get

$$
\lim _{n \rightarrow+\infty}\left|\frac{1}{\mu_{n}^{2}\left(a_{n}\right)}-\frac{1}{\mu_{n}^{2}\left(b_{n}\right)}\right|=0 .
$$

In particular, $\mu_{n}\left(b_{n}\right)$ is bounded. Multiplying the preceding limit by $\mu_{n}^{2}\left(b_{n}\right)$ yields (3.27).

Proof of Claim 3.11. Let us consider, for $R>0$, the function $G_{R, n}$ defined as in Subsection 3.1 by

$$
G_{R, n}(t)=2 \operatorname{Im} \int \bar{u}_{n}(t) \nabla u_{n}(t) \cdot \nabla \varphi_{R}
$$

$\left(\varphi_{R}\right.$ is defined in Subsection 3.1).

Step 1: a bound for $G_{R, n}$. In this step we show that there exists a constant $C>0$ such that

$$
\forall R>0, \forall n, \forall t \in\left(t_{0 n}, t_{1 n}\right), \quad\left|G_{R, n}(t)\right| \leq C R^{2} \mathrm{~d}\left(u_{n}(t)\right) .
$$

We have

$$
G_{R, n}(t)=2 \operatorname{Im} \int \bar{u}_{n}(t, x) \nabla u_{n}(t, x) \cdot R \nabla \varphi(x / R) d x .
$$

By Cauchy-Schwarz and Hardy inequalities $\left|G_{R, n}(t)\right| \leq R^{2}\left\|u_{n}\right\|_{\dot{H}^{1}}^{2}$, so that it suffices to show (3.39) when $\mathrm{d}\left(u_{n}(t)\right) \leq \delta_{1}$ for some small $\delta_{1}$. In this case, one may decompose $u_{n}$ as in (3.21), writing $\left(u_{n}(t)\right)_{\left[\theta_{n}(t), \mu_{n}(t)\right]}=W+v_{n}(t)$, with $\left\|v_{n}(t)\right\|_{\dot{H}^{1}} \leq C \mathrm{~d}\left(u_{n}(t)\right)$ by Lemma 3.8. By the change of variable $x=\frac{y}{\mu_{n}(t)}$,

$$
\begin{aligned}
G_{R, n}(t) & =2 \operatorname{Im} \frac{R}{\mu_{n}(t)} \int \frac{1}{\mu_{n}^{\frac{N-2}{2}}(t)} \bar{u}_{n}\left(t, \frac{y}{\mu_{n}(t)}\right) \frac{1}{\mu_{n}^{N / 2}(t)}\left(\nabla u_{n}\right)\left(t, \frac{y}{\mu_{n}(t)}\right) \cdot \nabla \varphi\left(\frac{y}{R \mu_{n}(t)}\right) d y \\
& =2 R^{2} \operatorname{Im} \int \frac{1}{R \mu_{n}(t)}\left(W+\bar{v}_{n}\right) \nabla\left(W+v_{n}\right) \cdot(\nabla \varphi)\left(\frac{y}{R \mu_{n}(t)}\right) d y .
\end{aligned}
$$

Write

$$
\operatorname{Im}\left[\left(W+\bar{v}_{n}\right) \nabla\left(W+v_{n}\right)\right]=\operatorname{Im}\left(W \nabla v_{n}+\bar{v}_{n} \nabla W+\bar{v}_{n} \nabla v_{n}\right),
$$

and note that on the support of $\nabla \varphi\left(\frac{y}{R \mu_{n}(t)}\right), \frac{1}{R \mu_{n}(t)}$ is bounded by $\frac{2}{|y|}$. As a consequence of Cauchy-Schwarz and Hardy inequalities, we get the bound $\left|G_{R, n}(t)\right| \leq C R^{2}\left(\left\|v_{n}(t)\right\|_{\dot{H}^{1}}+\right.$ $\left\|v_{n}(t)\right\|_{\dot{H}^{1}}^{2}$ ), which yields (3.39), for $\mathrm{d}\left(u_{n}(t)\right) \leq \delta_{1}, \delta_{1}$ small. The proof of (3.39) is complete. 
Step 2: a bound from below for $G_{R, n}^{\prime}$. The next and last step of the proof of Claim 3.11 is to show

$$
\exists R_{0}, \forall R \geq R_{0}, \forall n, \forall t \in\left(t_{0 n}, t_{1 n}\right), \quad G_{R, n}^{\prime}(t) \geq \frac{8}{N-2} \mathrm{~d}\left(u_{n}(t)\right) .
$$

It is clear that (3.39) and (3.40) imply the conclusion of Claim 3.11, Indeed, integrating (3.40) between $t_{0 n}$ and $t_{1 n}$ we get

$$
\frac{8}{N-2} \int_{t_{0 n}}^{t_{1 n}} \mathrm{~d}\left(u_{n}(t)\right) d t \leq G_{R_{0}, n}\left(t_{0 n}\right)+G_{R_{0}, n}\left(t_{1 n}\right),
$$

which shows the Claim in view of (3.39).

Let us show (3.40). Recall that by direct calculation we have, as in (3.9),

$$
G_{R, n}^{\prime}(t)=\frac{16}{N-2} \mathrm{~d}\left(u_{n}(t)\right)+A_{R}\left(u_{n}(t)\right),
$$

where $A_{R}$ is defined by (3.10). We first claim the following bounds on $A_{R}\left(u_{n}(t)\right)$ :

$$
\begin{aligned}
& \forall \varepsilon>0, \exists \rho_{\varepsilon}>0, \forall n, \forall t \in\left(t_{0 n}, t_{1 n}\right), \forall R \geq \frac{\rho_{\varepsilon}}{\lambda_{n}(t)}, \quad\left|A_{R}\left(u_{n}(t)\right)\right| \leq \varepsilon \\
& \exists \delta_{2}>0, \forall n, \forall t \in\left(t_{0 n}, t_{1 n}\right), \forall R \geq \frac{1}{\mu_{n}(t)}, \\
& \quad \mathrm{d}\left(u_{n}(t)\right) \leq \delta_{2} \Longrightarrow\left|A_{R, n}\left(u_{n}(t)\right)\right| \leq C\left(\frac{1}{\left(R \mu_{n}(t)\right)^{\frac{N-2}{2}}} \mathrm{~d}\left(u_{n}(t)\right)+\mathrm{d}\left(u_{n}(t)\right)^{2}\right) .
\end{aligned}
$$

The bound (3.42), follows directly from the compactness of $\widetilde{K}$, assumption (3.28), and the bound (3.12) of $A_{R}$ shown in the preceding section.

Let us show (3.43). Write as before

$$
\left(u_{n}(t)\right)_{\left[\theta_{n}(t), \mu_{n}(t)\right]}=W+v_{n}(t), \quad\left\|v_{n}(t)\right\|_{\dot{H}^{1}} \leq C \mathrm{~d}\left(u_{n}(t)\right) .
$$

In view of (3.44), estimate (3.43) is an immediate consequence of the existence of $\delta_{2}>0$ such that

$$
\begin{aligned}
& \forall g \in \dot{H}_{r}^{1}, \forall \mu_{0}>0, \forall R \geq \frac{1}{\mu_{0}}, \\
& \qquad\|g\|_{\dot{H}^{1}} \leq \delta_{2} \Longrightarrow\left|A_{R}\left((W+g)_{\left[\mu_{0}^{-1}\right]}\right)\right| \leq C\left(\frac{1}{\left(R \mu_{0}\right)^{\frac{N-2}{2}}}\|g\|_{\dot{H}^{1}}+\|g\|_{\dot{H}^{1}}^{2}\right)
\end{aligned}
$$

Let us show (3.45). A change of variable in $A_{R}$ gives $A_{R}\left((W+g)_{\left[\mu_{0}^{-1}\right]}\right)=A_{R \mu_{0}}(W+g)$. The function $W$ is a stationnary solution of (1.1), satisfying $\mathrm{d}(W)=0$ and $G_{R}(W)=0$, so that by (3.9), $A_{R}(W)=0$ for any $R>0$. Thus we must bound $A_{R \mu_{0}}(W+g)-A_{R \mu_{0}}(W)$. By the explicit form of $A_{R}$,

$$
A_{R}(f)=\int|\nabla f|^{2}\left(4 \frac{d^{2} \varphi_{R}}{d r^{2}}-8\right)+|f|^{2^{*}}\left(-\frac{4}{N} \Delta \varphi_{R}+8\right) r^{N-1} d r-\int|f|^{2}\left(\Delta^{2} \varphi_{R}\right) r^{N-1} d r
$$


and noting that the integrand in the first integral is supported in $\{|x| \geq R\}$ and in the second integral in $\{R \leq|x| \leq 2 R\}$, we get

$$
\begin{aligned}
\left|A_{R \mu_{0}}(W+g)-A_{R \mu_{0}}(W)\right| \leq C\left[\int_{|x| \geq R \mu_{0}}|\nabla g|^{2}\right. & +|\nabla W \cdot \nabla g|+W^{2^{*}-1}|g|+|g|^{2 *} d x \\
& \left.+\int_{R \mu_{0} \leq|x| \leq 2 R \mu_{0}} \frac{1}{\left(R \mu_{0}\right)^{2}}\left(W|g|+|g|^{2}\right) d x\right] .
\end{aligned}
$$

By explicit calculation, $\|\nabla W\|_{L^{2}(\{|x| \geq \rho\})} \approx\|W\|_{L^{2^{*}}(\{|x| \geq \rho\})} \approx \frac{1}{\rho^{\frac{N-2}{2}}}$ for large $\rho$. Hence, by Hardy, Sobolev and Cauchy-Schwarz inequalities

$$
\left|A_{R \mu_{0}}(W+g)-A_{R \mu_{0}}(W)\right| \leq C\left[\|g\|_{\dot{H}^{1}}^{2}+\|g\|_{\dot{H}^{1}}^{2 *}+\left(\frac{1}{\left(R \mu_{0}\right)^{\frac{N-2}{2}}}+\frac{1}{\left(R \mu_{0}\right)^{\frac{N+2}{2}}}\right)\|g\|_{\dot{H}^{1}}\right]
$$

which yields (3.45), and thus (3.43).

We are now ready to show (3.40). By assumption (3.28), $\lambda_{n}(t)$ is bounded from below. By (3.35), $\mu_{n}(t) \geq C_{*}>0$. Thus (3.43) implies for some $\delta_{3}>0, R_{1}>0$

$$
\mathrm{d}\left(u_{n}(t)\right) \leq \delta_{3}, R \geq R_{1} \Longrightarrow\left|A_{R}\left(u_{n}(t)\right)\right| \leq \frac{8}{N-2} \mathrm{~d}\left(u_{n}(t)\right) .
$$

Now, using (3.42) with $\varepsilon=\frac{8 \delta_{3}}{N-2}$ and again (3.28), we get $\left|A_{R}\left(u_{n}(t)\right)\right| \leq \frac{8}{N-2} \mathrm{~d}\left(u_{n}(t)\right)$ for $\mathrm{d}\left(u_{n}(t)\right) \geq \delta_{3}, R \geq R_{2}$. In view of (3.41), estimate (3.40) holds with $R_{0}:=\max \left\{R_{1}, R_{2}\right\}$, which concludes the proof of Claim 3.11 .

3.4. Proof of the convergence as $t$ goes to infinity. Let us show Proposition 3.1 and Corollary 3.2, Let $u$ be a radial solution of (1.1) satisfying (3.1) and (3.2).

Step 1: convergence of $\mathrm{d}(u(t))$ to 0 . We first prove (3.5). From Corollary 3.4, there exists a strictly increasing sequence $\left(t_{n}\right)_{n \in \mathbb{N}}$ such that:

$$
\lim _{n \rightarrow+\infty} t_{n}=+\infty, \quad \lim _{n \rightarrow+\infty} \mathrm{d}\left(u\left(t_{n}\right)\right)=0 .
$$

Let $t_{0 n}=t_{n}, t_{1 n}=t_{n+1}$, and $\lambda_{n}(t)=\lambda(t)$, where $\lambda$ is given by Proposition 2.1. Then the sequences $\left(u_{n}\right)_{n},\left(t_{0 n}\right)_{n},\left(t_{1 n}\right)_{n}$ and $\left(\lambda_{n}\right)_{n}$ clearly satisfy the assumptions of Lemma 3.8. Hence

$$
\lim _{n \rightarrow+\infty}\left(\sup _{t \in\left[t_{n}, t_{n+1}\right]} \mathrm{d}(u(t))\right)=0
$$

which clearly implies (3.5).

As a consequence of (3.5), we may decompose $u$ for large $t$ as in (3.21):

$$
u_{[\theta(t), \mu(t)]}=(1+\alpha(t)) W+\tilde{u}(t), \quad \tilde{u}(t) \in H^{\perp} .
$$

If $\theta, \mu$ and $\alpha$ are given by the preceding decomposition, the conclusion of Proposition 3.1 is equivalent to the existence of $\mu_{\infty}>0, \theta_{\infty} \in \mathbb{R}$ and $c, C>0$ such that

$$
\mathrm{d}(u(t))+|\alpha(t)|+\|\tilde{u}(t)\|_{\dot{H}^{1}}+\left|\theta(t)-\theta_{\infty}\right|+\left|\mu(t)-\mu_{\infty}\right| \leq C e^{-c t} .
$$


Step 2: convergence of $\mu$. We start to show by contradiction that $\mu(t)$ has a limit $\mu_{\infty} \in(0,+\infty)$ as $t \rightarrow+\infty$. If not, $\log (\mu(t))$ does not satisfy the Cauchy criterion as $t \rightarrow+\infty$, thus there exists sequences $T_{n}, T_{n}^{\prime} \rightarrow+\infty$ such that

$$
\lim _{n \rightarrow+\infty} \frac{\left|\mu\left(T_{n}\right)\right|}{\left|\mu\left(T_{n}^{\prime}\right)\right|}=L \neq 1
$$

Without loss of generality, we may assume $T_{n}<T_{n}^{\prime}$. By the preceding step, $d\left(u\left(T_{n}\right)\right)$ and $d\left(u\left(T_{n}^{\prime}\right)\right)$ tends to 0 . Let $u_{n}=u, t_{0 n}=T_{n}, t_{1 n}=T_{n}^{\prime}$, and $\lambda_{n}(t)=\lambda(t)$, where $\lambda$ is again given by Proposition 2.1. Then the assumptions of Lemma 3.10 are fullfilled, which shows

$$
\lim _{n \rightarrow+\infty} \frac{\inf _{T_{n} \leq t \leq T_{n}^{\prime}} \mu(t)}{\sup _{T_{n} \leq t \leq T_{n}^{\prime}} \mu(t)}=1 .
$$

This contradicts (3.47). Hence

$$
\lim _{t \rightarrow+\infty} \mu(t)=\mu_{\infty} \in(0, \infty) .
$$

Step 3: proof of Proposition 3.1. We are now ready to prove (3.46), which will complete the proof of Proposition 3.1. Let us first show that $\mathrm{d}(u(t))$ tends exponentially to 0 . We first claim the following inequality

$$
\exists C>0, \forall t \geq 0, \quad \int_{t}^{+\infty} \mathrm{d}(u(\tau)) d \tau \leq C \mathrm{~d}(u(t)) .
$$

Indeed if (3.49) does not hold, there exists a sequence $T_{n} \rightarrow+\infty$ such that

$$
\int_{T_{n}}^{+\infty} \mathrm{d}(u(\tau)) d \tau \geq n \mathrm{~d}\left(u\left(T_{n}\right)\right)
$$

By (3.48), $\mu(t)$ is bounded from below. As usual, this implies that the parameter $\lambda(t)$ of Proposition 2.1 is bounded from below. By Step 1 of the proof, the assumptions of Claim 3.11 are fullfilled for the sequence $\left(u_{k}\right)_{k}$, with $k=\left(n, n^{\prime}\right), n<n^{\prime}$, and $u_{k}=u, \lambda_{k}(t)=\lambda(t), t_{0 k}=T_{n}$ and $t_{1 k}=T_{n^{\prime}}$. Hence

$$
\forall n, n^{\prime}, n<n^{\prime}, \quad \int_{T_{n}}^{T_{n^{\prime}}} \mathrm{d}(u(t)) d t \leq C\left[\mathrm{~d}\left(u\left(T_{n}\right)\right)+\mathrm{d}\left(u\left(T_{n^{\prime}}\right)\right)\right],
$$

Thus $\int_{T_{n}}^{+\infty} \mathrm{d}(u(t)) d t \leq C \mathrm{~d}\left(u\left(T_{n}\right)\right)$ which contradicts (3.50), showing (3.49).

Now by (3.49) we have, for some constants $C, c>0$

$$
\int_{t}^{+\infty} \mathrm{d}(u(\tau)) d \tau \leq C e^{-c t}
$$

Together with the estimate $\left|\alpha^{\prime}(t)\right| \leq C \mathrm{~d}(u(t))$ of Lemma 3.7, we get

$$
|\alpha(t)|=\left|\int_{t}^{+\infty} \alpha^{\prime}(\tau) d \tau\right| \leq C e^{-c t}
$$

Recalling that by Lemma $3.7|\alpha(t)| \approx \mathrm{d}(u(t))$, we get the bound on $\mathrm{d}(u(t))$ in (3.46).

Estimate (3.46) is then a straightforward consequence of the estimate $\|\tilde{u}(t)\|_{\dot{H}^{1}}+\left|\theta^{\prime}(t)\right|+$ $\left|\frac{\mu^{\prime}(t)}{\mu(t)}\right| \leq C \mu^{2}(t) \mathrm{d}(u(t))$ of Lemma 3.7 and the boundedness of $\mu$. The proof of Proposition 3.1] is complete. 
Proof of Corollary 3.2. We must show that there is no solution $u$ of (1.1) satisfying (3.1) and (3.3). Let $u$ be such a solution. By Proposition 3.1 applied forward and backward, the set $\{u(t), t \in \mathbb{R}\}$, is relatively compact in $\dot{H}^{1}$. Furthermore

$$
\lim _{t \rightarrow+\infty} \mathrm{d}(u(t))=\lim _{t \rightarrow-\infty} \mathrm{d}(u(t))=0 .
$$

By Claim 3.11 with $u(t)=u_{n}(t), t_{0 n}=-n, t_{1 n}=n$ and $\lambda_{n}(t)=1$, we have $\int_{-\infty}^{+\infty} \mathrm{d}(u(t)) d t=$ $\lim _{n \rightarrow+\infty} \int_{-n}^{+n} \mathrm{~d}(u(t)) d t=0$. Thus $\mathrm{d}\left(u_{0}\right)=0$ which contradicts (3.1). Corollary 3.2] is proven.

\section{Convergence to $W$ in the supercritical Case}

In this section we consider a solution of (1.1) with initial condition $u_{\uparrow t=0}=u_{0}$ and such that

$$
E\left(u_{0}\right)=E(W), \quad\left\|u_{0}\right\|_{\dot{H}^{1}}>\|W\|_{\dot{H}^{1}} .
$$

Proposition 4.1. Let $u$ be a radial solution of (1.1) satisfying (4.1) and defined on $[0,+\infty)$. Assume furthermore that $u_{0} \in L^{2}\left(\mathbb{R}^{N}\right)$. Then there exist constants $\theta_{0} \in \mathbb{R}, \mu_{0}, c, C>0$ such that

$$
\forall t \geq 0, \quad\left\|u(t)-W_{\left[\theta_{0}, \mu_{0}\right]}\right\|_{\dot{H}^{1}} \leq C e^{-c t} .
$$

A similar result holds for negative times if $u$ satisfies (4.1) and is defined on $(-\infty, 0]$.

Corollary 4.2. Let $u$ be a radial solution of (1.1) satisfying (4.1) and such that $u_{0} \in L^{2}\left(\mathbb{R}^{N}\right)$. Then $u$ is not defined on $\mathbb{R}$.

The proof relies again on the localized virial argument. Consider a radial function $\varphi$ in $C_{0}^{\infty}\left(\mathbb{R}^{N}\right)$ such that

$$
\varphi(r)=r^{2}, r \leq 1, \quad \varphi(r) \geq 0 \text { and } \frac{d^{2} \varphi}{d r^{2}}(r) \leq 2, r \geq 0 .
$$

Consider the function $G_{R}$ of Subsection 3.1

$$
G_{R}(t):=2 \operatorname{Im} \int_{\mathbb{R}^{N}} \bar{u}(t) \nabla u(t) \cdot \nabla \varphi_{R}=H_{R}^{\prime}(t), \quad H_{R}(t):=\int_{\mathbb{R}^{N}}|u(t)|^{2} \varphi_{R} .
$$

where $\varphi_{R}(x)=R^{2} \varphi\left(\frac{x}{R}\right)$,

As usual, the key point of the proof is to bound $G_{R}$ and $G_{R}^{\prime}$.

Claim 4.3. Under the assumptions of Proposition 4.1, there exist constants $C, R_{0}>0$ (depending only on $\left.\int\left|u_{0}\right|^{2}\right)$, such that for $R \geq R_{0}$, and all $t \geq 0$

$$
\begin{aligned}
& G_{R}(t) \leq C R^{2} \mathrm{~d}(u(t)), \\
& G_{R}^{\prime}(t) \leq-\frac{8}{N-2} \mathrm{~d}(u(t)) .
\end{aligned}
$$

Let us show that Claim 4.3 implies Proposition 4.1.

Proof of Proposition 4.1. Step 1: exponential convergence of $\mathrm{d}(u(t))$. Let us prove

$$
\exists c, C>0, \forall t \geq 0, \quad \mathrm{~d}(u(t)) \leq C e^{-c t} .
$$

Fix $R \geq R_{0}$. We first remark

$$
\forall t \geq 0, \quad G_{R}(t)>0
$$


Indeed by (4.5), $G_{R}$ is strictly decreasing with time, so that if $G_{R}\left(t_{0}\right) \leq 0$ for some $t_{0} \geq 0$, then

$$
\forall t \geq t_{0}+1, \quad H_{R}^{\prime}(t)=G_{R}(t) \leq G_{R}\left(t_{0}+1\right)<0 .
$$

This contradicts the fact that $\varphi_{R}$ is positive and $u$ defined on $[0,+\infty)$, proving (4.7).

Consider two positive times $t<T$. Integrating (4.5) between $t$ and $T$, and using (4.4), we get

$$
\frac{8}{N-2} \int_{t}^{T} \mathrm{~d}(u(s)) d s \leq G_{R}(t)-G_{R}(T) \leq G_{R}(t) \leq C R^{2} \mathrm{~d}(u(t)) .
$$

Letting $T$ tends to infinity yields, $\int_{t}^{+\infty} \mathrm{d}(u(s)) d s \leq C \mathrm{~d}(u(t))$, for some $C>0$ and thus, by Gronwall Lemma

$$
\int_{t}^{+\infty} \mathrm{d}(u(s)) d s \leq C e^{-c t}
$$

Our next claim is that

$$
\lim _{t \rightarrow+\infty} \mathrm{d}(u(t))=0 .
$$

Indeed, by (4.9), there exists $t_{n} \rightarrow+\infty$ such that $\mathrm{d}\left(u\left(t_{n}\right)\right) \rightarrow 0$. Assume that (4.10) does not hold. Then, extracting a subsequence from $\left(t_{n}\right)$, there exists $t_{n}^{\prime}>t_{n}$ such that

$$
\mathrm{d}\left(u\left(t_{n}^{\prime}\right)\right)=\delta_{0}, \text { and } \forall t \in\left(t_{n}, t_{n}^{\prime}\right), 0<\mathrm{d}(u(t))<\delta_{0},
$$

where $\delta_{0}$ is such that (3.21) and Lemma 3.7 hold. Consider the parameter $\alpha$ of decomposition (3.21). By Lemma 3.7, $\left|\alpha^{\prime}(t)\right| \leq C \mathrm{~d}(u(t))$, for $t \in\left[t_{n}, t_{n}^{\prime}\right]$ thus (4.9) implies that $\alpha\left(t_{n}\right)-\alpha\left(t_{n}^{\prime}\right)$ tends to 0 . Furthermore, again by Lemma 3.7. $|\alpha(t)| \approx \mathrm{d}(u(t))$, which shows that $\mathrm{d}\left(u\left(t_{n}^{\prime}\right)\right)$ tends to 0 , contradicting the definition of $t_{n}^{\prime}$. Hence (4.10).

By (4.10), the parameter $\alpha(t)$ is well defined for large $t$. In view of the estimates $\left|\alpha^{\prime}(t)\right| \leq$ $C \mathrm{~d}(u(t))$ and $|\alpha(t)| \approx \mathrm{d}(u(t))$, (4.9) yields (4.6).

Step 2: convergence of $\mu(t)$ and end of the proof. Let us prove

$$
\lim _{t \rightarrow+\infty} \mu(t)=\mu_{\infty} \in(0, \infty) .
$$

By (4.6) and the estimate $\left|\frac{\mu^{\prime}(t)}{\mu^{3}(t)}\right| \leq C \mathrm{~d}(u(t))$ of Proposition 3.7, we know that $\frac{1}{\mu^{2}(t)}$ satisfies the Cauchy criterion of convergence. This shows that $\lim _{t \rightarrow+\infty} \mu(t)=\mu_{\infty} \in(0,+\infty]$. It remains to show that $\mu_{\infty}$ is finite.

Assume that $\mu_{\infty}=+\infty$. As $u_{[\theta(t), \mu(t)]}$ tends to $W$ in $\dot{H}^{1}$, it implies that for any $\varepsilon>0$, $\int_{|x| \geq \varepsilon}|u|^{2^{*}}$ tends to 0 as $t$ tends to $\infty$. By Hölder inequality and the boundedness of $\int|\nabla u(t)|^{2}$, this shows that $\lim _{t \rightarrow+\infty} H_{R}(t)=0$. Since by (4.7), $H_{R}^{\prime}(t)=G_{R}(t)>0$, this implies that $H_{R}(t)<0$ for $t \geq 0$ which contradicts the fact that $\varphi_{R}$ is positive. Hence (4.11).

In particular, $\mu$ is bounded. Thus by Lemma 3.7

$$
\left\|u-W_{[\theta(t), \mu(t)]}\right\|_{\dot{H}^{1}}+\left|\mu^{\prime}(t)\right|+\left|\theta^{\prime}(t)\right| \leq C \mathrm{~d}(u(t)) \leq C e^{-c t},
$$

which shows the Proposition.

Proof of Corollary 4.2. Let $u$ be a solution of (1.1) satisfying the assumptions of the corollary and defined on $\mathbb{R}$. Then by Proposition 4.1

$$
\lim _{t \rightarrow \pm \infty} \mathrm{d}(u(t))=0 .
$$


Define $G_{R}(t)$ as in the proof of Proposition 4.1. Applying Claim 4.3 to $t \mapsto \bar{u}(-t)$, we see that it holds also for negative times. By (4.5), $G_{R}^{\prime}(t)<0$ and by (4.4) and (4.12) $G_{R}(t) \rightarrow 0$ for $t \rightarrow \pm \infty$. This is a contradiction, yielding the corollary.

Proof of Claim 4.3. As in the proof of Lemma 3.3, since $E\left(u_{0}\right)=E(W)$ and $\left\|u_{0}\right\|_{\dot{H}^{1}}>\|W\|_{\dot{H}^{1}}$

$$
G_{R}^{\prime}(t)=8\left(\int|\nabla u|^{2}-\int|u|^{2 *}\right)+A_{R}(u(t))=-\frac{16}{N-2} \mathrm{~d}(u(t))+A_{R}(u(t)),
$$

where $A_{R}$ is defined in (3.10).

Step 1: a general bound on $A_{R}$. We show that there exist $C_{1}, R_{1}>0$ (depending only on $\int\left|u_{0}\right|^{2}$ ) such that

$$
\forall R \geq R_{1}, \forall t \geq 0, \quad A_{R}(u(t)) \leq\left\{\frac{C_{1}}{R^{2}}+\frac{C_{1}}{R^{\frac{2 N-2}{N-2}}}\|u(t)\|_{\dot{H}^{1}}^{\frac{2}{N-2}}\right\} .
$$

Indeed, according to (3.10), the definition of $\varphi_{R}$ and (4.3),

$$
\begin{aligned}
A_{R}(u(t))= & \int_{|x| \geq R}|\nabla u(t)|^{2}\left(4 \frac{d^{2} \varphi_{R}}{d r^{2}}-8\right) r^{N-1} d r \\
& +\int_{|x| \geq R}|u(t)|^{2^{*}}\left(-\frac{4}{N} \Delta \varphi_{R}+8\right) r^{N-1} d r-\int|u(t)|^{2}\left(\Delta^{2} \varphi_{R}\right) r^{N-1} d r \\
\leq & C \int_{|x| \geq R}|u(t)|^{2 *} d x+\frac{C}{R^{2}}\|u(t)\|_{L^{2}}^{2} .
\end{aligned}
$$

To bound the first term, we will use the radiality of $u(t)$ and Strauss Lemma [Str77a]:

Lemma 4.4. There is a constant $C>0$ such that for any radial function $f$ in $H^{1}\left(\mathbb{R}^{N}\right)$

$$
\forall x,|x| \geq 1, \quad|f(x)| \leq \frac{C}{|x|^{(N-1) / 2}}\|f\|_{L^{2}}^{1 / 2}\|f\|_{\dot{H}^{1}}^{1 / 2} .
$$

We have $\int_{|x| \geq R}|u(t)|^{2^{*}} \leq\|u(t)\|_{L^{\infty}(\{|x| \geq R\})}^{\frac{4}{N-2}}\|u(t)\|_{L^{2}}^{2}$, and thus, by Lemma 4.4,

$$
\int_{|x| \geq R}|u(t)|^{2^{*}} d x \leq \frac{C}{R^{\frac{2 N-2}{N-2}}}\|u(t)\|_{\dot{H}^{1}}^{\frac{2}{N-2}}\|u(t)\|_{L^{2}}^{\frac{2 N-2}{N-2}}
$$

which concludes the proof of (4.14) by the conservation of the $L^{2}$-norm.

Step 2: estimate on $A_{R}$ when $\mathrm{d}(u(t))$ is small. Let us show that there exists $\delta_{2}, R_{2}, C_{2}>0$ (depending only on $\int\left|u_{0}\right|_{L^{2}}^{2}$ ) such that

$$
\forall t \geq 0, \forall R \geq R_{2}, \quad \mathrm{~d}(u(t)) \leq \delta_{2} \Longrightarrow\left|A_{R}(u(t))\right| \leq C_{2}\left(\frac{1}{R^{\frac{N-2}{2}}} \mathrm{~d}(u(t))+\mathrm{d}(u(t))^{2}\right) .
$$

Taking a small $\delta_{2}$, we write by (3.21),$u_{[\theta(t), \mu(t)]}=W+v$, with $\|v\|_{\dot{H}^{1}} \leq C \mathrm{~d}(u(t))$. In view of the bound (3.45) of $A_{R}$ shown in the preceding section, it is sufficient to prove

$$
\mu_{-}:=\inf \left\{\mu(t), t \geq 0, \mathrm{~d}(u(t)) \leq \delta_{2}\right\}>0 .
$$


Inequality (4.17) follows again from the fact that $u_{0}$ is in $L^{2}$. Indeed, if $\mathrm{d}(u(t)) \leq \delta_{2}$ we have, from the conservation of the $L^{2}$ norm, the equality $u_{[\theta(t), \mu(t)]}=W+V$ and Lemma 3.7

$$
\left\|u_{0}\right\|_{L^{2}}^{2} \geq \int_{|x| \leq \mu(t)}|u(t)|^{2}=\frac{1}{\mu(t)^{2}} \int_{|x| \leq 1}\left|u_{[\mu(t)]}(t)\right|^{2} \geq \frac{1}{\mu(t)^{2}}\left[\left(\int_{|x| \leq 1} W^{2}\right)-C \delta_{2}^{2}\right] .
$$

If $\delta_{2}$ is small enough, this shows (4.17), and thus the announced inequality (4.16).

Step 3: conclusion of the proof. In view of (4.13), it is sufficient to prove

$$
\exists R_{0}>0, \forall R \geq R_{0}, \forall t \geq 0, \quad\left|A_{R}(u(t))\right| \leq \frac{8}{N-2} \mathrm{~d}(u(t)) .
$$

From (4.16), there exist $\delta_{3}, R_{3}$ such that (4.19) holds if $R \geq R_{3}$ and $\mathrm{d}(u(t)) \leq \delta_{3}$. Let $R_{4}>0$ and

$$
\Phi_{R_{4}}(\delta):=\frac{C_{1}}{R_{4}^{2}}+\frac{C_{1}}{R_{4}^{\frac{2 N-2}{N-2}}}\left(\delta+\|W\|_{\dot{H}^{1}}^{2}\right)^{\frac{1}{N-2}}-\frac{8}{N-2} \delta,
$$

where $C_{1}$ is given by step 1 . Clearly, $\Phi_{R_{4}}$ is concave. Chose $R_{4} \geq R_{2}$ large enough so that $\Phi_{R_{4}}\left(\delta_{3}\right) \leq 0, \Phi_{R_{4}}^{\prime}\left(\delta_{3}\right) \leq 0$. Then $\Phi_{R_{4}}(\delta) \leq 0$ for all $\delta \geq \delta_{3}$. Thus (4.14) implies (4.19) when $R \geq R_{4}$ and $\delta \geq \delta_{3}$, which concludes the proof of (4.19) with $R_{0}:=\max \left\{R_{3}, R_{4}\right\}$. The proof of Claim 4.3 is complete.

\section{Preliminaries on the linearized equation around $W$}

By Propositions 3.1 and 4.1, in order to conclude the proofs of Theorems 1 and 2, we need to study solutions $u$ of (1.1) on $\left[t_{0},+\infty\right),\left(t_{0} \geq 0\right)$ such that

$$
\|u(t)-W\|_{\dot{H}^{1}} \leq C e^{-\gamma_{0} t}, \quad E(u)=E(W)
$$

for some $\gamma_{0}>0$.

We will write indifferently $f=f_{1}+i f_{2}$ or $f=\left(\begin{array}{l}f_{1} \\ f_{2}\end{array}\right)$ for a complex valued function $f$ with real part $f_{1}$ and imaginary part $f_{2}$. For a solution $u$ of (1.1) satisfying (5.1), we will write

$$
v(t):=u(t)-W .
$$

Equation (1.1) yields

$$
\begin{gathered}
\partial_{t} v+\mathcal{L}(v)+R(v)=0, \quad \mathcal{L}:=\left(\begin{array}{cc}
0 & \Delta+W^{p_{c}-1} \\
-\Delta-p_{c} W^{p_{c}-1} & 0
\end{array}\right), \\
R(v):=-i|W+v|^{p_{c}-1}(W+v)+i W^{p_{c}}+i p_{c} W^{p_{c}-1} v_{1}-W^{p_{c}-1} v_{2} .
\end{gathered}
$$

The proofs of our theorems in Section 6 rely on a careful analysis of solutions of the linearized equation $\partial_{t} h+\mathcal{L} h=\varepsilon$, with $h$ and $\varepsilon$ exponentially small as $t \rightarrow+\infty$. Before this analysis, carried out in Subsection 5.3, we need to establish some spectral properties of $\mathcal{L}$ and Strichartz type estimates for the equation. 
5.1. Spectral theory for the linearized operator. We are interested here by real eigenvalues and other spectral properties of $\mathcal{L}$. Note that by direct calculation,

$$
\mathcal{L}(i W)=\mathcal{L}\left(W_{1}\right)=0 .
$$

Lemma 5.1. The operator $\mathcal{L}$ admits two eigenfunctions $\mathcal{Y}_{+}, \mathcal{Y}_{-} \in \mathcal{S}$ with real eigenvalues

$$
\mathcal{L} \mathcal{Y}_{+}=e_{0} \mathcal{Y}_{+}, \quad \mathcal{L} \mathcal{Y}_{-}=-e_{0} \mathcal{Y}_{-}, \quad \mathcal{Y}_{+}=\overline{\mathcal{Y}}_{-}, \quad e_{0} \in(0,+\infty)
$$

See Appendix 7.2 for the proof.

Consider the symmetric bilinear form $B$ on $\dot{H}^{1}$ such that $Q(f)=B(f, f)$, where $Q$ is the quadratic form of Subsection 3.2

$B(f, g)=\frac{1}{2} \int \nabla f_{1} \cdot \nabla g_{1}-\frac{p_{c}}{2} \int f_{1} g_{1} W^{p_{c}-1}+\frac{1}{2} \int \nabla f_{2} \cdot \nabla g_{2}-\frac{1}{2} \int f_{2} g_{2} W^{p_{c}-1}=\frac{1}{2} \operatorname{Im} \int(\mathcal{L} f) \bar{g}$.

As a consequence of the definition of $B$ we have,

$$
\begin{gathered}
B(f, g)=B(g, f), \quad B(i W, f)=B\left(W_{1}, f\right)=0, \quad \forall f, g \in \dot{H}^{1} \\
B(\mathcal{L} f, g)=-B(f, \mathcal{L} g), \quad \forall f, g \in \dot{H}^{1}, \mathcal{L} f, \mathcal{L} g \in \dot{H}^{1} \\
Q\left(\mathcal{Y}_{+}\right)=Q\left(\mathcal{Y}_{-}\right)=0, \quad B\left(\mathcal{Y}_{-}, \mathcal{Y}_{+}\right) \neq 0 .
\end{gathered}
$$

Indeed, the only assertion which is not direct is the fact that $B\left(\mathcal{Y}_{-}, \mathcal{Y}_{+}\right) \neq 0$. To prove it, one may argue by contradiction. If $B\left(\mathcal{Y}_{-}, \mathcal{Y}_{+}\right)$was $0, B$ and $Q$ would be identically 0 on $\operatorname{span}\left\{W_{1}, i W, \mathcal{Y}_{-}, \mathcal{Y}_{+}\right\}$which is of dimension 4 . But $Q$ is, by Claim 3.5. positive definite on $H^{\perp}$, which is of codimension 3, yielding a contradiction.

By (5.6), $\mathcal{L}$ is antisymmetric for the bilinear form $B$. In the following lemma, we give a subspace $G_{\perp}$ of $\dot{H}^{1}$, related to the eigenfunctions of $\mathcal{L}$, in which $Q$ is positive definite.

Lemma 5.2. Let $G_{\perp}=\left\{v \in \dot{H}^{1}, \quad(i W, v)_{\dot{H}^{1}}=\left(W_{1}, v\right)_{\dot{H}^{1}}=B\left(\mathcal{Y}_{+}, v\right)=B\left(\mathcal{Y}_{-}, v\right)=0\right\}$. Then there exists $c>0$ such that

$$
\forall f \in G_{\perp}, \quad Q(f) \geq c\|f\|_{\dot{H}^{1}}^{2} .
$$

Note that $G_{\perp}$ is not stable by $\mathcal{L}$. Lemma 5.2 implies the following characterization of the real spectrum of $\mathcal{L}$.

Corollary 5.3. Let $\sigma(\mathcal{L})$ be the spectrum of the operator $\mathcal{L}$ on $L^{2}$ of domain $D(\mathcal{L})=H^{2}$. Then

$$
\sigma(\mathcal{L}) \cap \mathbb{R}=\left\{-e_{0}, 0, e_{0}\right\}
$$

Proof of the corollary. By Lemma 5.1, $\left\{-e_{0}, e_{0}\right\} \subset \sigma(\mathcal{L})$. Furthermore, the operator $\mathcal{L}$ is a compact perturbation of $\left(\begin{array}{cc}0 & \Delta \\ -\Delta & 0\end{array}\right)$, thus its essential spectrum is $i \mathbb{R}$. Consequently, $0 \in \sigma(\mathcal{L})$, and $\sigma(\mathcal{L}) \cap \mathbb{R}^{*}$ contains only eigenvalues. It remains to show that $-e_{0}$ and $e_{0}$ are the only eigenvalues of $\mathcal{L}$ in $\mathbb{R}^{*}$. Assume that for some $f \in H^{2}$

$$
\mathcal{L} f=e_{1} f, \quad e_{1} \in \mathbb{R} \backslash\left\{-e_{0}, 0, e_{0}\right\} .
$$

We must show that $f=0$. By (5.6),$\left(e_{1}+e_{0}\right) B\left(f, \mathcal{Y}_{+}\right)=\left(e_{1}-e_{0}\right) B\left(f, \mathcal{Y}_{-}\right)=0$ and thus

$$
B\left(f, \mathcal{Y}_{+}\right)=B\left(f, \mathcal{Y}_{-}\right)=0 \text {. }
$$

Write

$$
f=\beta i W+\gamma W_{1}+g, \quad g \in G_{\perp}, \beta=\frac{(f, i W)_{\dot{H}^{1}}}{\|W\|_{\dot{H}^{1}}^{2}}, \gamma=\frac{\left(f, W_{1}\right)_{\dot{H}^{1}}}{\left\|W_{1}\right\|_{\dot{H}^{1}}^{2}} .
$$


Again by (5.6), $B(f, f)=0$ and thus $B(g, g)=0$. This implies by Lemma 5.2 that $g=0$ and thus $e_{1} f=\mathcal{L} f=\beta \mathcal{L}(i W)+\gamma \mathcal{L} W_{1}=0$. Recalling that $e_{1} \neq 0$, we get as announced that $f=0$, which concludes the proof of Corollary 5.3

Proof of Lemma 5.2. Recall from Claim 3.5 that there exists a constant $c_{1}$ such that

$$
\forall g \in H^{\perp}, \quad Q(g) \geq c_{1}\|g\|_{\dot{H}^{1}}^{2} .
$$

Let $f \in G_{\perp}$. We will eventually deduce (5.8) from (5.9). Decompose $f, \mathcal{Y}_{+}$and $\mathcal{Y}_{-}$in the orthogonal sum $\dot{H}^{1}=H \oplus H^{\perp}$ :

$$
f=\alpha W+\tilde{h}, \quad \mathcal{Y}_{+}=\eta i W+\xi W_{1}+\zeta W+h_{+}, \quad \mathcal{Y}_{-}=-\eta i W+\xi W_{1}+\zeta W+h_{-},
$$

where $\tilde{h}, h_{+}, h_{-} \in H^{\perp}, h_{-}=\bar{h}_{+}$.

Step 1. We first show

$$
Q(f)=-\frac{B\left(h_{+}, \tilde{h}\right) B\left(h_{-}, \tilde{h}\right)}{\sqrt{Q\left(h_{+}\right)} \sqrt{Q\left(h_{-}\right)}}+Q(\tilde{h}) .
$$

Note that if $h \in H^{\perp}, B(W, h)=\frac{1}{2} \int \nabla W \cdot \nabla h_{1}-\frac{p_{c}}{2} \int W^{p_{c}} h_{1}=\frac{1-p_{c}}{2} \int \nabla W \cdot \nabla h_{1}=0$. By (5.5), (5.7) and (5.10), we have

$$
0=Q\left(\mathcal{Y}_{+}\right)=\zeta^{2} Q(W)+Q\left(h_{+}\right), \quad 0=Q\left(\mathcal{Y}_{-}\right)=\zeta^{2} Q(W)+Q\left(h_{-}\right) .
$$

Furthermore, developping the equalities $B\left(f, \mathcal{Y}_{+}\right)=B\left(f, \mathcal{Y}_{-}\right)=0$ with (5.10) we get

$$
\alpha \zeta Q(W)+B\left(\tilde{h}, h_{+}\right)=\alpha \zeta Q(W)+B\left(\tilde{h}, h_{-}\right)=0 .
$$

By (5.10), $Q(f)=\alpha^{2} Q(W)+Q(\tilde{h})$, and (5.11) follows from (5.12) and (5.13).

Step 2. We next prove the following assertion:

The functions $h_{+}$and $h_{-}$are independent in the real Hilbert space $\dot{H}^{1}$.

Note that $h_{+}=\bar{h}_{-}$. Thus it is sufficient to show

$$
h_{1}:=\operatorname{Re} h \neq 0 \text { and } h_{2}:=\operatorname{Im} h \neq 0 .
$$

Write $\mathcal{Y}_{1}=\operatorname{Re} \mathcal{Y}_{+}, \mathcal{Y}_{2}=\operatorname{Im} \mathcal{Y}_{+}$. Then (5.4) writes down

$$
\left(\Delta+W^{p_{c}-1}\right) \mathcal{Y}_{2}=e_{0} \mathcal{Y}_{1}, \quad\left(\Delta+p_{c} W^{p_{c}-1}\right) \mathcal{Y}_{1}=-e_{0} \mathcal{Y}_{2}
$$

We show (5.14) by contradiction. First assume that $h_{2}=0$. Then by (5.10), $\mathcal{Y}_{2}$ is in $\operatorname{span}(W)$, so that $\left(\Delta+W^{p_{c}-1}\right) \mathcal{Y}_{2}=0$. By (5.15) , we get that $\mathcal{Y}_{1}=0$ and $\mathcal{Y}_{2}=0$, which contradicts the definition of $\mathcal{Y}_{+}$.

Similarly, assuming that $h_{1}=0$, and recalling that $\left(\Delta+p_{c} W^{p_{c}-1}\right) W_{1}=0$, we get by (5.10) and (5.15) that $\mathcal{Y}_{2}=-\frac{\zeta\left(p_{c}-1\right)}{e_{0}} W^{p_{c}}$, and thus $\mathcal{Y}_{1}=-\frac{\zeta\left(p_{c}-1\right)}{e_{0}^{2}}\left(\Delta+W^{p_{c}-1}\right) W^{p_{c}}$. Now, $\mathcal{Y}_{1}=$ $\xi W_{1}+\zeta W$. This implies that $\left(\Delta+W^{p_{c}-1}\right) W^{p_{c}}$ is in $\operatorname{span}\left\{W, W_{1}\right\}$, which is not the case as a direct computation shows. Hence (5.14) which concludes this step of the proof.

Step 3: conclusion of the proof. Recall that $Q$ is positive definite on $H^{\perp}$. We claim that there is a constant $b<1$, such that

$$
\forall X \in H^{\perp}, \quad\left|\frac{B\left(h_{+}, X\right) B\left(h_{-}, X\right)}{\sqrt{Q\left(h_{+}\right)} \sqrt{Q\left(h_{-}\right)}}\right| \leq b Q(X) .
$$


Indeed it is equivalent to show, by orthogonal decomposition on $H^{\perp}$ related to $B$

$$
b:=\max _{\substack{X \in \operatorname{span}\left\{h_{-}, h_{+}\right\} \\ X \neq 0}}\left(\frac{B\left(h_{+}, X\right)}{\sqrt{Q\left(h_{+}\right) Q(X)}}\right)\left(\frac{B\left(h_{-}, X\right)}{\sqrt{Q\left(h_{-}\right) Q(X)}}\right)<1 .
$$

Applying twice Cauchy-Schwarz inequality with $B$, we get $b \leq 1$. Furthermore, if $b=1$, there exists $X \neq 0$ such that the two Cauchy-Schwarz inequalities are equalities and thus $X \in \operatorname{span}\left\{h_{+}\right\} \cap \operatorname{span}\left\{h_{-}\right\}=\{0\}$, which is a contradiction, showing (5.16).

By (5.9), (5.11) and (5.16)

$$
Q(f) \geq(1-b) Q(\tilde{h}) \geq c_{1}(1-b)\|\tilde{h}\|_{\dot{H}^{1}}^{2} .
$$

Noting that by (5.10), $Q(f)=\alpha^{2} Q(W)+Q(\tilde{h})$, and recalling that $Q(W)<0$, we also get by the first inequality in (5.18) that $b Q(\tilde{h}) \geq \alpha^{2}|Q(W)|$. Hence

$$
C Q(f) \geq \alpha^{2}\|W\|_{\dot{H}^{1}}^{2}+\|\tilde{h}\|_{\dot{H}^{1}}^{2}=\|f\|_{\dot{H}^{1}}^{2},
$$

which concludes the proof of Lemma 5.2 .

5.2. Preliminary estimates. In this subsection we gather some elementary estimates needed in the sequel. We start with bounds on the potential and nonlinear terms of equation (5.2). Let

$$
\mathcal{V}(v):=W^{p_{c}-1} \operatorname{Re} v+p W^{p_{c}-1} \operatorname{Im} v,
$$

so that equation (5.2) writes as a Schrödinger equation

$$
i \partial_{t} v+\Delta v+\mathcal{V}(v)+i R(v)=0
$$

We start to recall standard Strichartz estimates for the free Schrödinger equation (see [Str77b, GV85, KT98]).

Lemma 5.4. Assume $N \geq 3$ and, for $j=1,2$, let $\left(p_{j}, q_{j}\right)$ such that $\left(\frac{2}{p_{j}}+\frac{N}{q_{j}}=\frac{N}{2}, p_{j} \geq 2\right)$. Denote by $p_{j}^{\prime}$ and $q_{j}^{\prime}$ the dual conjugate exponents of $p_{j}$ and $q_{j}$. Then

$$
\begin{aligned}
\left\|e^{i t \Delta} u_{0}\right\|_{L^{p_{1}\left(\mathbb{R}, L^{q_{1}}\right)}} & \leq C\left\|u_{0}\right\|_{L^{2}} \\
\left\|\int_{t}^{+\infty} e^{i(s-t) \Delta} f(s) d s\right\|_{L^{p_{1}\left(\mathbb{R}, L^{\left.q_{1}\right)}\right.}} & \leq C\|f\|_{L^{p_{2}^{\prime}\left(\mathbb{R}, L^{q_{2}^{\prime}}\right)}} \\
\left\|\int_{-\infty}^{+\infty} e^{i s \Delta} f(s) d s\right\|_{L^{2}} & \leq C\|f\|_{L^{p_{2}^{\prime}}}{\left.\mathbb{R}, L^{q_{2}^{\prime}}\right)}^{+}
\end{aligned}
$$

If $I$ is a time interval, we will be interested in the spaces $S(I)$ and $Z(I)$ defined in (2.4) as well as $N(I):=L^{2}\left(I ; L^{\frac{2 N}{N+2}}\right)$, which is the dual of the endpoint Strichartz space $L^{2}\left(I ; L^{2^{*}}\right)$. Hölder and Sobolev inequalities yield immediately:

Lemma 5.5 (Linear estimates). Let $f \in L^{2^{*}}\left(\mathbb{R}^{N}\right)$. Then

$$
\|\mathcal{V}(f)\|_{L^{\frac{2 N}{N+2}}} \leq C\|f\|_{L^{2^{*}}}
$$

Let $I$ be a finite time interval of length $|I|$ and $f \in S(I)$ such that $\nabla f \in Z(I)$. Then, there exists $C$ independent of $I, f$ and $g$ such that

$$
\begin{aligned}
\|f\|_{S(I)} & \leq C\|\nabla f\|_{Z(I)} \\
\|\nabla \mathcal{V}(f)\|_{N(I)} & \leq|I|^{\frac{N}{N+2}}\|\nabla f\|_{Z(I)} .
\end{aligned}
$$


The proof of following lemma, given in the appendix, is classical.

Lemma 5.6 (Non-linear estimates). Let $f, g$ be functions in $L^{2^{*}}\left(\mathbb{R}^{N}\right)$. Then

$$
\|R(f)-R(g)\|_{L^{\frac{2 N}{N+2}}} \leq C\|f-g\|_{L^{2^{*}}}\left(\|f\|_{L^{2^{*}}}+\|g\|_{L^{2^{*}}}+\|f\|_{L^{2^{*}}}^{p_{c}}+\|g\|_{L^{2^{*}}}^{p_{c}-1}\right) .
$$

Let $I$ be a finite time interval and $f, g$ be functions in $S(I)$, such that $\nabla f$ and $\nabla g$ are in $Z(I)$. Then

$$
\begin{aligned}
& \|\nabla R(f)-\nabla R(g)\|_{N(I)} \leq \\
& \qquad C\|\nabla f-\nabla g\|_{Z(I)}\left[|I|^{\frac{6-N}{2(N+2)}}\left(\|\nabla f\|_{Z(I)}+\|\nabla g\|_{Z(I)}\right)+\|\nabla f\|_{Z(I)}^{p_{c}-1}+\|\nabla g\|_{Z(I)}^{p_{c}-1}\right] .
\end{aligned}
$$

We finish this subsection by showing Strichartz estimates on exponentially small solutions $v$ of (5.2).

Lemma 5.7 (Strichartz estimates). Let $v$ be a solution of (5.2). Assume for some $c_{0}>0$,

$$
\exists C>0, \quad\|v(t)\|_{\dot{H}^{1}} \leq C e^{-c_{0} t} .
$$

Then, for any Strichartz couple $(p, q)\left(\frac{2}{p}+\frac{N}{q}=\frac{N}{2}, p \geq 2\right)$

$$
\exists C>0, \quad\|v\|_{S(t,+\infty)}+\|\nabla v\|_{L^{p}\left(t,+\infty ; L^{q}\right)} \leq C e^{-c_{0} t} .
$$

Proof. We will first estimate $\|v\|_{S(t,+\infty)}+\|\nabla v\|_{Z(t,+\infty)}$. According to the following claim, we juste need to estimate $\|v\|_{S\left(t, t+\tau_{0}\right)}$ and $\|\nabla v\|_{Z\left(t, t+\tau_{0}\right)}$ for some small $\tau_{0}>0$.

Claim 5.8 (Sums of exponential). Let $t_{0}>0, p \in\left[1,+\infty\left[, a_{0} \neq 0, E\right.\right.$ a normed vector space, and $f \in L_{\mathrm{loc}}^{p}\left(t_{0},+\infty ; E\right)$ such that

$$
\exists \tau_{0}>0, \exists C_{0}>0, \forall t \geq t_{0}, \quad\|f\|_{L^{p}\left(t, t+\tau_{0}, E\right)} \leq C_{0} e^{a_{0} t} .
$$

Then for $t \geq t_{0}$,

$$
\|f\|_{L^{p}(t,+\infty, E)} \leq \frac{C_{0} e^{a_{0} t}}{1-e^{a_{0} \tau_{0}}} \text { if } a_{0}<0 ; \quad\|f\|_{L^{p}\left(t_{0}, t, E\right)} \leq \frac{C_{0} e^{a_{0} t}}{1-e^{-a_{0} \tau_{0}}} \text { if } a_{0}>0 .
$$

Proof. Assume $a_{0}<0$. Summing up (5.31) at time $t=t_{0}, t=t_{0}+\tau_{0}, t=t_{0}+2 \tau_{0}, \ldots$, and using the triangle inequality, we get (5.32). The case $a_{0}>0$ is analogue.

By (5.20),

$$
i \partial_{t} \nabla v+\Delta(\nabla v)+\nabla(\mathcal{V}(v)+i R(v))=0 .
$$

Let $t$ and $\tau$ such that $0<t, 0<\tau<1$. By Strichartz inequalities (5.21) and (5.22), and equation (5.33)

$$
\|\nabla v\|_{Z(t, t+\tau)} \leq C\left(\|v(t)\|_{\dot{H}^{1}}+\|\nabla(\mathcal{V}(v)+R(v))\|_{N(t, t+\tau)}\right) .
$$

Thus by Lemmas 5.5 and 5.6

$$
\|\nabla v\|_{Z(t, t+\tau)} \leq C\left(\|v(t)\|_{\dot{H}^{1}}+\tau^{\frac{N}{N+2}}\|\nabla v\|_{Z(t, t+\tau)}+\tau^{\frac{6-N}{2(N+2)}}\|\nabla v\|_{Z(t, t+\tau)}^{2}+\|\nabla v\|_{Z(t, t+\tau)}^{p_{c}}\right) .
$$

Using assumption (5.29), we get, for some constants $K>0$ and $\alpha_{N}>0$

$$
\|\nabla v\|_{Z(t, t+\tau)} \leq K\left\{e^{-c_{0} t}+\tau^{\alpha_{N}}\|\nabla v\|_{Z(t, t+\tau)}+\|\nabla v\|_{Z(t, t+\tau)}^{p_{c}}\right\} .
$$


We claim that it implies that for large $t$

$$
\|\nabla v\|_{Z\left(t, t+\tau_{0}\right)} \leq 2 K e^{-c_{0} t}, \quad \tau_{0}:=\frac{1}{(3 K)^{1 / \alpha_{N}}} .
$$

Indeed, fix $t>0$. Then (5.34) implies $\|\nabla v\|_{Z(t, t+\tau)}<2 K e^{-c_{0} t}$ for small $\tau$. If (5.35) does not hold, then there exists $\tau \in\left(0, \tau_{0}\right]$ such that $\|\nabla v\|_{Z(t, t+\tau)}=2 K e^{-c_{0} t}$, contradicting (5.34) if $t$ is large. Hence (5.35).

By Claim 5.8 and Sobolev inequality (5.25)

$$
\|v\|_{S(t,+\infty)}+\|\nabla v\|_{Z(t,+\infty)} \leq C e^{-c_{0} t}
$$

Now take any Strichartz couple $(p, q)$. Then by (5.33), Strichartz estimates (5.21) and (5.22) and Lemmas 5.5 and 5.6, $\|\nabla v\|_{L^{p}\left(t, t+1 ; L^{q}\right)} \leq C\left(\|v(t)\|_{\dot{H}^{1}}+\|\nabla v\|_{Z(t, t+1)}+\|\nabla v\|_{Z(t, t+1)}^{p_{c}}\right)$. Thus (5.36) implies the bound $\|\nabla v\|_{L^{p}\left(t, t+1 ; L^{q}\right)} \leq C e^{-c_{0} t}$, which concludes, by Claim [5.8, the proof of the lemma.

5.3. Estimates on exponential solutions of the linearized equation. Let us consider the linearized equation with right-member

$$
\partial_{t} h+\mathcal{L} h=\varepsilon
$$

with $h$ and $\varepsilon$ such that for $t \geq 0$,

$$
\begin{gathered}
\|\nabla \varepsilon\|_{N(t,+\infty)}+\|\varepsilon(t)\|_{L^{2 N}} \leq C e^{-c_{1} t}, \\
\|h(t)\|_{\dot{H}^{1}} \leq C e^{-c_{0} t},
\end{gathered}
$$

where $0<c_{0}<c_{1}$. The following proposition asserts that $h$ must decay almost as fast as $\varepsilon$, except in the direction $\mathcal{Y}_{+}$where the decay is of order $e^{-e_{0} t}$.

Proposition 5.9. Consider $h$ and $\varepsilon$ satisfying (5.37), (5.38) and (5.39). Then, for any Strichartz couple $(p, q)$ :

- if $c_{0}<c_{1} \leq e_{0}$ or $e_{0}<c_{0}<c_{1}$,

$$
\forall \eta>0, \quad\|h(t)\|_{\dot{H}^{1}}+\|\nabla h\|_{L^{p}\left(t,+\infty ; L^{q}\right)} \leq C_{\eta} e^{-\left(c_{1}-\eta\right) t} ;
$$

- if $c_{0} \leq e_{0}<c_{1}$, there exists $A_{+} \in \mathbb{R}$ such that

$$
\forall \eta>0, \quad\left\|h(t)-A_{+} e^{-e_{0} t} \mathcal{Y}_{+}\right\|_{\dot{H}^{1}}+\left\|\nabla\left(h-A_{+} e^{-e_{0} t} \mathcal{Y}_{+}\right)\right\|_{L^{p}\left(t,+\infty ; L^{q}\right)} \leq C_{\eta} e^{-\left(c_{1}-\eta\right) t} .
$$

Proof of Proposition 5.9. We will start by proving (5.40). In view of the following claim it is sufficient to prove only the bound of the $\dot{H}^{1}$-norm.

Claim 5.10. Consider $h$ and $\varepsilon$ fullfilling (5.37), (5.38) and (5.39) with $0<c_{0}<c_{1}$. Then for any Strichartz couple $(p, q)$

$$
\|\nabla h\|_{L^{p}\left(t,+\infty ; L^{q}\right)} \leq C e^{-c_{0} t} .
$$

We will omit the proof, which is a simple consequence of Strichartz inequalities and of Lemma 5.5. and is similar to the proof of Lemma 5.7.

Let us decompose $h(t)$ as

$$
h(t)=\alpha_{+}(t) \mathcal{Y}_{+}+\alpha_{-}(t) \mathcal{Y}_{-}+\beta(t) i W+\gamma(t) W_{1}+g(t), \quad g(t) \in G_{\perp},
$$


where (recall that by (5.5) and (5.7), $B(i W, \cdot)=B\left(W_{1}, \cdot\right)=0$ and $\left.Q\left(\mathcal{Y}_{+}\right)=Q\left(\mathcal{Y}_{-}\right)=0\right)$

$$
\begin{gathered}
\alpha_{-}:=\frac{B\left(h, \mathcal{Y}_{+}\right)}{B\left(\mathcal{Y}_{+}, \mathcal{Y}_{-}\right)}, \quad \alpha_{+}:=\frac{B\left(h, \mathcal{Y}_{-}\right)}{B\left(\mathcal{Y}_{+}, \mathcal{Y}_{-}\right)} \\
\beta:=\frac{1}{\|W\|_{\dot{H}^{1}}^{2}}\left(h-\alpha_{+} \mathcal{Y}_{+}-\alpha_{-} \mathcal{Y}_{-}, i W\right)_{\dot{H}^{1}}, \quad \gamma:=\frac{1}{\left\|W_{1}\right\|_{\dot{H}^{1}}^{2}}\left(h-\alpha_{+} \mathcal{Y}_{+}-\alpha_{-} \mathcal{Y}_{-}, W_{1}\right)_{\dot{H}^{1}} .
\end{gathered}
$$

In the sequel, we will assume without loss of generality

$$
c_{1} \neq e_{0} .
$$

We divide the proof into four steps.

Step 1: differential equations on the coefficients. We first claim

$$
\begin{gathered}
\frac{d}{d t}\left(e^{e_{0} t} \alpha_{+}\right)=e^{e_{0} t} \frac{B\left(\mathcal{Y}_{-}, \varepsilon\right)}{B\left(\mathcal{Y}_{+}, \mathcal{Y}_{-}\right)}, \quad \frac{d}{d t}\left(e^{-e_{0} t} \alpha_{-}\right)=e^{-e_{0} t} \frac{B\left(\mathcal{Y}_{+}, \varepsilon\right)}{B\left(\mathcal{Y}_{+}, \mathcal{Y}_{-}\right)}, \\
\frac{d Q(h)}{d t}=2 B(h, \varepsilon), \quad \frac{d \beta}{d t}=\frac{(i W, \widetilde{\varepsilon})_{\dot{H}^{1}}}{\|W\|_{\dot{H}^{1}}^{2}}, \quad \frac{d \gamma}{d t}=\frac{\left(W_{1}, \widetilde{\varepsilon}\right)_{\dot{H}^{1}}}{\left\|W_{1}\right\|_{\dot{H}^{1}}^{2}} .
\end{gathered}
$$

Where

$$
\widetilde{\varepsilon}:=\varepsilon-\frac{B\left(\mathcal{Y}_{-}, \varepsilon\right)}{B\left(\mathcal{Y}_{+}, \mathcal{Y}_{-}\right)} \mathcal{Y}_{+}-\frac{B\left(\mathcal{Y}_{+}, \varepsilon\right)}{B\left(\mathcal{Y}_{+}, \mathcal{Y}_{-}\right)} \mathcal{Y}_{-}-\mathcal{L} g
$$

By equation (5.37),

$$
B\left(\mathcal{Y}_{-}, \partial_{t} h\right)+B\left(\mathcal{Y}_{-}, \mathcal{L} h\right)=B\left(\mathcal{Y}_{-}, \varepsilon\right) .
$$

Furthermore $B\left(\mathcal{Y}_{-}, \partial_{t} h\right)=\frac{d}{d t} B\left(\mathcal{Y}_{-}, h\right)$ and by (5.6),$B\left(\mathcal{Y}_{-}, \mathcal{L} h\right)=-B\left(\mathcal{L} \mathcal{Y}_{-}, h\right)=e_{0} B\left(\mathcal{Y}_{-}, h\right)$. In view of (5.44), we get the first equation in (5.47). A similar calculation yields the second equation.

By equation (5.37), $B\left(h, \partial_{t} h\right)+B(h, \mathcal{L} h)=B(h, \varepsilon)$. Furthermore by (5.6), $B(h, \mathcal{L} h)=0$ which yields the equation on $Q(h)$ in (5.48).

It remains to show the equations on $\beta$ and $\gamma$. Differentiating (5.45), we get

$$
\beta^{\prime}(t)=\frac{1}{\|W\|_{\dot{H}^{1}}^{2}}(\widehat{\varepsilon}, i W)_{\dot{H}^{1}}, \quad \gamma^{\prime}(t)=\frac{1}{\left\|W_{1}\right\|_{\dot{H}^{1}}^{2}}\left(\widehat{\varepsilon}, W_{1}\right)_{\dot{H}^{1}}, \quad \widehat{\varepsilon}:=\varepsilon-\mathcal{L} h-\alpha_{+}^{\prime} \mathcal{Y}_{+}-\alpha_{-}^{\prime} \mathcal{Y}_{-} .
$$

Now, noting that by (5.43), $\mathcal{L} h=\alpha_{+} e_{0} \mathcal{Y}_{+}-\alpha_{-} e_{0} \mathcal{Y}_{-}+\mathcal{L}(g)$, and using (5.47), we obtain

$$
\widehat{\varepsilon}=\varepsilon-\frac{B\left(\mathcal{Y}_{-}, \varepsilon\right)}{B\left(\mathcal{Y}_{+}, \mathcal{Y}_{-}\right)} \mathcal{Y}_{+}-\frac{B\left(\mathcal{Y}_{+}, \varepsilon\right)}{B\left(\mathcal{Y}_{+}, \mathcal{Y}_{-}\right)} \mathcal{Y}_{-}-\mathcal{L}(g)=\widetilde{\varepsilon}
$$

which yields the desired result.

Step 2: bounds on $\alpha_{-}$and $\alpha_{+}$. We now claim

$$
\begin{aligned}
& \left|\alpha_{-}(t)\right| \leq C e^{-c_{1} t} \\
& \left|\alpha_{+}(t)\right| \leq \begin{cases}C e^{-c_{1} t} & \text { if } e_{0}<c_{0}, \\
C\left(e^{-e_{0} t}+e^{-c_{1} t}\right) & \text { if } c_{0} \leq e_{0} .\end{cases}
\end{aligned}
$$

Let us first show the following general bound on $B$. 
Claim 5.11. For any finite time-interval $I$, of length $|I|$, and any functions $f$ and $g$ such that $f \in L^{\infty}\left(I, L^{\frac{2 N}{N+2}}\right), \nabla f \in N(I), g \in L^{\infty}\left(I, L^{2^{*}}\right)$ and $\nabla g \in L^{2}\left(I, L^{2^{*}}\right)$,

$$
\int_{I}|B(f(t), g(t))| d t \leq C\left[\|\nabla f\|_{N(I)}\|\nabla g\|_{L^{2}\left(I, L^{2 *}\right)}+|I|\|f\|_{L^{\infty}\left(I, L^{\frac{2 N}{N+2}}\right)}\|g\|_{L^{\infty}\left(I, L^{2^{*}}\right)}\right] .
$$

Proof. We have

$$
\begin{aligned}
2 B(f(t), g(t))=a(t)+b(t), \text { where } a(t) & :=\int_{\mathbb{R}^{N}} \nabla f_{1}(t) \nabla g_{1}(t)+\int_{\mathbb{R}^{N}} \nabla f_{2}(t) \nabla g_{2}(t) \\
b(t) & :=-p_{c} \int_{\mathbb{R}^{N}} W^{p_{c}-1} f_{1}(t) g_{1}(t)-\int_{\mathbb{R}^{N}} W^{p_{c}-1} f_{2}(t) g_{2}(t) .
\end{aligned}
$$

By Hölder inequality

$$
\int_{I}|a(t)| d t \leq C\|\nabla f\|_{N(I)}\|\nabla g\|_{L^{2}\left(I, L^{2^{*}}\right)}, \quad|b(t)| \leq C\|f(t)\|_{L^{\frac{2 N}{N+2}}}\|g(t)\|_{L^{2^{*}}}\left\|W^{p_{c}-1}\right\|_{L^{\infty}} .
$$

Integrating the estimate on $b(t)$ over $I$ and summing up, we get the conclusion of the claim.

Assumption (5.38) on $\varepsilon$, together with the preceding claim yields the inequality

$$
\int_{t}^{t+1}\left|e^{-e_{0} s} B\left(\mathcal{Y}_{+}, \varepsilon(s)\right)\right| d s \leq C e^{-\left(e_{0}+c_{1}\right) t} .
$$

By Claim 5.8, $\int_{t}^{\infty}\left|e^{-e_{0} s} B\left(\mathcal{Y}_{+}, \varepsilon(s)\right)\right| d s \leq C e^{-\left(e_{0}+c_{1}\right) t}$. Integrating the equation on $\alpha_{-}$in (5.47) between $t$ and $+\infty$, we get (5.50).

Let us show (5.51). First assume that $c_{0}>e_{0}$. Thus by assumption (5.39), $e^{e_{0} t} \alpha_{+}(t)$ tends to 0 when $t$ tends to infinity. Furthermore $c_{1}>c_{0}>e_{0}$, and thus by assumption (5.38), Claims 5.11 and 5.8, $\int_{t}^{+\infty}\left|e^{e_{0} s} B\left(\mathcal{Y}_{-}, \varepsilon(s)\right)\right| d s \leq C e^{\left(e_{0}-c_{1}\right) t}$. Integrating between $t$ and $+\infty$ the equation on $\alpha_{+}$in (5.47) we get (5.51) if $c_{0}>e_{0}$.

Now assume that $c_{0} \leq e_{0}$. By (5.47)

$$
\alpha_{+}(t)=e^{-e_{0} t} \alpha_{+}(0)+\frac{e^{-e_{0} t}}{B\left(\mathcal{Y}_{+}, \mathcal{Y}_{-}\right)} \underbrace{\int_{0}^{t} e^{e_{0} s} B\left(\mathcal{Y}_{-}, \varepsilon(s)\right) d s}_{(a)} .
$$

If $c_{0} \leq e_{0}<c_{1}$, assumption (5.38) and Claim 5.11 imply that the integral $(a)$ is bounded, which shows (5.51) in this case.

It remains to show (5.51) when $c_{0}<c_{1}<e_{0}$. By (5.38), Claim 5.11 and Claim 5.8, $|(a)| \leq$ $C e^{\left(e_{0}-c_{1}\right) t}$, which yields again (5.51). Step 2 is complete.

Step 3: bounds on $\|g\|_{\dot{H}^{1}}, \beta$ and $\gamma$. We next prove

$$
\|g(t)\|_{\dot{H}^{1}}+|\beta(t)|+|\gamma(t)| \leq C e^{-\left(\frac{c_{0}+c_{1}}{2}\right) t} .
$$

By Claims 5.10 and 5.11, assumptions (5.38) and (5.39) yield $\int_{t}^{t+1}|B(h(s), \varepsilon(s))| d s \leq C e^{-\left(c_{0}+c_{1}\right) t}$. Integrating the equation on $Q$ in (5.48) between $t$ and $+\infty$ and using Claim 5.8, we get

$$
|Q(h(t))| \leq C e^{-\left(c_{0}+c_{1}\right) t} .
$$


Thus

$$
\begin{gathered}
\left|Q\left(\alpha_{+} \mathcal{Y}_{+}+\alpha_{-} \mathcal{Y}_{-}+\beta i W+\gamma W_{1}+g\right)\right| \leq C e^{-\left(c_{0}+c_{1}\right) t} \\
\left|2 \alpha_{+} \alpha_{-} B\left(\mathcal{Y}_{+}, \mathcal{Y}_{-}\right)+Q(g)\right| \leq C e^{-\left(c_{0}+c_{1}\right) t} .
\end{gathered}
$$

By (5.50) and (5.51)

$$
|Q(g)| \leq \begin{cases}C\left(e^{-\left(c_{0}+c_{1}\right) t}+e^{-2 c_{1} t}\right) \leq C e^{-\left(c_{0}+c_{1}\right) t} & \text { if } c_{0}>e_{0} \\ C\left(e^{-\left(c_{0}+c_{1}\right) t}+e^{-\left(e_{0}+c_{1}\right) t}+e^{-2 c_{1} t}\right) \leq C e^{-\left(c_{0}+c_{1}\right) t} & \text { if } c_{0} \leq e_{0}\end{cases}
$$

As a consequence of the coercivity of $Q$ on $G_{\perp}$ (Lemma 5.2), we get the estimate on $\|g\|_{\dot{H}^{1}}$ in (5.52). It remains to show the bounds on $\beta$ and $\gamma$.

Consider the function $\widetilde{\varepsilon}$ defined in (5.49). By assumption (5.38)

$$
\int_{t}^{t+1}\left|(i W, \widetilde{\varepsilon}(s))_{\dot{H}^{1}}\right| d s \leq C e^{-c_{1} t}+\int_{t}^{t+1}\left|(W, \mathcal{L} g(s))_{\dot{H}^{1}}\right| d s .
$$

We have $(W, \mathcal{L} g)_{\dot{H}^{1}}=\operatorname{Re} \int-\Delta(W) \overline{\mathcal{L} g}=-\operatorname{Re} \int \mathcal{L}^{*}(\Delta W) \bar{g}$, where $\mathcal{L}^{*}$ is the $L^{2}$-adjoint of $\mathcal{L}$. Note that $\mathcal{L}^{*}(\Delta W)=\mathcal{L}^{*} W^{p_{c}}$ is in $L^{\frac{2 N}{N+2}}$ (indeed by explicit computation, it is a $C^{\infty}$ function of order $\frac{1}{|x|^{2 N+4}}$ at infinity). Thus, by the estimate on $\|g\|_{\dot{H}^{1}}$ in (5.52),

$$
\left|(\mathcal{L} g(t), W)_{\dot{H}^{1}}\right| \leq C\|g(t)\|_{L^{2^{*}}} \leq C e^{-\left(\frac{c_{0}+c_{1}}{2}\right) t} .
$$

In view of Claim 5.8 and (5.54), we get the bound on $\beta$ in (5.52). An analoguous proof yields the bound on $\gamma$.

Step 4: conclusion. Summing up estimates (5.50), (5.51) and (5.52), we get, in view of decomposition (5.43) of $h$.

$$
\|h(t)\|_{\dot{H}^{1}} \leq \begin{cases}C e^{-\left(\frac{c_{0}+c_{1}}{2}\right) t} & \text { if } c_{0}>e_{0} \\ C\left[e^{-e_{0} t}+e^{-\left(\frac{c_{0}+c_{1}}{2}\right) t}\right] & \text { if } c_{0} \leq e_{0}\end{cases}
$$

Proof of (5.40). Iterating the argument we obtain the bound $\|h(t)\|_{\dot{H}^{1}} \leq C_{\eta} e^{-\left(c_{1}-\eta\right) t}$ if $c_{0}<$ $c_{1}<e_{0}$ or $e_{0}<c_{0}<c_{1}$, which yields (together with Claim 5.10), the desired estimate (5.40) Proof of (5.41). Let us assume $c_{0} \leq e_{0}<c_{1}$. Then the equation on $\alpha_{+}$in (5.47) shows that $e^{e_{0} t} \alpha_{+}(t)$ has a limit $A_{+}$when $t \rightarrow+\infty$. Integrating the equation between $t$ and $+\infty$, we get (in view of Claim 5.11)

$$
A_{+}-e^{e_{0} t} \alpha_{+}(t)=e^{e_{0} t} \int_{t}^{+\infty} \frac{B\left(\mathcal{Y}_{+}, \varepsilon(s)\right)}{B\left(\mathcal{Y}_{+}, \mathcal{Y}_{-}\right)} d s=O\left(e^{\left(e_{0}-c_{1}\right) t}\right) .
$$

By decomposition (5.43) and estimates (5.50) and (5.52), we get $\left\|h(t)-A_{+} e^{-e_{0} t} \mathcal{Y}_{+}\right\|_{\dot{H}^{1}} \leq$ $C e^{-\left(\frac{c_{0}+c_{1}}{2}\right) t}$. Furthermore, $h_{1}(t):=h(t)-A_{+} e^{-e_{0} t} \mathcal{Y}_{+}$satisfies, as $h$, equation (5.37). Thus the estimate (5.40) shown in the preceding step implies (5.41). The proof of Proposition [5.9] is complete. 


\section{Proof of MAin Results}

We now turn to the proof of Theorems 1 and 2, In Subsection 6.2, we show the existence of the solutions $W^{ \pm}$of Theorem 1 by a fixed point, approaching them by approximates solutions $W_{k}^{a}$ of (1.1) constructed in Subsection 6.1 and converging exponentially to $W$ for large $t$. Subsection 6.3 is devoted to the conclusion of the proofs of the theorems.

\subsection{A family of approximate solutions converging to $W$.}

Lemma 6.1. Let $a \in \mathbb{R}$. There exist functions $\left(\Phi_{j}^{a}\right)_{j \geq 1}$ in $\mathcal{S}\left(\mathbb{R}^{N}\right)$, such that $\Phi_{1}^{a}=a \mathcal{Y}_{+}$and if

$$
W_{k}^{a}(t, x):=W(x)+\sum_{j=1}^{k} e^{-j e_{0} t} \Phi_{j}^{a}(x),
$$

then as $t \rightarrow+\infty$,

$$
i \partial_{t} W_{k}^{a}+\Delta W_{k}^{a}+\left|W_{k}^{a}\right|^{p_{c}-1} W_{k}^{a}=O\left(e^{-(k+1) e_{0} t}\right) \text { in } \mathcal{S}\left(\mathbb{R}^{N}\right) .
$$

Remark 6.2. Let $\tilde{\varepsilon}_{k}^{a}:=i \partial_{t} W_{k}^{a}+\Delta W_{k}^{a}+\left|W_{k}^{a}\right|^{p_{c}-1} W_{k}^{a}$. By (6.2) we mean that for all $J, M$, there exists $C_{J, M}>0$ such that $(1+|x|)^{M}\left|\partial_{x}^{J} \tilde{\varepsilon}_{k}^{a}(t, x)\right| \leq C_{J, M} e^{-(k+1) e_{0} t}$.

Proof of the lemma. Let us fix $a \in \mathbb{R}$. To simplify notations, we will omit most of the superscripts $a$. We will construct the functions $\Phi_{j}=\Phi_{j}^{a}$ by induction on $j$. Assume that $\Phi_{1}, \ldots, \Phi_{k}$ are known, and let $v_{k}:=W_{k}-W=\sum_{j=1}^{k} e^{-j e_{0} t} \Phi_{j}(x)$. Assertion (6.2) writes

$$
\varepsilon_{k}:=\partial_{t} v_{k}+\mathcal{L}\left(v_{k}\right)+R\left(v_{k}\right)=O\left(e^{-(k+1) e_{0} t}\right) \text { in } \mathcal{S}\left(\mathbb{R}^{N}\right)
$$

Step 1: $k=1$. Let $\Phi_{1}:=a \mathcal{Y}_{+}$, which is in $\mathcal{S}$ (see Remark 7.2) and $v_{1}(t, x):=e^{-e_{0} t} \Phi_{1}(x)$. We have $\partial_{t} v_{1}+\mathcal{L} v_{1}=0$ and thus

$$
\partial_{t} v_{1}+\mathcal{L} v_{1}+R\left(v_{1}\right)=R\left(v_{1}\right) .
$$

Note that $R\left(v_{1}\right)=W^{p_{c}} J\left(W^{-1} v_{1}\right)$, where $J(z):=-i\left[|1+z|^{p_{c}-1}(1+z)-1-\frac{p_{c}+1}{2} z-\frac{p_{c}-1}{2} \bar{z}\right]$ is real-analytic for $\{|z|<1\}$ and satisfies $J(0)=\partial_{z} J(0)=\partial_{\bar{z}} J(0)=0$. Write

$$
J(z):=\sum_{j_{1}+j_{2} \geq 2} a_{j_{1} j_{2}} z^{j_{1}} \bar{z}^{j_{2}}
$$

with normal convergence of the series and all its derivatives, say for $|z| \leq \frac{1}{2}$. Chose $t_{0}$ such that $\forall t \geq t_{0},\left|v_{1}(t)\right| \leq \frac{1}{2} W$. Then

$$
\forall t \geq t_{0}, \forall x \in \mathbb{R}^{N}, \quad R\left(v_{1}\right)=\sum_{j_{1}+j_{2} \geq 2} a_{j_{1} j_{2}} W^{p_{c}}\left(W^{-1} v_{1}\right)^{j_{1}}\left(W^{-1} \bar{v}_{1}\right)^{j_{2}} .
$$

As a consequence, there exists a constant $C>0$ such that for large $t,\left|R\left(v_{1}\right)\right| \leq C\left|W^{-1} v_{1}\right|^{2}$. Using analoguous inequalities on the derivatives of $R\left(v_{1}\right)$, and the fact that $v_{1}=a e^{-e_{0} t} \Phi_{1}$ with $\Phi_{1} \in \mathcal{S}\left(\mathbb{R}^{N}\right)$, we get $R\left(v_{1}\right)=O\left(e^{-2 e_{0} t}\right)$ in $\mathcal{S}\left(\mathbb{R}^{N}\right)$, which gives (6.2) for $k=1$.

Step 2: induction. Let us assume that $\Phi_{1}, \ldots, \Phi_{k}$ are known and satisfy (6.2) for some $k \geq 1$. To construct $\Phi_{k+1}$, we will first show that there exists $\Psi_{k} \in \mathcal{S}\left(\mathbb{R}^{N}\right)$ (depending only on $\Phi_{1}, \ldots$, $\left.\Phi_{k}\right)$ such that for large $t$

$$
\varepsilon_{k}(x, t)=e^{-(k+1) e_{0} t} \Psi_{k}(x)+O\left(e^{-(k+2) e_{0} t}\right) \text { in } \mathcal{S}\left(\mathbb{R}^{N}\right) .
$$


Indeed by (6.2)

$$
\varepsilon_{k}(t, x)=\sum_{j=1}^{k} e^{-j e_{0} t}\left(-j e_{0} \Phi_{j}(x)+\mathcal{L} \Phi_{j}(x)\right)+R\left(v_{k}(t, x)\right) .
$$

All the functions $\Phi_{j}$ are in $\mathcal{S}\left(\mathbb{R}^{N}\right)$, so that for large $t$, and all $x,\left|v_{k}(t, x)\right| \leq \frac{1}{2} W(x)$. Furthermore $R\left(v_{k}\right)=W^{p_{c}} J\left(W^{-1} v_{k}\right)$, and by the development (6.3) of $J$ we get by (6.6) that there exist functions $F_{j} \in \mathcal{S}\left(\mathbb{R}^{N}\right)$ such that for large $t$

$$
\varepsilon_{k}(t, x)=\sum_{j=1}^{k+1} e^{-j e_{0} t} F_{j}(x)+O\left(e^{-(k+2) t}\right) \text { in } \mathcal{S}\left(\mathbb{R}^{N}\right) .
$$

By (6.21) at rank $k, F_{j}=0$ for $j \leq k$ which shows (6.5) with $\Phi_{k}=F_{k+1}$.

By Corollary 5.3, $(k+1) e_{0}$ is not in the spectrum of $\mathcal{L}$. Let

$$
\Phi_{k+1}:=-\left(\mathcal{L}-(k+1) e_{0}\right)^{-1} \Psi_{k}
$$

which belongs to $\mathcal{S}\left(\mathbb{R}^{N}\right)$ (see Remark 7.2) and is uniquely determined by $\Phi_{1}, \ldots, \Phi_{k}$. By definition, $v_{k+1}=v_{k}+e^{-(k+1) e_{0} t} \Phi_{k+1}$. Furthermore,

$$
\begin{aligned}
\varepsilon_{k+1} & :=\partial_{t} v_{k+1}+\mathcal{L} v_{k+1}+R\left(v_{k+1}\right) \\
& =\partial_{t} v_{k}+\mathcal{L} v_{k}+R\left(v_{k}\right)-(k+1) e_{0} \Phi_{k+1} e^{-(k+1) e_{0} t}+\mathcal{L} \Phi_{k+1} e^{-(k+1) e_{0} t}+R\left(v_{k+1}\right)-R\left(v_{k}\right) \\
& =\varepsilon_{k}-e^{-(k+1) e_{0} t} \Psi_{k}+R\left(v_{k+1}\right)-R\left(v_{k}\right) .
\end{aligned}
$$

By (6.5),$\varepsilon_{k}-e^{-(k+1) e_{0} t} \Psi_{k}=O\left(e^{-(k+2) e_{0} t}\right)$ in $\mathcal{S}\left(\mathbb{R}^{N}\right)$. Writing as before $R=W^{p_{c}} J(W \cdot)$, and using the developpment (6.3) of $J$, we get that $R\left(v_{k+1}\right)-R\left(v_{k}\right)=O\left(e^{-(k+2) e_{0} t}\right)$ in $\mathcal{S}\left(\mathbb{R}^{N}\right)$ which yields (6.2) at rank $k+1$. The proof is complete.

\subsection{Contraction argument near an approximate solution.}

Proposition 6.3. Let $a \in \mathbb{R}$. There exists $k_{0}>0$ such that for any $k \geq k_{0}$, there exists $t_{k} \geq 0$ and a solution $W^{a}$ of (1.1) such that for $t \geq t_{k}$,

$$
\left\|\nabla\left(W^{a}-W_{k}^{a}\right)\right\|_{Z(t,+\infty)} \leq e^{-\left(k+\frac{1}{2}\right) e_{0} t} .
$$

Furthermore, $W^{a}$ is the unique solution of (1.1) satisfying (6.7) for large $t$. Finally, $W^{a}$ is independent of $k$ and satisfies for large $t$,

$$
\left\|W^{a}(t)-W-a e^{-e_{0} t} \mathcal{Y}_{+}\right\|_{\dot{H}^{1}} \leq e^{-\frac{3}{2} e_{0} t} .
$$

Proof. Step 1: transformation into a fixed-point problem. As in the preceding proof, we will fix $a \in \mathbb{R}$ and omit most of the superscripts $a$. Let

$$
h:=W^{a}-W_{k}^{a} .
$$

The function $W^{a}$ is solution of (1.1) if and only if $w^{a}:=W^{a}-W$ is solution of (5.2). Substracting equations (5.2) on $w^{a}$ and (6.21) on $v_{k}:=W_{k}^{a}-W$, we get that $W^{a}$ satisfies (1.1) if and only if $h=w^{a}-v_{k}$ satisfies $\partial_{t} h+\mathcal{L} h=-R\left(v_{k}+h\right)+R\left(v_{k}\right)+\varepsilon_{k}$ (see (6.2]) for the definition of $\varepsilon_{k}$ ). This may be rewritten (recalling (5.19) for the definition of $\mathcal{V}$ )

$$
i \partial_{t} h+\Delta h=-\mathcal{V}(h)-i R\left(v_{k}+h\right)+i R\left(v_{k}\right)+i \varepsilon_{k} .
$$


Thus the existence of a solution $W^{a}$ of (1.1) satisfying (6.7) for $t \geq t_{k}$ may be written as the following fixed-point problem

$$
\begin{aligned}
& \forall t \geq t_{k}, \quad h(t)=\mathcal{M}_{k}(h)(t) \text { and }\|\nabla h\|_{Z(t,+\infty)} \leq e^{-\left(k+\frac{1}{2}\right) e_{0} t} \\
& \text { where } \mathcal{M}_{k}(h)(t):=-\int_{t}^{+\infty} e^{i(t-s) \Delta}\left[i \mathcal{V}(h(s))-R\left(v_{k}(s)+h(s)\right)+R\left(v_{k}(s)\right)-\varepsilon_{k}(s)\right] d s
\end{aligned}
$$

Let us fix $k$ and $t_{k}$. Consider

$$
\begin{aligned}
& E_{Z}^{k}:=\left\{h \in S\left(t_{k},+\infty\right), \nabla h \in Z\left(t_{k},+\infty\right) ;\|h\|_{E_{Z}^{k}}:=\sup _{t \geq t_{k}} e^{\left(k+\frac{1}{2}\right) e_{0} t}\|\nabla h\|_{Z(t,+\infty)}<\infty\right\} \\
& B_{Z}^{k}:=\left\{h \in E_{Z}^{k},\|h\|_{E_{Z}^{k}} \leq 1\right\} .
\end{aligned}
$$

The space $E_{Z}^{k}$ is clearly a Banach space. In view of (6.9), it is sufficient to show that if $t_{k}$ and $k$ are large enough, the mapping $\mathcal{M}_{k}$ is a contraction on $B_{Z}^{k}$. This is the aim of the next step.

Step 2: contraction property. Note that by Strichartz inequality (5.22), there is a constant $C^{*}>0$ such that if $g, h \in E_{Z}^{k}, k \geq 1$,

$$
\begin{aligned}
\left\|\nabla\left(\mathcal{M}_{k}(h)\right)\right\|_{Z(t,+\infty) \leq C^{*}} & {\left[\|\nabla(\mathcal{V}(h))\|_{N(t,+\infty)}\right.} \\
& \left.+\left\|\nabla\left(R\left(v_{k}+h\right)-R\left(v_{k}\right)\right)\right\|_{N(t,+\infty)}+\left\|\nabla \varepsilon_{k}\right\|_{N(t,+\infty)}\right] \\
\left\|\nabla\left(\mathcal{M}_{k}(g)-\mathcal{M}_{k}(h)\right)\right\|_{Z(t,+\infty) \leq C^{*}} & {\left[\|\nabla(\mathcal{V}(g-h))\|_{N(t,+\infty)}\right.} \\
+ & \left.\left\|\nabla\left(R\left(v_{k}+h\right)-R\left(v_{k}+g\right)\right)\right\|_{N(t,+\infty)}\right] .
\end{aligned}
$$

Claim 6.4. There exists $k_{0}>0$ such that for $k \geq k_{0}$ the following holds: for all $h \in E_{Z}^{k}$

$$
\|\nabla(\mathcal{V}(h))\|_{N(t,+\infty)} \leq \frac{1}{4 C^{*}} e^{-\left(k+\frac{1}{2}\right) e_{0} t}\|h\|_{E_{Z}^{k}} ;
$$

and there exists a constant $C_{k}$, depending only on $k$ such that for all $f, g \in B_{Z}^{k}$ and $t \geq t_{k}$

$$
\begin{gathered}
\left\|\nabla\left(R\left(v_{k}+g\right)-R\left(v_{k}+h\right)\right)\right\|_{N(t,+\infty)} \leq C_{k} e^{-\left(k+\frac{3}{2}\right) e_{0} t}\|g-h\|_{E_{Z}^{k}}, \\
\left\|\nabla \varepsilon_{k}\right\|_{N(t,+\infty)} \leq C_{k} e^{-(k+1) e_{0} t} .
\end{gathered}
$$

Let us first assume Claim 6.4 and prove the proposition. Chose $k \geq k_{0}$. By (6.10), (6.11), (6.12), (6.13) and (6.14), we get, if $g, h \in B_{Z}^{k}$

$$
\begin{gathered}
\left\|\mathcal{M}_{k}(h)\right\|_{E_{Z}^{k}} \leq\left(\frac{1}{4}+C^{*} C_{k} e^{-e_{0} t_{k}}+C^{*} C_{k} e^{-\frac{1}{2} e_{0} t_{k}}\right), \\
\left\|\mathcal{M}_{k}(g)-\mathcal{M}_{k}(h)\right\|_{E_{Z}^{k}} \leq\|g-h\|_{E_{Z}^{k}}\left(\frac{1}{2}+C^{*} C_{k} e^{-e_{0} t_{k}}\right),
\end{gathered}
$$

which shows, chosing a larger $t_{k}$ if necessary, that $\mathcal{M}_{k}$ is a contraction of $B_{Z}^{k}$.

Thus, for each $k \geq k_{0}$, (1.1) has an unique solution $W^{a}$ satisfying (6.7) for $t \geq t_{k}$. The preceding proof clearly remains valid taking a larger $t_{k}$, so that the uniqueness still holds in the class of solutions of (1.1) satisfying (6.7) for $t \geq t_{k}^{\prime}$, where $t_{k}^{\prime}$ is any real number larger than $t_{k}$. Let $k<\tilde{k}$ and $W^{a}, \widetilde{W}^{a}$ be the solutions of (1.1) constructed above for $k$ and $\tilde{k}$ respectively. Then, $\widetilde{W}^{a}$ although satisfies (6.7) for large $t$, so that the uniqueness in the fixed-point shows 
that $W^{a}(t)=\widetilde{W}^{a}(t)$, for large $t$ and thus, by uniqueness in (1.1), that $W^{a}=\widetilde{W}^{a}$. This shows that $W^{a}$ does not depend on $k$.

It remains to show (6.8). Let $k>0$ be a large integer and $h \in B_{Z}^{k}$. By Strichartz inequality (5.23), and the definition of $\mathcal{M}_{k}$, we have, for $t \geq t_{k}$,

$$
\left\|\mathcal{M}_{k}(h)(t)\right\|_{\dot{H}^{1}} \leq\left\|\nabla\left(i \mathcal{V}(h)-R\left(v_{k}+h\right)+R\left(v_{k}\right)-\varepsilon_{k}\right)\right\|_{N(t,+\infty)} .
$$

As a consequence of Claim 6.4 and the fact that $\|h\|_{E_{Z}}^{k} \leq 1$, we get

$$
\left\|\mathcal{M}_{k}(h)(t)\right\|_{\dot{H}^{1}} \leq C\left(e^{-\left(k+\frac{1}{2}\right) e_{0} t}\|h\|_{E_{Z}^{k}}+e^{-(k+1) e_{0} t}\right) \leq C e^{-\left(k+\frac{1}{2}\right) e_{0} t} .
$$

Using the preceding inequality on $h=W^{a}-W_{k}^{a}$ (which satisfies $h=\mathcal{M}_{k}(h)$ ), and noting that $W_{k}^{a}=W+a e^{-e_{0} t} \mathcal{Y}_{+}+O\left(e^{-2 e_{0} t}\right)$ in $\dot{H}^{1}$, we get directly (6.8). To complete the proof of the proposition, it remains to show Claim 6.4,

Proof of Claim 6.4. Estimate (6.14) follows immediately from (6.21).

Let us show (6.13). By Lemma 5.6.

$$
\begin{aligned}
\left\|\nabla\left(R\left(v_{k}+g\right)-R\left(v_{k}+h\right)\right)\right\|_{N(t, t+1)} \leq(A)\|\nabla(g-h)\|_{Z(t, t+1)}, & \\
(A):=C\left(\|\nabla g\|_{Z(t, t+1)}\right. & +\|\nabla h\|_{Z(t, t+1)}+\left\|\nabla v_{k}\right\|_{Z(t, t+1)} \\
& \left.+\|\nabla g\|_{Z(t, t+1)}^{p_{c}-1}+\|\nabla h\|_{Z(t, t+1)}^{p_{c}-1}+\left\|\nabla v_{k}\right\|_{Z(t, t+1)}^{p_{c}-1}\right) .
\end{aligned}
$$

By the explicit form of $v_{k}$ and the fact that $g, h \in B_{Z}^{k}$, we get

$$
(A) \leq C_{k}^{\prime} e^{-e_{0} t}
$$

where $C_{k}^{\prime}$ only depends on $k$. Combining (6.15) and (6.16), we get

$$
\left\|\nabla\left(R\left(v_{k}+g\right)-R\left(v_{k}+h\right)\right)\right\|_{N(t, t+1)} \leq C_{k}^{\prime} e^{-e_{0} t}\|\nabla(g-h)\|_{Z(t, t+1)} \leq C_{k}^{\prime \prime} e^{-\left(k+\frac{3}{2}\right) e_{0} t}\|g-h\|_{E_{Z}}
$$

which gives (6.13) in view of Claim 5.8 .

It remains to show (6.12). Let $\tau_{0}>0$. By Lemma [5.5, there exists a constant $C_{2}>0$ such that

$$
\|\nabla(\mathcal{V} h)\|_{N\left(t, t+\tau_{0}\right)} \leq C_{2} \tau_{0}^{\frac{N}{N+2}}\|\nabla h\|_{Z\left(t, t+\tau_{0}\right)} \leq C_{2} \tau_{0}^{\frac{N}{N+2}} e^{-\left(k+\frac{1}{2}\right) e_{0} t}\|h\|_{E_{Z}^{k}}
$$

By Claim 5.8,

$$
\|\nabla(\mathcal{V} h)\|_{N(t,+\infty)} \leq \frac{C_{2} e^{-\left(k+\frac{1}{2}\right) e_{0} t}}{1-e^{-\left(k+\frac{1}{2}\right) e_{0} \tau_{0}}} \tau_{0}^{\frac{N}{N+2}}\|h\|_{E_{Z}^{k}} .
$$

Chosing $\tau_{0}$ and $k_{0}$ such that $C_{2} \tau_{0}^{\frac{N}{N+2}}=\frac{1}{8 C_{1}}$ and $e^{-\left(k_{0}+\frac{1}{2}\right) e_{0} \tau_{0}} \leq \frac{1}{2}$, we get (6.12) for $k \geq k_{0}$.

\subsection{Conclusion of the proofs of the theorems.}

Proof of Theorem 1. Denote as before $\mathcal{Y}_{1}:=\operatorname{Re} \mathcal{Y}_{+}=\operatorname{Re} \mathcal{Y}_{-}$. Note that $\left(W, \mathcal{Y}_{1}\right)_{\dot{H}^{1}} \neq 0$. Indeed, if $\left(W, \mathcal{Y}_{1}\right)_{\dot{H}^{1}}=0$, then by the equation $\Delta W=-W^{p_{c}}$, we would have $B\left(W, \mathcal{Y}_{+}\right)=B\left(W, \mathcal{Y}_{-}\right)=0$ so that $W \in G_{\perp}$, which contradicts, in view of Lemma 5.2, the fact that $Q(W)=-\frac{2}{(N-2) C_{N}^{N}}<0$. Replacing $\mathcal{Y}_{ \pm}$by $-\mathcal{Y}_{ \pm}$if necessary, we may assume

$$
\left(W, \mathcal{Y}_{1}\right)_{\dot{H}^{1}}>0 \text {. }
$$


Let

$$
W^{ \pm}:=W^{ \pm 1}
$$

which yields two solutions of (1.1) for large $t>0$. Then all the conditions of Theorem 1 are satisfied. Indeed (1.8) follows from the conservation of the energy and the fact that $W^{a}$ tends to $W$ in $\dot{H}^{1},(1.9)$ is an immediate consequence (6.8). Furthermore, again by (6.8),

$$
\left\|W^{a}\right\|_{\dot{H}^{1}}^{2}=\|W\|_{\dot{H}^{1}}^{2}+2 a e^{-e_{0} t}\left(W, \mathcal{Y}_{1}\right)_{\dot{H}^{1}}+O\left(e^{-\frac{3}{2} e_{0} t}\right),
$$

which shows, together with (6.17), that for large $t>0$,

$$
\left\|W^{+}(t)\right\|_{\dot{H}^{1}}>0, \quad\left\|W^{-}(t)\right\|_{\dot{H}^{1}}<0 .
$$

From Remark 1.2, these inequalities remain valids for every $t$ in the intervals of existence of $W^{+}$ and $W^{-}$. Finally $T_{-}\left(W^{-}\right)=-\infty$ by Proposition 2.1 and $\|u\|_{S(-\infty, 0)}<\infty$ by Corollary 3.2 .

It remains to show that in the case $N=5, T_{-}\left(W^{+}\right)<\infty$. For this we will show that for any $a$ and any $t$ in the interval of definition of $W^{a}$,

$$
N=5 \Longrightarrow W^{a}(t) \in L^{2}\left(\mathbb{R}^{5}\right)
$$

Consider as in Subsection 2.3 a positive radial function $\psi$ on $\mathbb{R}^{5}$, such that $\psi=1$ if $|x| \leq 1$ and $\psi=0$ if $|x| \geq 2$. Define, for $R>0$ and large $t$,

$$
F_{R}(t):=\int_{\mathbb{R}^{5}}\left|W^{a}(t, x)\right|^{2} \psi\left(\frac{x}{R}\right) d x .
$$

Then, $W^{a}$ being a solution of (1.1),

$$
\begin{aligned}
F_{R}^{\prime}(t) & =\frac{2}{R} \operatorname{Im} \int W^{a} \nabla \bar{W}^{a} \cdot(\nabla \psi)\left(\frac{x}{R}\right) d x=\frac{2}{R} \operatorname{Im} \int W \nabla\left(\bar{W}^{a}-W\right) \cdot(\nabla \psi)\left(\frac{x}{R}\right) d x \\
& +\frac{2}{R} \operatorname{Im} \int\left(W^{a}-W\right) \nabla W \cdot(\nabla \psi)\left(\frac{x}{R}\right) d x+\frac{2}{R} \operatorname{Im} \int\left(W^{a}-W\right) \nabla\left(\bar{W}^{a}-\bar{W}\right) \cdot(\nabla \psi)\left(\frac{x}{R}\right) d x .
\end{aligned}
$$

Using that by (6.8), $\left\|W^{a}(t)-W\right\|_{\dot{H}^{1}} \leq C e^{-e_{0} t}$, we get, by Hardy inequality

$$
\left|F_{R}^{\prime}(t)\right| \leq C\left\|W^{a}(t)-W\right\|_{\dot{H}^{1}}\left(\left\|W^{a}(t)\right\|_{\dot{H}^{1}}+\|W\|_{\dot{H}^{1}}\right) \leq C e^{-e_{0} t},
$$

with a constant $C$ independent of $R$, and thus, integrating between a large $t$ and $+\infty$,

$$
\left.\left|F_{R}(t)-\int_{\mathbb{R}^{N}}\right| W(x)\right|^{2} \psi\left(\frac{x}{R}\right) d x \mid \leq C e^{-e_{0} t} .
$$

Letting $R$ goes to $+\infty$, we get (6.18) and $\left\|W^{a}(t)\right\|_{L^{2}}=\|W\|_{L^{2}}$. In particular $W^{+}(t) \in L^{2}\left(\mathbb{R}^{5}\right)$, and thus, by Corollary 4.2, $T_{-}\left(W^{+}\right)<\infty$ which concludes the proof of Theorem 1 ,

Proof of Theorem 2, Let us first prove:

Lemma 6.5. If $u$ is a solution of (1.1) satisfying

$$
\|u(t)-W\|_{\dot{H}^{1}} \leq C e^{-\gamma_{0} t}, \quad E(u)=E(W)
$$

then

$$
\exists ! a \in \mathbb{R}, \quad u=W^{a} .
$$

Corollary 6.6. For any $a \neq 0$, there exists $T_{a} \in \mathbb{R}$ such that

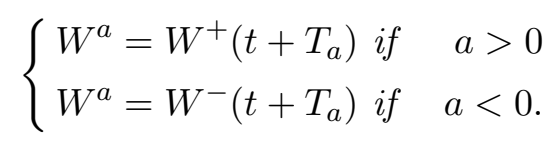


Proof. Let $u=W+v$ be a solution of (1.1) for $t \geq t_{0}$ satisfying (6.19). Recall that $v$ satisfies equation (5.2).

Step 1. We show that there exists $a \in \mathbb{R}$ such that

$$
\forall \eta>0, \quad\left\|v(T)-a e^{-e_{0} T} \mathcal{Y}_{+}\right\|_{\dot{H}^{1}}+\left\|\nabla\left(v(t)-a e^{-e_{0} t} \mathcal{Y}_{+}\right)\right\|_{Z(T,+\infty)} \leq C_{\eta} e^{-(2-\eta) e_{0} T} .
$$

Indeed we will show

$$
\|v(t)\|_{\dot{H}^{1}} \leq C e^{-e_{0} t}, \quad\|R(v(t))\|_{L^{\frac{2 N}{N+2}}}+\|\nabla(R(v))\|_{N(t,+\infty)} \leq C e^{-2 e_{0} t} .
$$

Assuming (6.22), we are in the setting of Proposition 5.9 with $h=v, \varepsilon=-R(v), c_{0}=e_{0}$ and $c_{1}=2 e_{0}$. The conclusion (5.41) of the proposition would then yield (6.21). It remains to prove (6.22).

By Lemma 5.6, Claim 5.10 and Claim 5.8, the bound on $R(v)$ in (6.22) follows from the bound on $\|v(t)\|_{\dot{H}^{1}}$, so that we only need to show this first bound.

By Lemma 5.7, assumption (6.19) implies $\|v(t)\|_{\dot{H}^{1}}+\|\nabla v\|_{Z(t,+\infty)} \leq C e^{-\gamma_{0} t}$. By Lemma 5.6 and Claim 5.8

$$
\|R(v(t))\|_{L^{\frac{2 N}{N+2}}}+\|\nabla(R(v))\|_{N(t,+\infty)} \leq C e^{-2 \gamma_{0} t} .
$$

Thus we can apply Proposition 5.9, showing that

$$
\|v(t)\|_{\dot{H}^{1}} \leq C\left(e^{-e_{0} t}+e^{-\frac{3}{2} \gamma_{0} t}\right) .
$$

If $\frac{3}{2} \gamma_{0} \geq e_{0}$ the proof of (6.22) is complete. If not, assumption (6.19) on $v$ holds with $\frac{3}{2} \gamma_{0}$ instead of $\gamma_{0}$, and an iteration argument yields the result. The proof of (6.22) is complete, which concludes Step 1.

Step 2. Let us show

$$
\forall m>0, \exists t_{0}>0, \forall t \geq t_{0}, \quad\left\|u(t)-W^{a}(t)\right\|_{\dot{H}^{1}}+\left\|\nabla\left(u-W^{a}\right)\right\|_{Z(t,+\infty)} \leq e^{-m t} .
$$

This will show that $u=W^{a}$, by uniqueness in Proposition 6.3. According to Step 1, (6.23) holds for $m=\frac{3}{2} e_{0}$. Let us assume (6.23) holds for some $m=m_{1}>e_{0}$. We will show that it holds for $m=m_{1}+\frac{e_{0}}{2}$, which will yield (6.23) by iteration and conclude the proof.

Write $v(t):=u(t)-W, w^{a}(t):=W^{a}(t)-W\left(\right.$ so that in particular $\left.u-W^{a}=v-w^{a}\right)$. Then

$$
\partial_{t}\left(v-w^{a}\right)+\mathcal{L}\left(v-w^{a}\right)=-R(v)+R\left(w^{a}\right) .
$$

We have assumed $\left\|v(t)-w^{a}(t)\right\|_{\dot{H}^{1}}+\left\|\nabla\left(v-w^{a}\right)\right\|_{Z(t,+\infty)} \leq e^{-m_{1} t}$. According to Lemma 5.6 and Claim 5.8

$$
\left\|\nabla\left(R(v)-R\left(w^{a}\right)\right)\right\|_{N(t,+\infty)}+\left\|R(v(t))-R\left(w^{a}(t)\right)\right\|_{L^{\frac{2 N}{N+2}}} \leq C e^{-\left(m_{1}+e_{0}\right) t} .
$$

Then by Proposition 5.9

$$
\left\|v(t)-w^{a}(t)\right\|_{\dot{H}^{1}}+\left\|\nabla\left(v-w^{a}\right)\right\|_{Z(t,+\infty)} \leq C e^{-\left(m_{1}+\frac{3}{4} e_{0}\right) t}
$$

which yields (6.23) with $m=m_{1}+\frac{e_{0}}{2}$. By iteration, (6.23) holds for any $m>0$. Using this with $m=\left(k_{0}+1\right) e_{0}$ (where $k_{0}$ is given by Proposition 6.3), we get that for large $t>0$

$$
\left\|\nabla\left(u-W_{k_{0}}^{a}\right)\right\|_{Z(t,+\infty)} \leq e^{-\left(k_{0}+\frac{1}{2}\right) e_{0} t} .
$$

By uniqueness in Proposition 6.3, we get as announced that $u=W^{a}$ which concludes the proof of the lemma. 
Proof of Corollary [6.6. Let $a \neq 0$ and chose $T_{a}$ such that $|a| e^{-e_{0} T_{a}}=1$. By (6.8),

$$
\left\|W^{a}\left(t+T_{a}\right)-W \mp e^{-e_{0} t} \mathcal{Y}_{+}\right\|_{\dot{H}^{1}} \leq C e^{-\frac{3}{2} e_{0} t} .
$$

Furthermore, $W^{a}\left(\cdot+T_{a}\right)$ satisfies the assumptions of Lemma 6.5, which shows that there exists $a^{\prime}$ such that $W^{a}\left(\cdot+T_{a}\right)=W^{a^{\prime}}$. By (6.24), $a^{\prime}=1$ if $a>0$ and $a^{\prime}=-1$ if $a<0$, hence (6.20).

Let us turn to the proof of Theorem 2, Point (b) is an immediate consequence of the variational characterization of $W$ ([Aub76], Tal76]).

Let us show (a). Let $u$ be a solution of (1.1) such that $E\left(u_{0}\right)=E(W)$ and $\left\|u_{0}\right\|_{\dot{H}^{1}}<$ $\|W\|_{\dot{H}^{1}}$. Assume that $\|u\|_{S(\mathbb{R})}=\infty$. Replacing if necessary $u(t)$ by $\bar{u}(-t)$, we may assume that $\|u\|_{S(0,+\infty)}=\infty$. Then by Proposition [3.1, there exist $\theta_{0} \in \mathbb{R}, \mu_{0}>0$, and $c, C>0$ such that $\left\|u(t)-W_{\left[\theta_{0}, \mu_{0}\right]}\right\|_{\dot{H}^{1}} \leq C e^{-c t}$. This shows that $u_{\left[-\theta_{0}, \mu_{0}^{-1}\right]}$ fullfills the assumptions of Lemma 6.5, Using that $\|u\|_{\dot{H}^{1}}<\|W\|_{\dot{H}^{1}}$, this implies that there exists $a<0$ such that $u_{\left[-\theta_{0}, \mu_{0}^{-1}\right]}=W^{a}$. Thus by Corollary 6.6,

which shows (at).

$$
u(t)=W_{\left[\theta_{0}, \mu_{0}\right]}^{-}\left(t+T_{a}\right)
$$

The proof of (ㄷ) is similar. Indeed if $u$ is a solution of (1.1) defined on $[0,+\infty)$ and such that $E\left(u_{0}\right)=E(W),\left\|u_{0}\right\|_{\dot{H}^{1}}>\|\nabla W\|_{\dot{H}^{1}}$ and $u_{0} \in L^{2}$, then by Proposition 4.1, $\left\|u(t)-W_{\left[\theta_{0}, \mu_{0}\right]}\right\|_{\dot{H}^{1}} \leq$ $C e^{-c t}$, which shows using Lemma 6.5 and the same argument as before that for some $t_{0} \in \mathbb{R}$,

$$
u(t)=W_{\left[\theta_{0}, \mu_{0}\right]}^{+}\left(t+t_{0}\right) .
$$

The proof of Theorem 2 is complete.

\section{Appendix}

\subsection{Proofs of some results of decomposition near $W$.}

7.1.1. Proof of Lemma 3.6. Let us first show the lemma when $f$ is close to $W$. Consider the following functionals on $\mathbb{R} \times(0,+\infty) \times \dot{H}^{1}$ :

$$
J_{0}:(\theta, \mu, f) \mapsto\left(f_{[\theta, \mu]}, i W\right)_{\dot{H}^{1}}, \quad J_{1}:(\theta, \mu, f) \mapsto\left(f_{[\theta, \mu]}, W_{1}\right)_{\dot{H}^{1}}
$$

Then, by (3.18)

$$
\begin{array}{ll}
\frac{\partial J_{0}}{\partial \theta}(0,1, W)=\int|\nabla W|^{2} & \frac{\partial J_{0}}{\partial \mu}(0,1, W)=0 \\
\frac{\partial J_{0}}{\partial \mu}(0,1, W)=0 & \frac{\partial J_{1}}{\partial \mu}(0,1, W)=-\int\left|\nabla W_{1}\right|^{2} .
\end{array}
$$

Furthermore, $J_{0}(0,1, W)=J_{1}(0,1, W)=0$. Thus by the Implicit Function Theorem there exists $\varepsilon_{0}, \eta_{0}>0$ such that for $h \in \dot{H}^{1}$ :

$$
\|h-W\|_{\dot{H}^{1}}<\varepsilon_{0} \Longrightarrow \exists !(\theta, \mu), \quad|\theta|+|\mu-1| \leq \eta_{0} \text { and }\left(h_{[\theta, \mu]}, i W\right)_{\dot{H}^{1}}=\left(h_{[\theta, \mu]}, W_{1}\right)_{\dot{H}^{1}}=0 .
$$

Let $f$ be as in the proposition. By the variational characterization of $W$, if $\mathrm{d}(f)$ is small enough, we can choose $\mu_{1}$ and $\theta_{1}$ such that $f_{\left[\theta_{1}, \mu_{1}\right]}=W+g,\|g\|_{\dot{H}^{1}} \leq \varepsilon(\mathrm{d}(f))$, and we are now reduced to the preceding case. The assertions on the uniqueness of $(\theta, \mu)$ and the regularity of the mapping $f \mapsto(\theta, \mu)$ follows from the Implicit Functions Theorem. The proof of Lemma 3.6 is complete. 
7.1.2. Proof of Lemma 3.7. Take $u$ as in Lemma 3.7 and let

$$
v(t):=u_{[\theta(t), \mu(t)]}(t)-W=\tilde{u}(t)+\alpha(t) W .
$$

Proof of (3.22). In this part of the proof, $t$ is just a parameter and we will not write it for the sake of simplicity. By (7.1),

$$
\|v\|_{\dot{H}^{1}}^{2}=\alpha^{2}\|W\|_{\dot{H}^{1}}^{2}+\|\tilde{u}\|_{\dot{H}^{1}}^{2} .
$$

To get a second relation between $\|v\|_{\dot{H}^{1}}, \alpha$ and $\|\tilde{u}\|_{\dot{H}^{1}}$, we use the equation $E(W)=E(W+$ $v)$ together with (3.17). Denote by $\tilde{u}_{1}$ and $\tilde{u}_{2}$ the real and imaginary parts of $\tilde{u}$. By the orthogonality of $\tilde{u}_{1}$ and $\tilde{u}_{2}$ with $W$ in $\dot{H}^{1}$, and the equation $\Delta W+W^{p_{c}}=0$ we have

$$
\int \nabla W \cdot \nabla \tilde{u}_{1}=\int \nabla W \cdot \nabla \tilde{u}_{2}=\int W^{p_{c}} \tilde{u}_{1}=\int W^{p_{c}} \tilde{u}_{2}=0 .
$$

Thus $W$ and $\tilde{u}$ are $Q$-orthogonal and $Q(v)=Q(\tilde{u}+\alpha W)=-|Q(W)| \alpha^{2}+Q(\tilde{u})$. This yields, using (3.17), $\left|\alpha^{2}\right| Q(W)|-Q(\tilde{u})| \leq C\|v\|_{\dot{H}^{1}}^{3}$. By the coercivity of $Q$ on $H^{\perp}$ (Claim 3.5) which implies $Q(\tilde{u}) \approx\|\tilde{u}\|_{\dot{H}^{1}}^{2}$, we get

$$
\|\tilde{u}\|_{\dot{H}^{1}}^{2} \leq C\left(\|v\|_{\dot{H}^{1}}^{3}+\alpha^{2}\right), \quad \alpha^{2} \leq C\left(\|\tilde{u}\|_{\dot{H}^{1}}^{2}+\|v\|_{\dot{H}^{1}}^{3}\right) .
$$

It follows from the variational characterization of $W$ that $\|v\|_{\dot{H}^{1}}$ is small when $\mathrm{d}(u)$ is small. By (7.2) and (7.3), we get, for small $\mathrm{d}(u)$,

$$
|\alpha| \approx\|v\|_{\dot{H}^{1}} \approx\|\tilde{u}\|_{\dot{H}^{1}} .
$$

This is the first part of (3.22). It remains to show the estimate on $\mathrm{d}(u)$. Developing the equation $\|W+v\|_{\dot{H}^{1}}^{2}=\|W\|_{\dot{H}^{1}}^{2}+\mathrm{d}(u)$ we get,

$$
\|v\|_{\dot{H}^{1}}^{2}+2(v, W)_{\dot{H}^{1}}=\|v\|_{\dot{H}^{1}}^{2}+2 \alpha=\mathrm{d}(u)
$$

which gives, thanks to (7.4), the desired result. The proofs of (3.22) is complete. Proof of (3.23). Let us consider the self-similar variables $y$ and $s$ defined by

$$
\mu(t) y=x, \quad d s=\mu^{2}(t) d t .
$$

Then (1.1) may be rewritten

$$
i \partial_{s} u_{[\theta, \mu]}+\Delta_{y} u_{[\theta, \mu]}+\left|u_{[\theta, \mu]}\right|^{p_{c}-1} u_{[\theta, \mu]}+\theta_{s} u_{[\theta, \mu]}+i \frac{\mu_{s}}{\mu}\left(\frac{N-2}{2} u_{[\theta, \mu]}+y \cdot \nabla u_{[\theta, \mu]}\right)=0
$$

where the subscript $s$ denotes the derivative with respect to $s$ and $\Delta_{y}$ the Laplace operator with respect to the new space variable $y$.

We much show

$$
\left|\alpha_{s}(s)\right|+\left|\theta_{s}(s)\right|+\left|\frac{\mu_{s}}{\mu}(s)\right| \leq C|d(u(s))| .
$$

For any complex-valued function $f$, we will write $f_{1}:=\operatorname{Re} f, f_{2}:=\operatorname{Im} f$. Writing $u_{[\theta, \mu]}=W+v$, we get

$$
\partial_{s} v+\mathcal{L} v+R(v)-\theta_{s} i W-\theta_{s} i v+\frac{\mu_{s}}{\mu} W_{1}+\frac{\mu_{s}}{\mu}\left(\frac{N-2}{2} v+y \cdot \nabla v\right)=0 .
$$


Where the linear operator $\mathcal{L}$ and the remainder term $R$ are defined by (5.2). We will need the following bound on $R(v)$ (see Lemma [5.6)

$$
\|R(v)\|_{L^{\frac{2 N}{N+2}}} \leq C\left(\|v\|_{\dot{H}^{1}}^{2}+\|v\|_{\dot{H}^{1}}^{p_{c}}\right) .
$$

Writing $v=\tilde{u}+\alpha(s) W$ and keeping in the left-hand side only the terms that are linear in $\tilde{u}, \alpha$, $\alpha_{s}, \theta, \theta_{s}$ and $\mu_{s} / \mu$, we get

$$
\begin{aligned}
\partial_{s} \tilde{u}_{1}+i \partial_{s} \tilde{u}_{2}+\alpha_{s} W+(\Delta & \left.+W^{p_{c}-1}\right) \tilde{u}_{2}-i\left(\Delta+p_{c} W^{p_{c}-1}\right) \tilde{u}_{1}-i \alpha\left(p_{c}-1\right) W^{p_{c}} \\
& -\theta_{s} i W+\frac{\mu_{s}}{\mu} W_{1}=-R(v)+\theta_{s} i v-\frac{\mu_{s}}{\mu}\left(\frac{N-2}{2} v+y \cdot \nabla v\right) .
\end{aligned}
$$

In view of estimates (3.22), it is easy to see that the $\dot{H}^{1}$-scalar products of the right-hand term by $W, i W$ and $W_{1}$ are bounded up to a constant by $\varepsilon(s)$, where $\varepsilon(s)$ is defined by

$$
\varepsilon(s):=|\mathrm{d}|\left(|\mathrm{d}|+\left|\theta_{s}(s)\right|+\left|\frac{\mu_{s}}{\mu}(s)\right|\right), \quad \mathrm{d}:=\mathrm{d}(u(s)) .
$$

For instance, by (7.8)

$$
\left|(R(v), W)_{\dot{H}^{1}}\right|=\left|(R(v), \Delta W)_{L^{2}}\right| \leq\|R(v)\|_{L^{\frac{2 N}{N+2}}}\|\Delta W\|_{L^{2^{*}}}=O\left(\mathrm{~d}^{2}\right) .
$$

The formal integration by part in (7.10), which is rigorous for smooth solutions of (1.1) decaying fast enough at infinity, may be justified by passing to the limit and using the standard Cauchy problem theory for (1.1). Projecting equation (7.9) in $\dot{H}^{1}$ on $W, i W$ and $W_{1}$, we get (denoting by $\left.c:=\|W\|_{\dot{H}^{1}}^{2}, c_{1}:=\left\|W_{1}\right\|_{\dot{H}^{1}}^{2}\right)$

$$
\begin{gathered}
c \alpha_{s}=-\left(\Delta \tilde{u}_{2}, W\right)_{\dot{H}^{1}}-\left(W^{p_{c}-1} \tilde{u}_{2}, W\right)_{\dot{H}^{1}}+O(\varepsilon(s)) \\
c \theta_{s}=-\left(\Delta \tilde{u}_{1}, W\right)_{\dot{H}^{1}}-p_{c}\left(W^{p_{c}-1} \tilde{u}_{1}, W\right)_{\dot{H}^{1}}-\alpha\left(p_{c}-1\right)\left(W^{p_{c}}, W\right)_{\dot{H}^{1}}+O(\varepsilon(s)) \\
\frac{\mu_{s}}{\mu} c_{1}=-\left(\Delta \tilde{u}_{2}, W_{1}\right)_{\dot{H}^{1}}-\left(W^{p_{c}-1} \tilde{u}_{2}, W_{1}\right)_{\dot{H}^{1}}+O(\varepsilon(s)) .
\end{gathered}
$$

Justifying as before the integrations by parts, we have

$$
\left(\Delta \tilde{u}_{1}, W\right)_{\dot{H}^{1}}=\left(\tilde{u}_{1}, \Delta W\right)_{\dot{H}^{1}}, \quad\left(\Delta \tilde{u}_{2}, W\right)_{\dot{H}^{1}}=\left(\tilde{u}_{2}, \Delta W\right)_{\dot{H}^{1}}, \quad\left(\Delta \tilde{u}_{2}, W_{1}\right)_{\dot{H}^{1}}=\left(\tilde{u}_{2}, \Delta W_{1}\right)_{\dot{H}^{1}} .
$$

Consequently all the right-hand terms in equations (7.11), (7.12) and (7.13) are bounded up to a constant by $\|\tilde{u}\|_{\dot{H}^{1}}+\varepsilon$. By (3.22),$\|\tilde{u}\|_{\dot{H}^{1}} \leq C$ d which yields (7.7) and completes the proof of Lemma 3.7.

7.2. Spectral properties of the linearized operator. This part of the appendix is dedicated to the proof of Lemma 5.1, which is a variation of the classical proof (see Gri90] and the survey Sch06] for similar results).

7.2.1. Proof of the existence of the eigenfunctions. Note that $\overline{\mathcal{L}(v)}=-\mathcal{L}(\bar{v})$, so that if $e_{0}>0$ is an eigenvalue for $\mathcal{L}$ with eigenfunction $\mathcal{Y}_{+},-e_{0}$ is an eigenvalue of $\mathcal{L}$ with eigenfunction $\overline{\mathcal{Y}}_{+}$. Let us show the existence of $\mathcal{Y}_{+}$. Writing $\mathcal{Y}_{1}=\operatorname{Re} \mathcal{Y}_{+}, \mathcal{Y}_{2}=\operatorname{Im} \mathcal{Y}_{+}$, we must solve

$$
\left\{\begin{array}{c}
\left(\Delta+p_{c} W^{p_{c}-1}\right) \mathcal{Y}_{1}=-e_{0} \mathcal{Y}_{2} \\
\left(\Delta+W^{p_{c}-1}\right) \mathcal{Y}_{2}=e_{0} \mathcal{Y}_{1} .
\end{array}\right.
$$


Let $V:=W^{p_{c}-1}$. The operator $-\Delta-V$ on $L^{2}$ with domain $H^{2}$ is self-adjoint and nonnegative, thus it has a unique square root $(-\Delta-V)^{\frac{1}{2}}$ with domain $H^{1}$ (see [Wei80]). Assume that there exist $f_{1} \in H^{4}$ such that

$$
P f_{1}=-e_{0}^{2} f_{1} \text {, where } P:=(-\Delta-V)^{\frac{1}{2}}\left(-\Delta-p_{c} V\right)(-\Delta-V)^{\frac{1}{2}} .
$$

Then taking

$$
\mathcal{Y}_{1}:=(-\Delta-V)^{\frac{1}{2}} f_{1}, \quad \mathcal{Y}_{2}:=\frac{1}{e_{0}}\left(-\Delta-p_{c} V\right)(-\Delta-V)^{\frac{1}{2}} f_{1},
$$

would yield a solution of system (7.14), showing the existence of $\mathcal{Y}_{+}$and $\mathcal{Y}_{-}$.

The remainder of the proof is devoted to proving that the operator $P$ on $L^{2}$ with domain $H^{4}$ has a strictly negative eigenvalue. Note that

$$
P=(\Delta+V)^{2}-\left(p_{c}-1\right)(-\Delta-V)^{\frac{1}{2}} V(-\Delta-V)^{\frac{1}{2}}
$$

is a relatively compact, selfadjoint, perturbation of $\Delta^{2}$, so that its essential spectrum is $[0,+\infty)$ (see [Wei80]). Thus we only need to show the following claim.

\section{Claim 7.1.}

$$
\sigma_{-}(P):=\inf \left\{(P f, f)_{L^{2}}, f \in D(P),\|f\|_{L^{2}}=1\right\}<0 .
$$

Proof. Note that $(P f, f)_{L^{2}}=-\left(\left(\Delta+p_{c} V\right) F, F\right)_{L^{2}}$, where $F:=(-\Delta-V)^{\frac{1}{2}} f$. Thus it is sufficient to find $F$ such that

$$
\left(\left(\Delta+p_{c} V\right) F, F\right)_{L^{2}}>0, \text { and } \exists g \in H^{4}, F=(\Delta+V) g .
$$

We distinguish two cases. First assume that $N=3,4$, so that $W \notin L^{2}$. Let $W_{a}(x):=$ $\chi(x / a) W(x)$, where $\chi$ is a smooth, radial function such that $\chi(r)=1$ for $r \leq 1$ and $\chi(r)=0$ for $r \geq 2$. We first claim

$$
\exists a>0, \quad E_{a}:=\int\left(\Delta+p_{c} V\right) W_{a} W_{a}>0 .
$$

Recall that $\Delta W=-W^{p_{c}}$. Thus

$$
\left(\Delta+p_{c} V\right) W_{a}=\left(p_{c}-1\right) \chi(x / a) W^{p_{c}}+\frac{2}{a}(\nabla \chi)(x / a) \cdot \nabla W+\frac{1}{a^{2}}(\Delta \chi)(x / a) W .
$$

Hence

$$
\int\left(\Delta+p_{c} V\right) W_{a} W_{a}=\int \chi_{a}^{2}\left(p_{c}-1\right) W^{p_{c}+1}+\underbrace{\frac{2}{a} \int(\nabla \chi)(x / a) \cdot \nabla W W}_{(A)}+\underbrace{\frac{1}{a^{2}} \int(\Delta \chi)(x / a) W^{2}}_{(B)} .
$$

According to the explicit expression (1.2) of $W, W \leq C|x|^{-(N-2)}$ and $|\nabla W| \leq C|x|^{-(N-1)}$ at infinity, which gives $|(A)|+|(B)| \leq \frac{C}{a}$ if $N=3,|(A)|+|(B)| \leq \frac{C}{a^{2}}$ if $N=4$. Hence (7.17).

Let us fix $a$ such that (7.17) holds. Recall that $W$ is not in $L^{2}$. Thus $\Delta+V$ is a selfadjoint operator on $L^{2}$, with domain $H^{2}$, and without eigenfunction. In particular the orthogonal of its range $R(\Delta+V)$ is $\{0\}$, and thus $R(\Delta+V)$ is dense in $L^{2}$. Let $\varepsilon>0$, and consider $G_{\varepsilon} \in H^{2}$ such that

$$
\left\|(\Delta+V) G_{\varepsilon}-(\Delta+V-1) W_{a}\right\|_{L^{2}} \leq \varepsilon
$$


Taking $F_{\varepsilon}:=(\Delta+V-1)^{-1}(\Delta+V) G_{\varepsilon}$, we obtain $\left\|(\Delta+V-1)\left(F_{\varepsilon}-W_{a}\right)\right\|_{L^{2}} \leq \varepsilon$ which implies $\left\|F_{\varepsilon}-W_{a}\right\|_{H^{2}} \leq \varepsilon\left\|(\Delta+V-1)^{-1}\right\|_{L^{2} \rightarrow L^{2}}$. Hence for some constant $C_{0}$,

$$
\left|\int_{\mathbb{R}^{N}}\left(\Delta+p_{c} V\right) F_{\varepsilon} F_{\varepsilon}-\int_{\mathbb{R}^{N}}\left(\Delta+p_{c} V\right) W_{a} W_{a}\right| \leq C_{0} \varepsilon
$$

As a consequence of (7.17), we get (7.16) for $F=F_{\varepsilon}, \varepsilon=\frac{E_{a}}{2 C_{0}}$, which shows the claim in the case $N=3,4$.

Assume now that $N=5$, so that $W$ is in $L^{2}$ and more generally in all spaces $H^{s}\left(\mathbb{R}^{N}\right)$. In this case $(R(\Delta+V))^{\perp}=N(\Delta+V)=\operatorname{span}\{W\}$, and thus

$$
\overline{R(\Delta+V)}=\left\{f \in L^{2},(f, W)_{L^{2}}=0\right\} .
$$

Furthermore, $\Delta+p_{c} V$ is a self-adjoint compact perturbation of $\Delta$ and $\left(\left(\Delta+p_{c} V\right) W, W\right)_{L^{2}}>0$, which shows that $\Delta+p_{c} V$ has a positive eigenvalue. Let $Z$ be the eigenfunction for this eigenvalue. Recalling that $\left(\Delta+p_{c} V\right) W_{1}=0$ we get, for any real number $\alpha$

$$
\int_{\mathbb{R}^{N}}\left(\Delta+p_{c} V\right)\left(Z+\alpha W_{1}\right)\left(Z+\alpha W_{1}\right)=\int_{\mathbb{R}^{N}}\left(\Delta+p_{c} V\right) Z Z>0 .
$$

By explicit calculation, $\left(W_{1}, W\right)_{L^{2}} \neq 0$, so that we can chose the real number $\alpha$ to have $(Z+$ $\left.\alpha W_{1}, W\right)_{L^{2}}=0$. Hence

$$
\left((\Delta+V-1)\left(Z+\alpha W_{1}\right), W\right)_{L^{2}}=\left(Z+\alpha W_{1},(\Delta+V-1) W\right)_{L^{2}}=-\left(Z+\alpha W_{1}, W\right)_{L^{2}}=0 .
$$

By (7.18), we can chose, for any $\varepsilon>0$ a function $G_{\varepsilon}$ in $H^{2}$ such that

$$
\left\|(\Delta+V) G_{\varepsilon}-(\Delta+V-1)\left(Z+\alpha W_{1}\right)\right\|_{L^{2}}<\varepsilon .
$$

As in the preceding case, $F_{\varepsilon}=(\Delta+V-1)^{-1}(\Delta+V) G_{\varepsilon}$ satisfies (7.16) for small $\varepsilon>0$. Claim 7.1 is shown for $N=3,4,5$, which concludes the proof of the existence of the real eigenvalues of $e_{0}$ and $-e_{0}$.

7.2.2. Decay at infinity of the eigenfunctions. To conclude the proof of Lemma 5.1, it remains to show that $\mathcal{Y}_{ \pm} \in \mathcal{S}\left(\mathbb{R}^{N}\right)$. By a simple boot-strap argument, it is easy to see that the eigenfunctions $\mathcal{Y}_{+}$and $\mathcal{Y}_{-}$are $C^{\infty}$. It remains to show the decay at infinity of $\mathcal{Y}_{+}, \mathcal{Y}_{-}$and all their derivatives.

Recall that the eigenfunctions $\mathcal{Y}_{+}$and $\mathcal{Y}_{-}$are complex conjugates. According to system (7.14) on $\mathcal{Y}_{1}=\operatorname{Re} \mathcal{Y}_{+}$and $\mathcal{Y}_{2}=\operatorname{Im} \mathcal{Y}_{+}$, it suffices to show the decay result on $\mathcal{Y}_{1}$ only. Furthermore, by Sobolev embeddings, we only have to show that the following property holds for all $k$ and $s$

$$
\left(\mathcal{P}_{k, s}\right) \quad \forall \varphi \in C_{0}^{\infty}\left(\mathbb{R}^{N} \backslash\{0\}\right), \exists C, \forall R \geq 1,\left\|\varphi(x / R) \mathcal{Y}_{1}\right\|_{H^{s}} \leq \frac{C}{(1+R)^{k}} .
$$

Recall that $\mathcal{Y}_{1}=\sqrt{-\Delta-V} f_{1}$, with $f_{1} \in H^{4}$, so that $\left(\mathcal{P}_{0,3}\right)$ is satisfied. We will show that for $k \geq 0, s \geq 3,\left(\mathcal{P}_{k, s}\right)$ implies $\left(\mathcal{P}_{k+1, s+1}\right)$. Assume $\left(\mathcal{P}_{k, s}\right)$ and consider $\varphi$ and $\tilde{\varphi}$ in $C_{0}^{\infty}\left(\mathbb{R}^{N} \backslash\{0\}\right)$ such that $\tilde{\varphi}$ is 1 on the support of $\varphi$. Note that by (17.14)

$$
\left(\Delta^{2}+e_{0}^{2}\right) \mathcal{Y}_{1}=-V \Delta \mathcal{Y}_{1}-\Delta\left(p_{c} V \mathcal{Y}_{1}\right)-p_{c} V^{2} \mathcal{Y}_{1}
$$

By the explicit form of $W, V$ and all its derivatives decay at least as $\frac{1}{|x|^{4}}$ at infinity. Thus (17.19) implies $\left\|\varphi(x / R)\left(\Delta^{2}+e_{0}^{2}\right) \mathcal{Y}_{1}\right\|_{H^{s-3}} \leq \frac{C}{R^{4}}\left\|\widetilde{\varphi}(x / R) \mathcal{Y}_{1}\right\|_{H^{s}}$. Hence

$$
\left\|\left(\Delta^{2}+e_{0}^{2}\right)\left(\varphi(x / R) \mathcal{Y}_{1}\right)\right\|_{H^{s-3}} \leq \frac{C}{R}\left\|\widetilde{\varphi}(x / R) \mathcal{Y}_{1}\right\|_{H^{s}}
$$


By $\left(\mathcal{P}_{k, s}\right)$, the right-hand side of (17.20) is bounded by $\frac{C}{R^{k+1}}$ for large $R$. Furthermore, $\Delta^{2}+e_{0}^{2}$ is an isomorphism from $H^{s+1}$ to $H^{s-3}$, so that (7.20) implies $\left\|\varphi(x / R) \mathcal{Y}_{1}\right\|_{H^{s+1}} \leq \frac{C}{R^{k+1}}$, which yields exactly $\left(\mathcal{P}_{k+1, s+1}\right)$. The proof is complete.

Remark 7.2. Let $\Psi \in \mathcal{S}\left(\mathbb{R}^{N}\right)$ and $e_{1} \in \mathbb{R} \backslash\left\{-e_{0}, 0, e_{0}\right\}$ (thus by Corollary [5.3, $e_{1}$ is not in the spectrum of $\mathcal{L}$ ). Then by a proof similar to the one above

$$
\Phi:=\left(\mathcal{L}-e_{1}\right)^{-1} \Psi \in \mathcal{S}\left(\mathbb{R}^{N}\right) .
$$

Indeed $\Phi_{1}=\operatorname{Re} \Phi$ and $\Phi_{2}=\operatorname{Im} \Phi$ satisfy the equations

$$
-e_{1} \Phi_{1}+(\Delta+V) \Phi_{2}=\Psi_{1}, \quad-e_{1} \Phi_{2}-\left(\Delta+p_{c} V\right) \Phi_{1}=\Psi_{2}
$$

As $\Phi_{1}$ and $\Phi_{2}$ are, by definition, in $L^{2}$, a simple bootstrap argument shows that they are in all $H^{s}, s \geq 0$. Furthermore

$$
\left(\Delta^{2}+e_{1}^{2}\right) \Phi_{1}=-V \Delta \Phi_{1}-\Delta\left(p_{c} V \Phi_{1}\right)-p_{c} V^{2} \Phi_{1}-e_{1} \Psi_{1}-(\Delta+V) \Psi_{2},
$$

which gives equation (7.19), up to a right-member term $-e_{1} \Psi_{1}-(\Delta+V) \Psi_{2}$ which is in $\mathcal{S}\left(\mathbb{R}^{N}\right)$. Thus the iteration argument above shows that $\Phi_{1} \in \mathcal{S}\left(\mathbb{R}^{N}\right)$, which implies by (7.22) that $\Phi_{2} \in$ $\mathcal{S}\left(\mathbb{R}^{N}\right)$. Hence (7.21).

\subsection{Proof of Lemma 5.6. We have}

$$
R(f)=-i|W+f|^{p_{c}-1}(W+f)+i W^{p_{c}}+i \frac{p_{c}+1}{2} W^{p_{c}-1} f+i \frac{p_{c}-1}{2} W^{p_{c}-1} \bar{f}=W^{p_{c}} J\left(W^{-1} f\right)
$$

where $J$ is the function defined on $\mathbb{C}$ by

$$
J(z)=-i|1+z|^{p_{c}-1}(1+z)+i+i \frac{p_{c}+1}{2} z+i \frac{p_{c}-1}{2} \bar{z} .
$$

Recall that $p_{c}>2$. Thus $J$ is of class $C^{2}$ on $\mathbb{C}$ and $J(0)=\partial_{z} J(0)=\partial_{\bar{z}} J(0)=0$. Furthermore, for large $|z|, J$ is bounded by $C|z|^{p_{c}}$, and its derivatives of order $k=1,2$ by $C|z|^{p_{c}-k}$. Hence

$$
\begin{aligned}
\left|J(z)-J\left(z^{\prime}\right)\right| & \leq C\left|z-z^{\prime}\right|\left(|z|+\left|z^{\prime}\right|+|z|^{p_{c}-1}+\left|z^{\prime}\right|^{p_{c}-1}\right) \\
\left|\partial_{z} J(z)-\partial_{z} J\left(z^{\prime}\right)\right|+\left|\partial_{\bar{z}} J(z)-\partial_{\bar{z}} J\left(z^{\prime}\right)\right| & \leq C\left|z-z^{\prime}\right|\left(1+|z|^{p_{c}-2}+\left|z^{\prime}\right|^{p_{c}-2}\right) .
\end{aligned}
$$

By (7.23) we get the pointwise bound

$$
|R(f)-R(g)| \leq C|f-g|\left(W^{p_{c}-2}|f|+W^{p_{c}-2}|g|+|f|^{p_{c}-1}+|g|^{p_{c}-1}\right),
$$

which yields (5.27) using Hölder inequality $\left\|a b c^{p_{c}-2}\right\|_{L^{\frac{2 N}{N+2}}} \leq\|a\|_{L^{2^{*}}}\|b\|_{L^{2^{*}}}\|c\|_{L^{2^{*}}}^{p_{c^{*}}-2}$.

Now, remark that

$$
\begin{aligned}
\nabla(R(f))=p_{c} W^{p_{c}-1}(\nabla W) J & \left(W^{-1} f\right) \\
& +W^{p_{c}} \nabla\left(W^{-1} f\right)\left(\partial_{z} J\right)\left(W^{-1} f\right)+W^{p_{c}} \nabla\left(W^{-1} f\right)\left(\partial_{\bar{z}} J\right)\left(W^{-1} f\right) .
\end{aligned}
$$


By (7.23) and (7.24) we get

$$
\begin{gathered}
|\nabla R(f)-\nabla R(g)| \leq C\{(A)+(B)+(C)\} \\
(A):=\frac{1}{|x|+1}|f-g|\left(W^{p_{c}-2}|f|+W^{p_{c}-2}|g|+|f|^{p_{c}-1}+|g|^{p_{c}-1}\right) \\
(B):=\left|W \nabla\left(W^{-1} f-W^{-1} g\right)\right|\left(W^{p_{c}-2}|f|+|f|^{p_{c}-1}\right) \\
(C):=\left|W \nabla\left(W^{p_{c}-2}+W^{-1} g\right)\right||f-g|\left(W^{p_{c}-2}+|f|^{p_{c}-2}+|g|^{p_{c}-2}\right) .
\end{gathered}
$$

Note that $\frac{2 N(N+2)}{N^{2}+4}<N$ for $N=3,4,5$ so that if $u \in S(I)$ and $\nabla u \in Z(I)$, Hardy inequality $\left\|\frac{1}{|x|} u\right\|_{Z(I)} \leq\|\nabla u\|_{Z(I)}$ holds. Using Hölder inequality $\left\|a b c^{p_{c}-2}\right\|_{N(I)} \leq\|a\|_{Z(I)}\|b\|_{S(I)}\|c\|_{S(I)}^{p_{c}-2}$ together with Hardy and Sobolev inequalities we get

$$
\begin{aligned}
\|(A)\|_{N(I)} & \leq C\left\|\frac{1}{|x|+1}(f-g)\right\|_{Z(I)}\left[\|W\|_{S(I)}^{p_{c}-2}\left(\|f\|_{S(I)}+\|g\|_{S(I)}\right)+\|f\|_{S(I)}^{p_{c}-1}+\|g\|_{S(I)}^{p_{c}-1}\right] \\
& \leq C\|\nabla(f-g)\|_{Z(I)}\left[|I|^{\frac{6-N}{2(N+2)}}\left(\|\nabla f\|_{Z(I)}+\|\nabla g\|_{Z(I)}\right)+\|\nabla f\|_{Z(I)}^{p_{c}-1}+\|\nabla g\|_{Z(I)}^{p_{c}-1}\right] .
\end{aligned}
$$

The other terms $(B)$ and $(C)$ are handled in the same way. Note in particular that by Hardy inequality $\left\|W \nabla\left(W^{-1} g\right)\right\|_{Z(I)} \leq C\|\nabla g\|_{Z(I)}$. The proof of (5.28) is complete.

\section{REFERENCES}

[Aub76] Thierry Aubin. Équations différentielles non linéaires et problème de Yamabe concernant la courbure scalaire. J. Math. Pures Appl. (9), 55(3):269-296, 1976.

[Bou99a] J. Bourgain. Global solutions of nonlinear Schrödinger equations, volume 46 of American Mathematical Society Colloquium Publications. American Mathematical Society, Providence, RI, 1999.

[Bou99b] J. Bourgain. Global wellposedness of defocusing critical nonlinear Schrödinger equation in the radial case. J. Amer. Math. Soc., 12(1):145-171, 1999.

$\left[\mathrm{CMS}^{+}\right.$06] J. Colliander, Keel M., G. Staffilani, H. Takaoka, and T. Tao. Global well-posedness and scattering for the critical non-linear schrödinger equation in $\mathbf{R}^{3}$. To appear in Annals of Mathematics, 2006.

[CW90] Thierry Cazenave and Fred B. Weissler. The Cauchy problem for the critical nonlinear Schrödinger equation in $H^{s}$. Nonlinear Anal., 14(10):807-836, 1990.

[Gri90] Manoussos Grillakis. Analysis of the linearization around a critical point of an infinite-dimensional Hamiltonian system. Comm. Pure Appl. Math., 43(3):299-333, 1990.

[GV85] J. Ginibre and G. Velo. The global Cauchy problem for the nonlinear Schrödinger equation revisited. Ann. Inst. H. Poincaré Anal. Non Linéaire, 2(4):309-327, 1985.

[Ker01] Sahbi Keraani. On the defect of compactness for the Strichartz estimates of the Schrödinger equations. J. Differential Equations, 175(2):353-392, 2001.

[KM06] Carlos E. Kenig and Frank Merle. Global well-posedness, scattering and blow-up for the energy-critical, focusing, non-linear schrödinger equation in the radial case. Preprint, 2006.

[KT98] Markus Keel and Terence Tao. Endpoint Strichartz estimates. Amer. J. Math., 120(5):955-980, 1998.

[Lio85] P.-L. Lions. The concentration-compactness principle in the calculus of variations. The limit case. II. Rev. Mat. Iberoamericana, 1(2):45-121, 1985.

[Mer93] Frank Merle. Determination of blow-up solutions with minimal mass for nonlinear Schrödinger equations with critical power. Duke Math. J., 69(2):427-454, 1993.

[Rey90] Olivier Rey. The role of the Green's function in a nonlinear elliptic equation involving the critical Sobolev exponent. J. Funct. Anal., 89(1):1-52, 1990.

[Sch06] Wilhelm Schlag. Spectral theory and nonlinear partial differential equations: a survey. Discrete Contin. Dyn. Syst., 15(3):703-723, 2006.

[SK05] W. Schlag and J. Krieger. On the focusing critical semi-linear wave equation. To appear in American Journal of Mathematics, 2005. 
[Str77a] Walter A. Strauss. Existence of solitary waves in higher dimensions. Comm. Math. Phys., 55(2):149$162,1977$.

[Str77b] Robert S. Strichartz. Restrictions of Fourier transforms to quadratic surfaces and decay of solutions of wave equations. Duke Math. J., 44(3):705-714, 1977.

[Tal76] Giorgio Talenti. Best constant in Sobolev inequality. Ann. Mat. Pura Appl. (4), 110:353-372, 1976.

[TV05] Terence Tao and Monica Visan. Stability of energy-critical nonlinear Schrödinger equations in high dimensions. Electron. J. Differential Equations, pages No. 118, 28 pp. (electronic), 2005.

[Wei80] Joachim Weidmann. Linear operators in Hilbert spaces, volume 68 of Graduate Texts in Mathematics. Springer-Verlag, New York, 1980. Translated from the German by Joseph Szücs.

E-mail address: thomas.duyckaerts@u-cergy.fr

Thomas Duyckaerts, Université de Cergy-Pontoise, Département de Mathématiques, Site de Saint Martin, 2 avenue Adolphe-Chauvin, 95302 Cergy-Pontoise cedex, France. 